\title{
Robust Encoding of a Qubit in a Molecule
}

\author{
Victor V. Albert๑, ${ }^{1,2}$ Jacob P. Covey®, ${ }^{1}$ and John Preskill $\oplus^{1,2}$ \\ ${ }^{1}$ Institute for Quantum Information and Matter, California Institute of Technology, \\ Pasadena, California 91125, USA \\ ${ }^{2}$ Walter Burke Institute for Theoretical Physics, California Institute of Technology, \\ Pasadena, California 91125, USA
}

(Received 18 November 2019; revised 27 May 2020; accepted 21 July 2020; published 1 September 2020)

\begin{abstract}
We construct quantum error-correcting codes that embed a finite-dimensional code space in the infinitedimensional Hilbert space of rotational states of a rigid body. These codes, which protect against both drift in the body's orientation and small changes in its angular momentum, may be well suited for robust storage and coherent processing of quantum information using rotational states of a polyatomic molecule. Extensions of such codes to rigid bodies with a symmetry axis are compatible with rotational states of diatomic molecules as well as nuclear states of molecules and atoms. We also describe codes associated with general non-Abelian groups and develop orthogonality relations for coset spaces, laying the groundwork for quantum information processing with exotic configuration spaces.
\end{abstract}

DOI: $10.1103 /$ PhysRevX.10.031050

\section{INTRODUCTION}

Quantum systems described by continuous variables arise in many laboratory settings. For example, a microwave resonator in a superconducting circuit or the motional degree of freedom of a trapped ion can be viewed as a harmonic oscillator with an infinite-dimensional Hilbert space. Such continuous-variable systems have potential applications to quantum information processing. However, quantum information encoded in an oscillator can be easily damaged by ubiquitous noise sources such as dissipation and diffusive motion in phase space.

Robustness against noise can be achieved more easily by encoding a protected finite-dimensional system within the infinite-dimensional Hilbert space of an oscillator. One method for doing so was proposed some years ago by Gottesman, Kitaev, and Preskill (GKP) [1]. A GKP code is a quantum error-correcting code designed to protect against noise that slightly shifts the position or momentum of an oscillator. The ideal basis states for the code space are "grid states" supported on periodically spaced points in position or momentum space. By measuring the code's check operators, one can diagnose a shift error that may have occurred, without disturbing the encoded quantum

Published by the American Physical Society under the terms of the Creative Commons Attribution 4.0 International license. Further distribution of this work must maintain attribution to the author(s) and the published article's title, journal citation, and DOI.
Subject Areas: Atomic and Molecular Physics,

Chemical Physics,

Quantum Information (a) Planar rotor $\mathrm{U}_{1}$

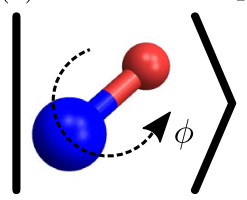

(b) Rigid rotor $\mathrm{SO}_{3}$

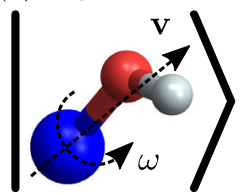

(c) Linear rotor $\mathrm{S}^{2}$
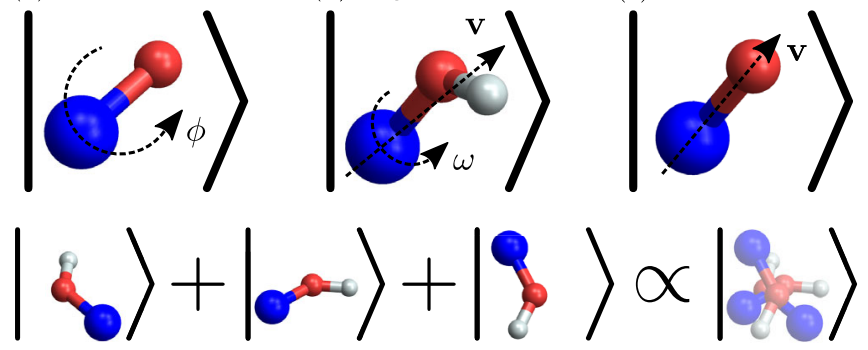

$\mid 0,0=0+1$
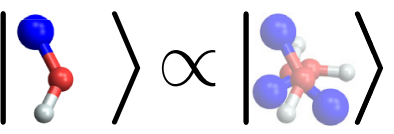

FIG. 1. Rigid bodies. A molecular code protects against errors in the orientation and angular momentum of a rigid body, which may be (a) a planar rotor whose orientation is an element of the two-dimensional rotation group $\mathrm{U}_{1}$, (b) a rigid rotor whose orientation is an element of the three-dimensional rotation group $\mathrm{SO}_{3}$, or (c) a linear rotor whose orientation is a point on the twosphere $S^{2}$. A basis state for the code, or code word, is a superposition of a finite number of orientations.

information, and then correct the error (if the shift introduced by noise is not too large) by performing a compensating shift. These codes are expected to perform well against realistic noise, including dissipation, which typically acts locally in phase space [2-4]. Construction of GKP grid states has recently been demonstrated experimentally $[5,6]$.

In this paper, we develop GKP-like codes that protect against, not noise that shifts the position and momentum of an oscillator, but rather noise that shifts the (continuous) orientation and (discrete) angular momentum of an asymmetric rigid body. GKP codes for objects that rotate 
about a fixed axis [Fig. 1(a)] were already discussed in Ref. [1]. In that case, the orientation of the object corresponds to an element of the two-dimensional rotation group $\mathrm{U}_{1}=\mathrm{SO}_{2}=\mathrm{C}_{\infty}$. New issues arise for an object that rotates freely in three dimensions [Figs. 1(b) and 1(c)], with orientation described as an element of the three-dimensional rotation group $\mathrm{SO}_{3}$ (for an object with no symmetries) or a point on the two-sphere $\mathrm{S}^{2}=\mathrm{SO}_{3} / \mathrm{U}_{1}$ (for an object with a symmetry axis).

Our work is motivated by recent progress in trapping and coherently manipulating individual diatomic and polyatomic molecules [7-16]. Molecules offer long coherence times in both their nuclear and rotational states, have builtin long-range dipolar interactions, and can be scaled up to large arrays without compromising on their indistinguishability, coherence time, or interaction fidelity. Furthermore, couplings between a molecule's internal degrees of freedom can be readily engineered and utilized. These features beg the question of whether it is possible to utilize the rich yet spatially compact molecular Hilbert space for quantum error correction; our work shows that such is indeed the case. Since we consider only a molecule's rotational degrees of freedom, for our purposes a molecule is equivalent to a rigid body. For that reason, we refer to quantum codes embedding a protected finite-dimensional subspace in the infinite-dimensional Hilbert space of a rigid body as molecular codes.

The rigid-rotor Hamiltonian describing molecular rotational motion is inherently anharmonic; because the energy levels are unevenly spaced, transitions between levels can be individually addressed using microwave fields. Hence, proposals for storing quantum information in molecules [17-28] (see also Refs. [29,30]) typically pick out two lowlying long-lived energy eigenstates as basis states for a qubit. One can also introduce an external electric field and encode a qubit using the resulting "pendular" eigenstates [31-33]. Other proposals advocate using vibrational or spin degrees of freedom [34,35].

Rigid-rotor energy eigenstates, if spaced sufficiently far apart in angular momentum, provide protection against small jumps in angular momentum, but are unprotected against dephasing in the angular-momentum eigenstate basis resulting from fluctuations in the rotor's orientation. Our molecular codes, inspired by GKP codes, are designed to protect against both momentum kicks and orientational diffusion of a single molecule. Here, we develop the theory of molecular codes and generalizations thereof. Laboratory realizations of these coding schemes that actually improve the coherence times of molecular qubits may still be far off, but we propose laying the foundations for molecular quantum error correction as a challenging goal for the physicists and chemists of the noisy intermediate-scale quantum (NISQ) era [36].

Though our work is partially motivated by advances in molecular physics, the coding methods we use are best explained in an abstract group-theoretic framework, which we summarize in the next section. In Sec. III, we enumerate a variety of physical settings, in molecular physics and beyond, where our code constructions may be applicable. The connection with rotational states of the three molecular rotors from Figs. 1(a)-1(c) is developed in greater detail in Secs. IV-VI, respectively. Section VII discusses extensions to more abstract state spaces. Section VIII contains conclusions and ideas for future work.

\section{SUMMARY OF OUR FRAMEWORK}

We describe a family of codes that generalize the GKP codes [1], which were initially formulated to encode a finite-dimensional system in the infinite-dimensional Hilbert space of a bosonic mode or of many bosonic modes. Each code in our generalized GKP code family is associated with a nested sequence of groups

$$
\mathrm{H} \subset \mathrm{K} \subset \mathrm{G} \text {. }
$$

Here, $\mathrm{G}$ is a continuous group of shifts in the position of a physical object. If no nontrivial subgroup of $G$ leaves the object invariant, and any position can be reached by applying an element of $G$ to a standard initial position, then we may regard the "position eigenstates" $\{|g\rangle, g \in \mathrm{G}\}$ as a basis for the Hilbert space of the object. The generalized GKP code is a subspace of this Hilbert space defined by two properties: (i) The discrete subgroup $\mathrm{H}$ of the continuous group $\mathrm{G}$ leaves any state in the code space invariant, and (ii) the subgroup $\mathrm{K}$ acts transitively on a basis for the code space.

For the standard GKP code, $\mathrm{G}$ is the Abelian noncompact group $\mathrm{R}$, the group of translations in position space of a particle in one spatial dimension. The subgroup $\mathrm{K}$ is the infinite discrete group containing all translations of the particle by an integer multiple of $\alpha$, where $\alpha$ is a fixed real number. The subgroup $\mathrm{H}$ contains all translations by an integer multiple of $d \alpha$, where $d$ is the dimension of the code space. In this case, we may choose the basis for the code space to be (up to normalization)

$$
|\bar{k}\rangle \propto \sum_{h \in Z}|q=(k+h d) \alpha\rangle,
$$

where $|q\rangle$ is a position state of the oscillator and $k \in\{0,1, \ldots, d-1\}$. We refer to each such basis element of the code as a code word. Thus, a translation of $q$ by $d \alpha$ leaves the code words invariant, and a translation of $q$ by $\alpha$ permutes the code words according to $k \rightarrow k+1$ modulo $d$. A shift in $q$ due to an error can be detected by measuring $q$ modulo $\alpha$.

In addition to errors that shift the value of $q$, the GKP code also protects against errors that introduce $q$-dependent phases. Phase errors which are diagonal in the $q$ basis are described by functions on $\mathrm{R}$. Such functions can be 
Fourier-expanded using irreducible representations (irreps) of $\mathrm{R}$, labeled by the momentum $p$. The irreps that preserve the code space are those with $p$ an integer multiple of $2 \pi / d \alpha$, and those that act trivially on the code space have $p$ an integer multiple of $2 \pi / \alpha$.

For a generalized GKP code, the detectable position shifts are labeled by elements of the coset space $\mathrm{G} / \mathrm{K}$, and the "logical" position shift errors that preserve the code space are labeled by elements of $\mathrm{K} / \mathrm{H}$. Undetectable logical phase errors correspond to representations of $\mathrm{G}$ which represent the subgroup $\mathrm{H}$ trivially but represent $\mathrm{K}$ nontrivially.

In Sec. IV, we illustrate the concepts underlying generalized GKP codes by discussing the example of a planar rotor. In this case, $\mathrm{G}$ is $\mathrm{U}_{1}$, the infinite compact group of rotations in a two-dimensional plane, $\mathrm{K}$ is the finite subgroup of $\mathrm{U}_{1}$ containing rotations by an angle which is an integer multiple of $2 \pi / d N$, and $\mathrm{H}$ is the subgroup of $\mathrm{K}$ containing rotations by an angle which is an integer multiple of $2 \pi / N$. Here, $N$ and $d$ are positive integers, and $d$ is the dimension of the code space. This code can correct a rotation of the planar rotor by any angle less than $\pi / d N$ and can correct a shift in angular momentum by any integer less than $N / 2$. The structure of this code, for the case $N=3$ and $d=2$, is depicted in Fig. 2(a).

While these planar-rotor codes were already introduced in Ref. [1], generalized GKP codes where $\mathrm{G}$ is non-Abelian have not been previously discussed to our knowledge. In Sec. V, we introduce molecular codes, which can protect an asymmetric rigid body from rotational shift errors and angular-momentum kicks. In this case, $\mathrm{G}$ is $\mathrm{SO}_{3}$, the infinite compact group of proper rotations in 3D space. The finite subgroups $\mathrm{H} \subset \mathrm{K} \subset \mathrm{SO}_{3}$ can be chosen in various ways. By choosing $\mathrm{H}=\mathrm{Z}_{N} \subset \mathrm{K}=\mathrm{Z}_{d N}$ to be discrete cyclic groups of rotations about one axis (for chemists, $\mathrm{Z}_{N}=\mathrm{C}_{N}$ ), we obtain codes that can correct small rotations of the body about any axis and can also correct momentum kicks that change the total angular momentum of the body by $\delta \ell<N / 2$. For a pictorial representation of this code in the case $N=3, d=2$, see Fig. 2(b). Each ideal code word is not normalizable, a super-position of a finite number of position eigenstates, but there are normalizable approximate code words which maintain good error-correcting properties. We also discuss examples where $H$ and $K$ are finite non-Abelian subgroups of the rotation group.

We generalize the code construction further in Sec. VII, where we allow $\mathrm{G}$ to be any finite group, compact Lie group, or sufficiently well-behaved noncompact group. Guided by the stabilizer formalism, we show that for each molecular code there is a Hamiltonian which has the code as its ground space. This formulation provides a unified treatment that encompasses molecular codes $\left(\mathrm{G}=\mathrm{SO}_{3}\right)$, Calderbank-Shor-Steane (CSS) codes $\left(\mathrm{G}=\mathrm{Z}_{D}^{\times n}\right)$, and GKP codes for qudits $\left(\mathrm{Z}_{D}\right)$, planar rotors $\left(\mathrm{U}_{1}\right.$ or $\left.\mathrm{Z}\right)$, and oscillators (R).

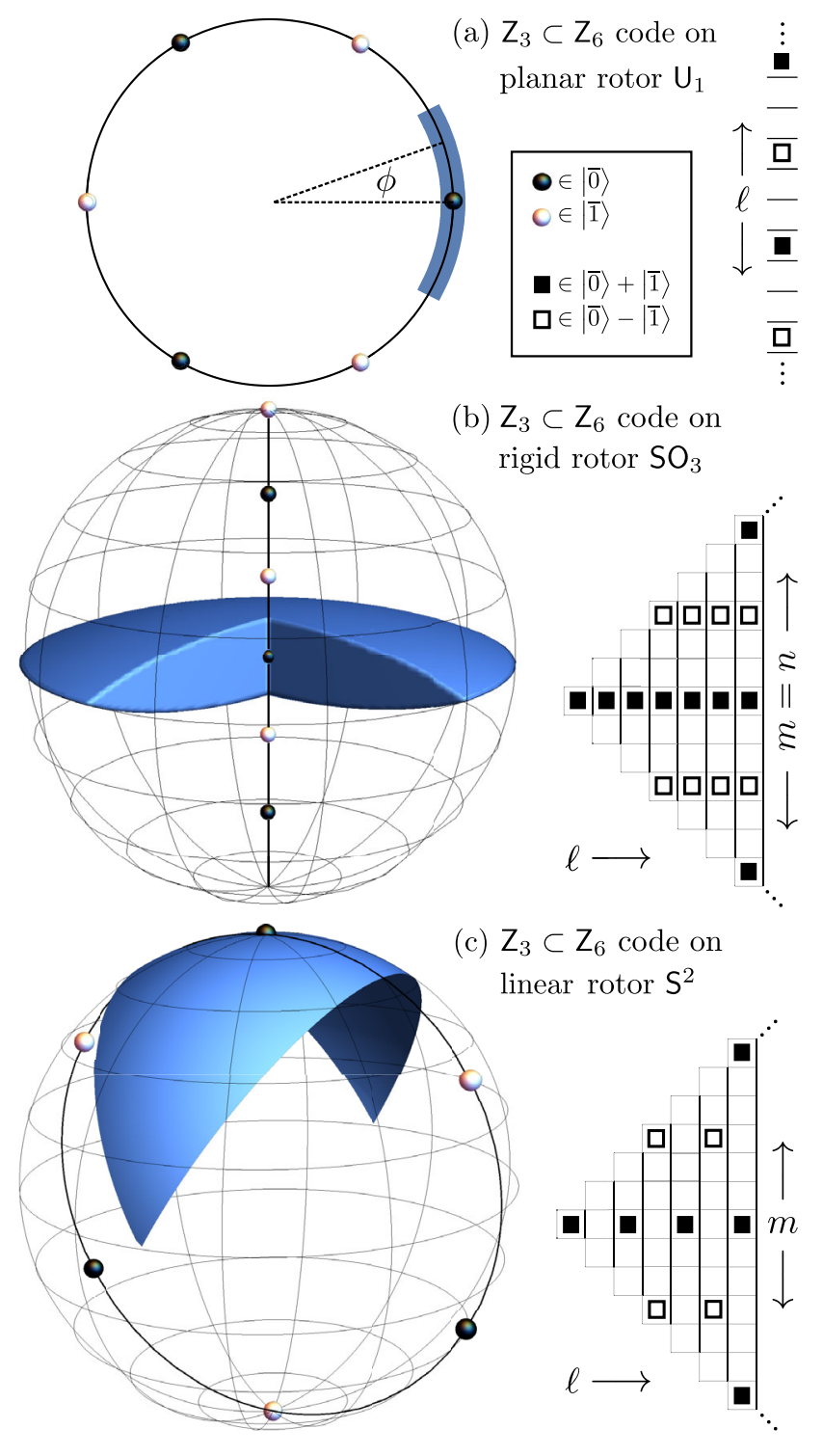

FIG. 2. Code word constructions. (a) Left: Sketch of the planarrotor state space $U_{1}$. Black and white points represent the positions present in the two code words (10) of the $\mathrm{Z}_{3} \subset \mathrm{Z}_{6}$ GKP rotor code. Correctable shifts $(-\pi / 6, \pi / 6]$ are highlighted in blue. Right: $U_{1}$ angular-momentum ladder $\ell \in \mathrm{Z}$. Black and white squares represent momentum states present in the logical- $X$ code words (11). (b) Sketch of the same features of the $\mathrm{Z}_{3} \subset \mathrm{Z}_{6}$ molecular code (45). Left: Position space is drawn as a ball of radius $\pi$ with antipodal points identified, and each $\mathrm{SO}_{3}$ rotation by angle $\omega$ around axis $\mathbf{v} \in \mathrm{S}^{2}$ corresponds to the vector $\omega \mathbf{v}$ on the ball. The set of correctable rotations is in blue, but part of it is cut out to show that it contains the origin (meaning that small rotations around any axis are correctable). Right: Momentum space is a 3D square pyramid with the height labeled by $\ell$ and the base by $|m|,|n| \leq \ell$. We plot only the $m=n$ part, where the code words (49) have support. (c) Sketch of similar features of the $\mathrm{Z}_{3} \subset \mathrm{Z}_{6}$ linear-rotor code (102), whose states are equal superpositions of equidistant orientations along an equator. Left: The blue spherical lune contains all points that are closer to the enclosed black point than to any other black or white point. Right: Momentum space is a $2 \mathrm{D}$ pyramid with base $|m| \leq \ell$, showing states participating in the logical- $X$ code words (105). 
In Sec. VI, we discuss the linear rotor, a rigid body with a symmetry axis, such as a heteronuclear diatomic molecule. For this case, the quantum codes we construct are not generalized GKP codes as defined above, because the position basis states of the linear rotor are indexed not by elements of a group but rather by points in the coset space $\mathrm{SO}_{3} / \mathrm{U}_{1}=\mathrm{S}^{2}$. Code words of a linear-rotor code are uniform superpositions of antipodal points on $\mathrm{S}^{2}$, which lie in the same orbit of $\mathrm{H}$ acting on $\mathrm{S}^{2}$, where $\mathrm{H}$ is a finite subgroup of $\mathrm{SO}_{3}$. See Fig. 2(c) for the case $\mathrm{H}=\mathrm{Z}_{3}$ and code space dimension two.

The linear-rotor codes can also correct small rotations about any axis, and analyzing correction of momentum kicks follows closely the corresponding discussion for molecular codes. However, for correction of combinations of rotations and momentum kicks, there are complications which arise, because each $\mathrm{SO}_{3}$ rotation acting on $\mathrm{S}^{2}$ has fixed points.

Coset spaces arise in both generalized GKP codes and linear-rotor codes, but for different reasons. In GKP codes, position basis states are in one-to-one correspondence with elements of the group $\mathrm{G}$, and the position shifts detected by the code are labeled by elements of $\mathrm{G} / \mathrm{K}$. In linear-rotor codes, the position basis states themselves are in one-to-one correspondence with elements of the coset space $\mathrm{SO}_{3} / \mathrm{U}_{1}$. Since coset spaces play a central role in both settings, we formulate position and momentum bases, shift operators, and orthogonality relations for general $\mathrm{G} / \mathrm{H}$ in the Appendix D. These are applicable to H-symmetric molecules when $\mathrm{G}=\mathrm{SO}_{3}$ (see Sec. IIIC) and may be of independent interest for general G.

\section{EXPERIMENTAL REALIZATIONS}

Before proceeding to discuss code constructions in more detail, in this section, we briefly mention some of the physical settings where these constructions might apply. The rotational states of a molecule provide one such setting, where the orientations of a molecule correspond to elements of $\mathrm{SO}_{3}$ (in the case of an asymmetric polyatomic molecule) or $\mathrm{S}^{2}$ (in the case of a heteronuclear diatomic molecule). In addition, other physical systems, including atomic or molecular hyperfine, vibrational, and electronic states, as well as atomic ensembles and levitated nanoparticles, realize similar configuration spaces.

\section{A. Molecular rotors}

GKP codes were realized experimentally $[5,6]$ nearly 20 years after the initial proposal [1], and full-fledged error correction for molecular qubits may still be many years away (see Ref. [37], Sec. VD). Nevertheless, significant steps toward the realization of molecular codes may be feasible during the NISQ era [36] as the technology for trapping and controlling molecules [38-43] continues to advance.

Laser cooling and trapping techniques have recently enabled several seminal advances for diatomic polar molecules, namely, the creation of low-entropy arrays in an optical lattice $[44,45]$, trapping and imaging in tweezer arrays $[10,12]$ and magnetic traps $[46,47]$, preparation of pure quantum states [48], and the first quantum degenerate gas of polar molecules [49]. Recent efforts have succeeded in controlling rotational states of $\mathrm{CaH}^{+}$[15] as well as coupling them to a neighboring ion [16]. Coherence times of approximately $100 \mathrm{~ms}$ to approximately $1 \mathrm{~s}$ in angularmomentum states of diatomic polar molecules have already been observed in several experiments [50-52].

Laser cooling and quantum control of polyatomic molecules continues to be a rapidly progressing field $[11,37,53,54]$. The possibility of angular-momentum state-resolved detection has recently been considered $[13,37]$. In addition, quantum gates of optically trapped symmetric top molecules have recently been analyzed [26]. Symmetric top molecules also hold promise for simulating quantum magnetism $[55,56]$. Moreover, specific classes of polyatomic linear polar molecules that feature more than one optically active metal atom have recently been proposed for laser cooling and trapping [57]. Prospects for cooling other complex polyatomic molecules have also been analyzed $[58,59]$.

Here, we highlight a few techniques that could help realize aspects of our codes in real systems.

\section{Rotational states}

The code words for our codes can be expressed as coherent superpositions of several different molecular orientations. Alternatively, each code word can be expressed as a coherent superposition of eigenstates of angular momentum (also known as "rotational states"). When discussing experimental realization of the codes, the basis of rotational states is far more convenient than the position-eigenstate basis, because rotational states can be directly addressed using experimental tools.

For the case of a planar rotor, with configuration space $\mathrm{U}_{1}$, the rotational basis states $\{|\ell\rangle\}$ transform as onedimensional irreducible representations of $\mathrm{U}_{1}$; for the case of a polyatomic molecule, with configuration space $\mathrm{SO}_{3}$, the basis states $\left.\left\{||_{m n}^{\ell}\right\rangle\right\}$ correspond to matrix elements of irreducible representations of $\mathrm{SO}_{3}$; and for the case of a diatomic molecule, with configuration space $S^{2}$, the basis states $\left.\left\{||_{m}^{\ell}\right\rangle\right\}$ correspond to spherical harmonics. In molecular physics [60-63], $\ell$ corresponds to the total angular momentum of the rotor, $m$ is the $\mathbf{z}$ component of the angular momentum in the lab frame, and $n$ is the $\mathbf{z}$ component in the rotor frame. How code words are expressed as linear combinations of rotational states is illustrated on the right in Figs. 2(a)-2(c), respectively, for three simple rotor codes.

\section{Microwave dressing}

One can try to stabilize the code words using polychromatic microwave dressing. Note that we consider 
single molecules and neglect any effects due to their interaction. The inherent rigid-rotor Hamiltonian for $\mathrm{SO}_{3}$ and $\mathrm{S}^{2}$ is diagonal in the angular-momentum basis, with eigenvalues $\ell(\ell+1)$ [64]. Therefore, transitions between states with different momenta are individually addressable (unlike, e.g., the transitions of a harmonic oscillator Hamiltonian). Selection rules for the internal indices $n$, $m$ are dictated by the polarization of the microwave field. Thus, the energy and polarization of a microwave field can be tuned to couple two angular-momentum states that are neighbors in the angular-momentum pyramid. That is, the value of $\ell, m$, or $n$ can change by one unit in a singlephoton transition.

However, the angular-momentum states making up each code word are widely spaced in the internal indices. For example, in the case of the $\mathrm{SO}_{3}$ code depicted in Fig. 2(b), the code words have support only on $\left.\left\{||_{m n}^{\ell}\right\rangle\right\}$ states such that $m=n$ is an integer multiple of 3 . For $S^{2}$ [Fig. 2(c)], a similar pattern emerges, except that, for even $\ell$, the rotational state $\left|\begin{array}{l}\ell \\ m\end{array}\right\rangle$ is populated only if $m$ is an even multiple of 3 , while, for odd $\left.\ell,\left\{||_{m}^{\ell}\right\rangle\right\}$ is populated only if $m$ is an odd multiple of 3.

Because a single microwave tone couples states that differ by just one unit of $m$ or $\ell$, a sequence of virtual transitions induced by multiple pulses would be needed to couple states with more widely separated values of $m$ or $\ell$. For example, coupling states with $|\delta m|=3$ requires a three-photon transition that is sufficiently detuned from the two intermediate states, and coupling states with $\delta \ell=2$ requires a two-photon transition sufficiently detuned from the one intermediate state. We outline a scheme to generate these states in Appendix A. This scheme requires many pulses, but it is on par with previously proposed molecular dressing schemes [65-67] and may even be realized using recent experimental advancements [15].

We neglect rotational-state-dependent trapping effects, which are prominent in optical dipole traps [50,68-70]. These effects are negligible when considering a single molecule in the motional ground state of the trap, whose intensity can be robustly stabilized. In this case, the unique Stark shift for each rotational state due to the trap simply requires an updated microwave frequency catalog for all transitions. However, this spread in polarizability poses a practical problem when considering many molecules, since one must ensure that they all experience the same optical intensity. Accordingly, alternative trapping schemes may be more appropriate for the applications proposed in this work. Magnetic microtraps [46] are compatible with electronic spin doublet or triplet molecules such as $\mathrm{CaF}$, $\mathrm{SrF}, \mathrm{YbF}$, or YO. Radio-frequency electric traps are compatible with molecular ions [25,37]. Such trapping potentials are substantially less dependent on the rotational state of the molecule, since they couple to magnetic dipoles and electric monopoles, respectively.
More generally, one can consider engineering the desired pulses to generate states or correct errors via established optimal-control schemes [43,71]. It has been shown that one can control the planar [72,73], linear [7476], and even rigid [77] rotors, and it would be useful to extend these and other efforts $[78,79]$ to stabilizing the required code subspace.

\section{Crystal fields}

In a class of quantum error-correcting codes called stabilizer codes, the code space is the simultaneous eigenspace with eigenvalue 1 of a set of commuting Pauli operators, which are called check operators. A special subclass of stabilizer codes are the CSS codes, for which each check operator can be chosen to be either $Z$ type or $X$ type; the $Z$-type operators $\left\{\hat{S}_{Z}^{(i)}\right\}$ are diagonal in the computational basis, and the $X$-type operators $\left\{\hat{S}_{X}^{(j)}\right\}$ permute the computational basis states. The code subspace may be regarded as the degenerate ground space of the Hamiltonian (137)

$$
H_{\text {code }}=-\sum_{i} \hat{S}_{Z}^{(i)}-\sum_{j} \hat{S}_{X}^{(j)}
$$

Our molecular codes are not stabilizer codes, but, as we explain in Sec. V, the code space is the degenerate ground space of a Hamiltonian which is a sum of $Z$-type and $X$ type terms. Here, the $X$-type check operator rotates the molecule, while the $Z$-type check operator is diagonal in the position basis but alters the total angular momentum. Just like its oscillator counterpart (see Ref. [1], Sec. XIII), this molecular Hamiltonian is gapless, but ground states of an approximate gapped version would be close to the approximate code words we introduce in Sec. V C.

The $\hat{S}_{Z}$ check operators are momentum kicks which couple well-separated angular-momentum states $\left\{\left|{ }_{m n}^{\ell}\right\rangle\right\}$ for $\mathrm{SO}_{3}$ or $\left\{\left|m_{m}^{\ell}\right\rangle\right\}$ for $\mathrm{S}^{2}$. For example, $\hat{S}_{Z}$ for a linear-rotor code based on the octahedral group is a superposition of octopole $(\ell=4)$ spherical harmonics; see Eq. (116). Such harmonics are, in principle, present in a general interaction with a bath [80]. However, simple laser, dc, or microwave fields produce only $\ell \leq 2$ harmonics (see Ref. [61], Chaps. 4 and 7).

One way to generate the required higher value of $\ell$ is to put the molecule into a crystal lattice. For rotor codes based on a discrete subgroup $\mathrm{K} \subset \mathrm{SO}_{3}$, one such $\hat{S}_{Z}$ is the lowest$\ell$ function that is symmetric under $\mathrm{K}$. Thus, putting the rotor into a K-symmetric lattice yields a background field whose dominant term is exactly this $\hat{S}_{Z}$. For example, putting a linear rotor into an octahedrally symmetric lattice yields a background potential $[81,82]$ that is exactly the $\hat{S}_{Z}$ (116) required for the octahedral code. This potential is minimized at those orientations of the rotor that are superposed to construct the code words; in fact, these 
degenerate minima were noticed earlier in an experimental context [83]. Similarly, embedding into a two-dimensional square lattice yields the appropriate $\hat{S}_{Z}$ (114a) for a linearrotor version of the planar-rotor code introduced in Sec. IV. To access subgroups of $\mathrm{SO}_{3}$ forbidden in crystals, one could consider embedding a molecule in a quasicrystal.

Crystal symmetries can enforce only the $\hat{S}_{Z}$ check operator condition; the $\hat{S}_{X}$ check operator condition must be imposed by some other means. The $\hat{S}_{X}$ operators are trigonometric functions of the angular-momentum operators $\overleftarrow{\boldsymbol{L}}$ for $\mathrm{SO}_{3}$ or $\hat{\boldsymbol{L}}$ for $\mathrm{S}^{2}$. These are not naturally available, as the rigid-rotor Hamiltonian (75) and its generalizations [64] contain terms that are at most bilinear in the angularmomentum components. However, there are other terms in the full rotor-in-lattice Hamiltonian [see Ref. [84], Eq. (7.2)], and, akin to superconducting circuit schemes [85], one might engineer the molecule's environment (for example, by embedding the molecule in a liquid helium nanodroplet [86]) to provide the required $\hat{S}_{X}$ terms.

\section{Nuclear spin coupling}

If an error causes the molecule to rotate slightly, we recover from the error by applying a compensating small rotation. The desired rotation can be executed by turning on a Hamiltonian which is linear in the angular momentum. But, since the natural rigid-rotor Hamiltonian is quadratic, this linear term is not so easily realized in the laboratory.

One way to provide a Hamiltonian term which is linear in the molecule's angular momentum is to couple the rotational states of the molecule to nuclear spin states via nuclear spin-rotation interactions [see Ref. [60], Eq. (1.32)] $[48,51,87]$

$$
H_{\mathrm{nsr}}=\boldsymbol{I} \cdot \overleftarrow{\boldsymbol{L}}
$$

where $\boldsymbol{I}$ is the nuclear spin. The nuclear spin can serve as a convenient ancilla system, and the orientation of the molecule can be controlled by manipulating the nuclear spin. Similar approaches are applied to solid-state systems in which electronic spins are coupled to nuclear spins [88]. This approach is roughly analogous to using a superconducting Josephson-junction device coupled to a bosonic mode for manipulating the states of a bosonic errorcorrecting code.

We also need to correct momentum kicks by applying unitary operations that change the value of $\ell$. Operations which shift the occupation number of a cavity can be applied by coupling the cavity to a three-level atom [89] or by using linear optics [90]. Similar schemes could shift the value of $\ell$ for a $\mathrm{U}_{1}$ rotor. Extensions of such schemes may be helpful for controlling the rotational states of higherdimensional rotors.
TABLE I. Quotient spaces mentioned in this work [92,93] (see also Ref. [94], Sec. 3.8, and Refs. [95-97]). Spaces associated with $\mathrm{SO}_{3}$ characterize rotational states of various molecules (see Sec. III C). $\mathrm{Z}_{N}=\mathrm{C}_{N}$ is the order- $N$ cyclic group, $\mathrm{D}_{N}$ is the order$2 N$ dihedral group, $\mathrm{U}_{1}=\mathrm{SO}_{2}=\mathrm{C}_{\infty}$ is the circle group, and $\mathrm{O}_{2}=\mathrm{SO}_{2} \rtimes \mathrm{Z}_{2}=\mathrm{D}_{\infty}$ is the group of planar rotations and reflections. Some of these spaces are shown in Figs. 2 and 4.

\begin{tabular}{|c|c|c|}
\hline Space X & Group H & Quotient space $\mathrm{X} / \mathrm{H}$ \\
\hline $\mathrm{R}$ & $\mathrm{Z}$ & Wigner-Seitz unit cell $\mathrm{U}_{1}$ \\
\hline $\mathrm{SO}_{3}$ & $\begin{array}{c}\mathrm{Z}_{N} \\
\text { Dihedral } \mathrm{D}_{N} \\
\text { Tetrahedral T } \\
\text { Octahedral O } \\
\text { Icosahedral I } \\
\mathrm{U}_{1} \\
\mathrm{O}_{2}\end{array}$ & $\begin{array}{c}\text { Lens space } \mathrm{L}_{2 N, 1} \\
\text { Prism space } \\
\text { Octahedral space } \\
\text { Truncated cube space } \\
\text { Poincaré dodecahedral space } \\
\text { Two-sphere } \mathrm{S}^{2} \\
\text { Projective plane } \mathrm{RP}^{2}\end{array}$ \\
\hline$\overline{S^{2}}$ & $\mathrm{Z}_{N}, \mathrm{D}_{N}, \mathrm{~T}, \mathrm{O}, \mathrm{I}$ & Spherical two-orbifold \\
\hline
\end{tabular}

\section{B. Spin systems}

Certain combinations of spins offer another platform for simulating the linear-rotor space $S^{2}$ and quotient spaces $\mathrm{SO}_{3} / \mathrm{H}$ from Table I. We list three manifestations: $L$ spin$1 / 2$ systems in a totally symmetric spin state, $L$ spin- $N / 2$ systems in a totally symmetric state, and a pair of spin- $L / 2$ systems. In the limit of large $L$, each of these systems provides a useful approximation to one of the spaces of interest. While the first two cases are usually studied in the context of atomic ensembles, all three cases apply equally well to an atom or a molecule with a sufficiently large nuclear-spin manifold (cf. Ref. [91]).

\section{Many small spins}

$L$ spin- $1 / 2$ particles in a totally symmetric spin state have a total angular momentum of $L / 2$. The $L \rightarrow \infty$ limit of this large collective spin is sometimes said to be a semiclassical limit, meaning that the spin- $L / 2$ object behaves like a continuous classical spin when $L$ is large. An intuitive way to understand this limit is to consider the spin-coherent states

$$
\left.\left.|\mathbf{v}\rangle_{\mathrm{SC}}=\left(\left.e^{-i \varphi / 2} \cos \frac{\vartheta}{2}\right|_{1 / 2} ^{1 / 2}\right\rangle+\left.e^{i \varphi / 2} \sin \frac{\vartheta}{2}\right|_{-1 / 2} ^{1 / 2}\right\rangle\right)^{\otimes L}
$$

for $\mathbf{v}=(\vartheta, \varphi) \in \mathrm{S}^{2}[98,99]$. These states are not orthogonal; instead, they form an overcomplete frame for the collective spin's $(L+1)$-dimensional Hilbert space, with overlap $\left|\left\langle\mathbf{v} \mid \mathbf{v}^{\prime}\right\rangle_{\mathrm{SC}}\right|=\left(1+\mathbf{v} \cdot \mathbf{v}^{\prime} / 2\right)^{L}$. As $L \rightarrow \infty$, the states become orthogonal and correspond to the position states $|\mathbf{v}\rangle$ of $S^{2}$ (Table V B). For finite $L$, superpositions of these spincoherent states can be approximate code words for a linearrotor code.

Numerous manifestations of entangled ensembles of many spin- $1 / 2$ atoms have recently been demonstrated 
[100-103], and the current status of the field is summarized in Ref. [104].

\section{Many medium spins}

Any pure state of a spin- $1 / 2$ system is invariant under a continuous $\mathrm{U}_{1}$ subgroup of the rotation group $\mathrm{SO}_{3}$; each pure state corresponds to a point on the Bloch sphere, and a rotation about the axis aligned with that Bloch vector leaves the state invariant. In contrast, there are pure states in higher-spin representations for which the subgroup which preserves the state is a nontrivial discrete subgroup $\mathrm{H}$ of $\mathrm{SO}_{3}$. For example, the spin-2 state $\left.\left.\left.|\mathrm{T}\rangle \propto\right|_{-2} ^{2}\right\rangle+\left.\sqrt{2}\right|_{1} ^{2}\right\rangle$ is invariant under the tetrahedral subgroup $\mathrm{T}$. Therefore, applying $\mathrm{SO}_{3}$ rotations to $|\mathrm{T}\rangle$ generates a manifold of states $\left\{|a\rangle_{\mathrm{T}}\right\}$, where the label $a$ is a point in the coset space $a \in \mathrm{SO}_{3} / \mathrm{T}$. The spin-coherent states $\left\{|a\rangle_{\mathrm{T}}^{\otimes L}\right\}$, obtained by taking a tensor product of many identical elements of this manifold, approximate the position-basis states of $\mathrm{SO}_{3} / \mathrm{T}$ in the limit of large $L$. This idea can be generalized: Spincoherent states $\left\{|a\rangle_{\mathrm{H}}^{\otimes L}\right\}$ approximate the position-basis states for the coset space $\mathrm{SO}_{3} / \mathrm{H}$, if $|a\rangle_{\mathrm{H}}$ is a higher-spin state with invariance group $\mathrm{H}$.

The above T-symmetric and similar $\mathrm{H}$-symmetric states (see Ref. [105], Table 2)—examples of Perelomov coherent states [99] — are used as a mean-field ansatz for the ground space of spin- $N$ Bose-Einstein condensates [106,107]. We use such coherent states to extract error syndrome information for molecular codes (see Sec. V B), which requires projectively measuring in this basis [108].

\section{Two large spins}

Instead of using only the symmetric subspace, one can consider the entire space of a pair of spin- $L / 2$ systems. Per the addition rules (see Ref. [109], Chap. 8)

$$
L / 2 \otimes L / 2=0 \oplus 1 \oplus \cdots \oplus L,
$$

the $(L+1)^{2}$ orthonormal basis states for this system can be chosen to be the angular-momentum eigenstates $\left.\left\{||_{m}^{\ell}\right\rangle\right\}$, with $\ell \leq L$ and $|m| \leq \ell$. These are precisely the rotational states of a linear rotor, except for the truncation $\ell \leq L$. Formally, then, the state space of a pair of spin- $L / 2$ systems matches the state space on $S^{2}$ in the limit $L \rightarrow \infty$. Since the normalizable approximate code words of the linear-rotor code are necessarily truncated for large $L$ anyway, these approximate code words can be accurately realized using a pair of spin- $L / 2$ systems for sufficiently large $L$ (see Sec. V C).

If one instead considers two different spins $L / 2$ and $L^{\prime} / 2$, one obtains a different band of $S^{2}$ momentum states. While developing codes for such band-limited subspaces is outside the scope of this work, it is possible that our coding strategies may also be useful there.
As a concrete experimental platform for large-spin systems, we can consider nuclear spin spaces of molecules or single atoms. Diatomic molecules such as NaCs [24] offer exactly the band-limited subspaces mentioned above. Concerning single atoms, lanthanide species such as dysprosium (Dy), holmium (Ho), and erbium (Er) have large total spin manifolds in their ground states due to their large nuclear spins and many unpaired electrons in their $f$ shells. Accordingly, such atoms have already attracted attention for the possibility of scaling up quantum computing by collectively encoding in multilevel atoms [110112]. Ho, in particular, has the largest hyperfine ground space of any atom, with 128 ground states [112]. Laser cooling and trapping techniques are well established for Dy [113], Ho [114], and Er [115], as well as other lanthanides. Moreover, quantum degenerate gases of Dy $[116,117]$ and Er $[118,119]$ are widely used for novel quantum simulations based on their large magnetic dipole moments.

\section{Other systems \\ 1. Planar rotors}

Several systems have the configuration space of the planar rotor. The system depicted in Fig. 1(a) is a diatomic molecule confined to rotate in a two-dimensional plane, but one can also consider a two-ion crystal [120]. Other possibilities include the phase difference between two superconductors on either side of a Josephson junction [121], orbital angular momentum of light [122], or simulating rotor position states using phase states of an ordinary oscillator [123].

One can also embed the first few angular-momentum states of the planar rotor in the linear and rigid rotors. For fixed angular momentum $L$, the linear-rotor subspace $\left.\left\{||_{m}^{L}\right\rangle\right\}$ with $|m| \leq L$ is equivalent to the band-limited subspace $\{|\ell\rangle,|\ell| \leq L\}$ of the planar rotor.

\section{Symmetric molecules}

A molecule with symmetry group $\mathrm{H}$ has an orientation state space parameterized by $\mathrm{SO}_{3} / \mathrm{H}$ (see Table I). For example, the methane molecule $\mathrm{CH}_{3}$ has the tetrahedral symmetry group $\mathrm{T}$, and the alkaline earth monomethoxide $\left(\mathrm{MOCH}_{3}\right)$ family - potentially useful for quantum computing [26] - has symmetry group $Z_{3}$. This group is also the relevant symmetry group of Posner molecules, postulated to have potentially useful quantum effects $[124,125]$. The symmetry group of the fullerene molecule is the icosahedral group I, and the 3-manifold $\mathrm{SO}_{3} / \mathrm{I}$ has an exotic shape that was once proposed as a model for the geometry of the Universe $[97,126]$. It is interesting that such exotic spaces are readily accessible in relatively simple molecules. Completely asymmetric and $\mathrm{U}_{1}$-symmetric molecules correspond, respectively, to rigid and linear rotors from Sec. III A. 
More generally, if a group $\mathrm{G}$ acts transitively on the states of a quantum system, and the subgroup $\mathrm{H}$ of $\mathrm{G}$ leaves the states invariant, then the configuration space of the system is $\mathrm{G} / \mathrm{H}$. In Appendix D, we develop mathematical tools for parameterizing the position eigenstates and the dual momentum states of such a system, including orthogonality and completeness relations as well as a Poisson summation formula [127].

\section{Electronic states}

One can consider embedding certain spaces from Table I in the electronic eigenstates of single atoms. The eigenstates of hydrogen offer a platform for a band-limited subspace of the linear rotor $\mathrm{S}^{2}$ and even the space $\mathrm{SU}_{2}$ (closely related to the rigid rotor $\mathrm{SO}_{3}$; see Appendix B). Let us label the atom's eigenstates by $\left|\nu,{ }_{m}^{\ell}\right\rangle$, where $0 \leq|m| \leq$ $\ell<\nu$ and the energy $E_{\nu, \ell, m} \propto 1 / \nu^{2}$. For fixed energy $\nu=L$, the manifold of states is the same subspace of $\mathrm{S}^{2}$ as that obtained by combining two large spins in Eq. (6). If we instead consider all values of $\nu, \ell$, and $m$, we obtain $\mathrm{SU}_{2}$ by an appropriate unitary transformation, related to writing the hydrogen atom in parabolic coordinates [128].

\section{Vibrational states}

One can also consider using vibrational states of atoms or molecules to encode quantum information [34]. As control over vibrational states improves, it may be possible to implement bosonic error-correcting codes [3]. Position-state subspaces of harmonic oscillators also yield the two rotational spaces of interest. For example, considering position states $|x, y, z\rangle$ of three oscillators with $x^{2}+$ $y^{2}+z^{2}$ constant yields $\mathrm{S}^{2}$. With four oscillators, one obtains $\mathrm{SU}_{2}$.

To simulate $S^{2}$ using momentum states, one can take all Fock states of two oscillators with an even total occupation number. Such a simulation allows straightforward implementation of $\mathrm{SO}_{3}$ rotations via beam splitters.

\section{Levitated nanoparticles}

The code words of our $\mathrm{SO}_{3}$ and $\mathrm{S}^{2}$ codes are coherent superpositions of different possible orientations for a rigid body. Though we emphasize the potential applications to atoms and molecules, the same ideas can be applied to any quantized three-dimensional rigid body that can be coherently manipulated. While there is a size limitation due to decoherence, we are on the cusp of entering the quantum regime for levitated nanoscale particles of helium [129], vaterite [130], diamond (alone [131] or doped [132]), and silicon [133-135], to name a few. Nanoparticles may seem to be unlikely candidates for quantum computing, but it would be interesting nonetheless to try to stabilize quantum superpositions of their orientational states (cf. [136,137]).

\section{ERROR-CORRECTION BASICS FOR THE PLANAR ROTOR}

The goal of error correction is to encode quantum information into a cleverly chosen subspace (the code) such that it is possible to recover said information from errors caused by physical noise. Before proceeding to discuss codes which protect against noise acting on a three-dimensional rigid body, we review a simpler case which was previously considered in Ref. [1]: encoding a finite-dimensional system in the infinite-dimensional Hilbert space of a planar rotor. By discussing this case, we can introduce the key concepts underlying our code constructions in a familiar mathematical setting. The interested reader can consult Ref. [138] and Chap. 7 in Ref. [139] for other introductory material on quantum error correction, as well as related work on encodings associated with $\mathrm{U}_{1}[123,140]$.

The position-basis eigenstates for a planar rotor are in one-to-one correspondence with the elements of the twodimensional rotation group $\mathrm{U}_{1}=\mathrm{SO}_{2}=\mathrm{C}_{\infty}$. Equivalently, these are the position eigenstates for a particle moving on a circle; the basis elements may be denoted $\{|\phi\rangle, \phi \in$ $\left.[0,2 \pi)=\mathrm{U}_{1}\right\}$, with continuum normalization $\left\langle\phi \mid \phi^{\prime}\right\rangle=$ $\delta\left(\phi-\phi^{\prime}\right)$. A dual basis is provided by the angularmomentum eigenstates (also known as "rotational states") $\{|\ell\rangle, \ell \in \mathrm{Z}\}$, where $\langle\phi \mid \ell\rangle=(1 / \sqrt{2 \pi}) e^{i \ell \phi}$ and, hence, $\left\langle\ell \mid \ell^{\prime}\right\rangle=\delta_{\ell \ell^{\prime}}$.

Noise might rotate the system, applying an operator

$$
\hat{X}_{\phi^{\prime}}=e^{-i \phi^{\prime} \hat{L}}=\int_{\mathrm{U}_{1}} d \phi\left|\phi+\phi^{\prime}\right\rangle\langle\phi| ;
$$

alternatively, noise might kick the angular momentum, applying some power of the kick operator

$$
\hat{Z}=e^{i \hat{\phi}}=\sum_{\ell \in Z}|\ell+1\rangle\langle\ell| .
$$

In fact, we can expand an arbitrary noise channel $\mathcal{E}$ acting on the density operator $\rho$ of the planar rotor in terms of a complete basis of operators, where each element of the basis is a product of an $\hat{X}_{\phi}$ operator and an $\ell$ th power of the $\hat{Z}$ operator:

$$
\mathcal{E}(\rho)=\int_{\mathrm{U}_{1}^{\times 2}} d \phi d \phi^{\prime} \sum_{\ell, \ell^{\prime} \in \mathrm{Z}} \mathcal{E}_{\phi \phi^{\prime}}^{\ell \ell^{\prime}} \hat{X}_{\phi} \hat{Z}^{\ell} \rho \hat{Z}^{\ell^{\prime \dagger} \dagger} \hat{X}_{\phi^{\prime}}^{\dagger}
$$

Above, the expansion coefficients $\mathcal{E}_{\phi \phi^{\prime}}^{\ell \phi^{\prime}}$ are such that $\mathcal{E}$ is a quantum channel. Our goal is to encode a finite-dimensional logical system in the infinite-dimensional Hilbert space of the rotor, where this logical system is protected against any error $\hat{X}_{\phi} \hat{Z}^{\ell}$ where both $\phi$ and $\ell$ are sufficiently small. In other words, if $\rho$ consists of states in the logical (also known as code) subspace, and if $\mathcal{E}$ is expanded using 
only such correctable $\hat{X}_{\phi} \hat{Z}^{\ell}$, then we are able recover the original $\rho$ from $\mathcal{E}(\rho)$. Otherwise, recovery may not be possible, and logical information stored in $\rho$ may become corrupted.

\section{A. A protected qubit}

For example, the two orthonormal basis states of a protected qubit can be chosen to be [see Fig. 2(a)]

$$
\begin{aligned}
& |\overline{0}\rangle=\frac{1}{\sqrt{3}}\left(|\phi=0\rangle+\left|\phi=\frac{2 \pi}{3}\right\rangle+\left|\phi=\frac{4 \pi}{3}\right\rangle\right), \\
& |\overline{1}\rangle=\frac{1}{\sqrt{3}}\left(\left|\phi=\frac{\pi}{3}\right\rangle+|\phi=\pi\rangle+\left|\phi=\frac{5 \pi}{3}\right\rangle\right) .
\end{aligned}
$$

Both basis states are eigenstates with eigenvalue 0 of $\hat{\phi}$ modulo $\pi / 3$. Suppose that $|\bar{\psi}\rangle$ is an arbitrary state in the code space spanned by $|\overline{0}\rangle$ and $|\overline{1}\rangle$. If an error occurs which causes $\phi$ to shift by $\delta \phi \in[-\pi / 6, \pi / 6]$, we can unambiguously diagnose the error by measuring $\hat{\phi}$ modulo $\pi / 3$. Once $\delta \phi$ is known, we can correct the error by applying a unitary transformation that shifts $\phi$ by $-\delta \phi$, restoring the state of the rotor to the initial undamaged state $|\bar{\psi}\rangle$.

Alternatively, we may expand the basis states of the code in the angular-momentum eigenstate basis, finding

$$
\begin{aligned}
& \frac{1}{\sqrt{2}}(|\overline{0}\rangle+|\overline{1}\rangle)=\sqrt{\frac{3}{\pi}} \sum_{s \in \mathrm{Z}}|\ell=6 s\rangle, \\
& \frac{1}{\sqrt{2}}(|\overline{0}\rangle-|\overline{1}\rangle)=\sqrt{\frac{3}{\pi}} \sum_{s \in \mathrm{Z}}|\ell=6 s+3\rangle .
\end{aligned}
$$

Both basis states are eigenstates with eigenvalue 0 of $\hat{L}$ modulo 3. Suppose an error occurs which causes the angular momentum to shift by $\delta \ell \in\{-1,0,1\}$. We can unambiguously diagnose the error by measuring $\hat{L}$ modulo 3 . Once $\delta \ell$ is known, we can correct the error by applying a unitary transformation that shifts $\ell$ by $-\delta \ell$. Furthermore (see below), $\hat{\phi}$ modulo $\pi / 3$ and $\hat{L}$ modulo 3 are compatible observables that can be measured simultaneously. Therefore, we can correct any combination of shifts in $\phi$ and $\ell$, as long as the shift in $\phi$ is no larger than $\pi / 6$ and the shift in $\ell$ is no larger than 1 .

The code basis states in Eqs. (10) and (11) are not normalizable and, therefore, unphysical. However, we may replace the position eigenstates in Eq. (10) by narrow wave packets; then, the sum over $s$ in Eq. (11) is modulated by a broad envelope function. In that case, the code states are physical, and the nice error-correction properties we note still hold, up to negligibly small corrections.

Our main task in this paper is to generalize this code construction, in various directions. For that purpose, it is convenient to have other ways to describe the code.
Our first alternative description uses the stabilizer language [138,141].

\section{Stabilizer formalism}

A stabilizer code may be characterized as the simultaneous eigenspace with eigenvalue 1 of a set of commuting unitary operators, called the stabilizer generators. For the code specified by Eqs. (10) and (11), we may choose these operators to be

$$
\hat{S}_{Z} \equiv \hat{Z}^{6}=e^{i 6 \hat{\phi}}, \quad \hat{S}_{X} \equiv \hat{X}_{2 \pi / 3}=e^{-i(2 \pi / 3) \hat{L}} .
$$

To check that these operators commute, recall the relation $e^{i \hat{\phi}} \hat{L} e^{-i \hat{\phi}}=\hat{L}-1$ and the identity $\hat{X} e^{\alpha \hat{L}} \hat{X}^{\dagger}=e^{\alpha \hat{X} \hat{L} \hat{X}^{\dagger}}$ for any unitary $\hat{X}$ and scalar $\alpha . \hat{S}_{Z}$ and $\hat{S}_{X}$ are the code's check operators, which we can measure to diagnose errors. Note that measuring $\hat{S}_{Z}$ is equivalent to measuring $\hat{\phi}$ modulo $\pi / 3$ and that measuring $\hat{S}_{X}$ is equivalent to measuring $\hat{L}$ modulo 3, as we assert earlier, and that we can perform these measurements simultaneously because $\hat{S}_{Z}$ and $\hat{S}_{X}$ commute.

Furthermore, we note that the operators

$$
\bar{Z} \equiv \hat{Z}^{3}=e^{i 3 \hat{\phi}}, \quad \bar{X}=\hat{X}_{\pi / 3}=e^{-i(\pi / 3) \hat{L}}
$$

also commute with the stabilizer generators, which means that these are logical operators which preserve the code space. We see also that $\bar{Z}$ and $\bar{X}$ anticommute and that they square to the identity on the code space, where $\hat{S}_{Z}=\hat{S}_{X}=1$. Thus, $\bar{Z}$ and $\bar{X}$ may be regarded as the logical Pauli operators acting on the encoded qubit, where $\bar{Z}$ is diagonal in the basis $\{|\overline{0}\rangle,|\overline{1}\rangle\}$ and $\bar{X}$ is diagonal in the conjugate basis $\{(1 / \sqrt{2})(|\overline{0}\rangle \pm|\overline{1}\rangle)\}$.

\section{CSS construction}

We may also describe our protected qubit using the language of CSS codes $[138,141]$. In the CSS construction, a quantum error-correcting code is built from a classical error-correcting code $\mathrm{K}$ and a subcode $\mathrm{H} \subset \mathrm{K}$.

In the case of the protected qubit with basis states (10), the code $\mathrm{K}$ is a six-state system embedded in the infinitedimensional Hilbert space of the rotor, with the six states corresponding to six equally spaced angular positions of the rotor, rotated by $\phi=(2 \pi / 6) k, k \in\{0,1, \ldots, 5\}$, relative to a standard reference orientation. This classical system is protected against errors that shift the rotor slightly, rotating it through an angle $\delta \phi \in[-\pi / 6, \pi / 6]$. The subcode $\mathrm{H}$ has three states, with orientations $\phi=(2 \pi / 3) k, k \in\{0,1,2\}$, and protects against rotations which are twice as large: $\delta \phi \in[-\pi / 3, \pi / 3]$. In the associated quantum code, each of the basis states (10) is a uniform superposition of all the elements of a coset of $\mathrm{H}$ in $\mathrm{K}$, the trivial coset (the elements of $\mathrm{H}$ ) for the basis state $|\overline{0}\rangle$, and the nontrivial coset for the 
basis state $|\overline{1}\rangle$. The protection of this qubit against shifts of the rotor is inherited from the corresponding property of the classical code $\mathrm{K}$.

There is a dual description of this quantum code, making use of the angular-momentum basis of the rotor rather than its position basis. The classical code $\mathrm{H}^{\perp}$, dual to $\mathrm{H}$, contains all angular-momentum eigenstates where $\ell$ is an integer multiple of 3 . These two classical codes are dual in the sense that the representations of the group $\mathrm{U}_{1}$ contained in $\mathrm{H}^{\perp}$ represent the elements of $\mathrm{H}$ trivially. Similarly, the classical code $\mathrm{K}^{\perp}$ dual to $\mathrm{K}$ contains all angular-momentum eigenstates where $\ell$ is an integer multiple of 6 , those representations which represent $\mathrm{K}$ trivially. Evidently, $\mathrm{K}^{\perp}$ is a subcode of $\mathrm{H}^{\perp}$. For the quantum code, each basis state in Eq. (11) is a uniform superposition of all the elements of a coset of $\mathrm{K}^{\perp}$ in $\mathrm{H}^{\perp}$, the trivial coset for the basis state $1 / \sqrt{2}(|\overline{0}\rangle+|\overline{1}\rangle)$, and the nontrivial coset for the basis state $1 / \sqrt{2}(|\overline{0}\rangle-|\overline{1}\rangle)$. The classical code $\mathrm{H}^{\perp}$ protects against shifts of the angular momentum by $\delta \ell \in\{-1,0,1\}$, and the quantum code inherits this property.

Viewed as an abstract group, the code $\mathrm{K}$ is the subgroup $\mathrm{Z}_{6}$ of $\mathrm{U}_{1}$, and $\mathrm{H}$ is the subgroup $\mathrm{Z}_{3} \subset \mathrm{Z}_{6}$. The construction can be easily generalized to $\mathrm{K}=\mathrm{Z}_{d N}$ and $\mathrm{H}=\mathrm{Z}_{N}$, where $d$ and $N$ are positive integers, in which case the quantum code is $d$-dimensional. In the stabilizer language, this more general code has stabilizer generators

$$
\hat{S}_{Z}=\hat{Z}^{d N}, \quad \hat{S}_{X}=\hat{X}_{2 \pi / N} .
$$

Its logical operators

$$
\bar{Z}=\hat{Z}^{N}, \quad \bar{X}=\hat{X}_{2 \pi / d N}
$$

are generalized Pauli operators, obeying the HeisenbergWeyl commutation relation $\bar{Z} \bar{X}=e^{i(2 \pi / d)} \bar{X} \bar{Z}$. This quantum code protects against position shifts by $\delta \phi$ with $|\delta \phi|<$ $(\pi / d N)$ and momentum kicks by $\delta \ell$ with $|\delta \ell| \leq(N-1) / 2$ (for odd $N$ ). Note the trade-off: Increasing $N$ improves the protection against angular-momentum kicks but weakens the protection against rotations.

\section{Partial Fourier transform}

There is yet another way to describe the code construction, using the notion of a partial Fourier transform, which is helpful as we seek further generalizations. Recall that the position and angular-momentum bases for the planar rotor are related by Fourier transforming:

$$
\begin{aligned}
& |\ell\rangle=\int_{-\pi}^{\pi} d \phi|\phi\rangle\langle\phi \mid \ell\rangle=\frac{1}{\sqrt{2 \pi}} \int_{-\pi}^{\pi} d \phi|\phi\rangle e^{i \ell \phi}, \\
& |\phi\rangle=\sum_{\ell \in \mathrm{Z}}|\ell\rangle\langle\ell \mid \phi\rangle=\frac{1}{\sqrt{2 \pi}} \sum_{\ell \in \mathrm{Z}}|\ell\rangle e^{-i \ell \phi} .
\end{aligned}
$$

It is useful to imagine that the above integral over $\phi$ is carried out in two steps. We write $\phi=a+(2 \pi / N) h$, where $a \in(-\pi / N, \pi / N]$ and $h \in\{0,1, \ldots, N-1\}$; then, integrating $\phi$ from $-\pi$ to $\pi$ is equivalent to integrating $a$ from $-\pi / N$ to $\pi / N$ and summing $\mathrm{H}$ from 0 to $N-1$. Likewise, we can do the sum over $\ell$ in two steps; we write $\ell=N s+\lambda$, where $s \in \mathrm{Z}$ and $\lambda \in\{0,1, \ldots, N-1\}$, and we separate the infinite sum over $s$ from the finite sum over $\lambda$. When we speak of a "partial Fourier transform," we mean performing one of these two steps without the other.

By performing the sum over $\mathrm{H}$ but not the integral over $a$, we obtain a new orthonormal basis

$$
\begin{aligned}
|a ; \lambda\rangle & \equiv \frac{1}{\sqrt{N}} \sum_{h \in Z_{N}} e^{i(2 \pi / N) \lambda h}\left|\phi=a+\frac{2 \pi}{N} h\right\rangle \\
& =e^{-i \lambda a} \sqrt{\frac{N}{2 \pi}} \sum_{s \in \mathrm{Z}} e^{-i N s a}|\ell=N s+\lambda\rangle,
\end{aligned}
$$

with normalization $\left\langle a ; \lambda \mid a^{\prime} ; \lambda^{\prime}\right\rangle=\delta\left(a-a^{\prime}\right) \delta_{\lambda \lambda^{\prime}}$. From now on, the presence of a semicolon inside a ket declares that ket to be an element of this basis.

This $\{|a ; \lambda\rangle\}$ basis is convenient for our purposes, because shifts in position or angular momentum affect only one of the two indices. A shift in angular momentum by $\delta \ell$ acts on the basis according to

$$
|a ; \lambda\rangle \rightarrow|a ;(\lambda+\delta \ell) \bmod N\rangle
$$

(up to a phase), shifting $\lambda \rightarrow \lambda+\delta \ell$ modulo $N$. A shift in position by $\delta \phi$ shifts $a \rightarrow a+\delta \phi$ modulo $2 \pi / N$ :

$$
|a ; \lambda\rangle \rightarrow\left|(a+\delta \phi) \bmod \frac{2 \pi}{N} ; \lambda\right\rangle .
$$

To recover our previous code construction, we choose $d$ basis states $\{|\bar{k}\rangle\}$ with $\lambda=0$ and $a=(2 \pi / d N) k$, finding

$$
\begin{aligned}
|\bar{k}\rangle=\left|\frac{2 \pi}{d N} k ; 0\right\rangle & =\frac{1}{\sqrt{N}} \sum_{h \in Z_{N}}\left|\phi=\frac{2 \pi}{d N} k+\frac{2 \pi}{N} h\right\rangle \\
& =\sqrt{\frac{N}{2 \pi}} \sum_{s \in \mathrm{Z}} e^{-i(2 \pi / d) s k}|\ell=N s\rangle .
\end{aligned}
$$

If an error occurs in which $|\delta \ell| \leq(N-1) / 2$ (for odd $N$ ) and $|\delta \phi|<(\pi / d N)$, we diagnose the error by performing a measurement which determines the value of $\lambda$ and also the value of $a(\bmod 2 \pi / d N)$. Then, the value of $a$ unambiguously identifies the shift in $\phi$, and the value of $\lambda$ unambiguously identifies the shift in $\ell$. Once known, these shifts can be corrected to recover the initial undamaged code states.

The orientation label $\phi$ of the planar rotor can be viewed as the element of the group $\mathrm{U}_{1}$ describing the rotation which reaches $\phi$ starting from a standard initial orientation. 
The basis $\{|a ; \lambda\rangle\}$ for the rotor's Hilbert space reflects a decomposition of $U_{1}$ which may be written symbolically as

$$
\mathrm{U}_{1} \cong \mathrm{U}_{1} / \mathrm{Z}_{N} \times \widehat{\mathrm{Z}_{N}}
$$

That is, $a$ labels an element of $\mathrm{U}_{1} / \mathrm{Z}_{N}$ (a coset of $\mathrm{Z}_{N}$ in $\mathrm{U}_{1}$ ), and $\lambda$ labels an element of $\widehat{Z_{N}}$ (an irreducible representation of $Z_{N}$ ). Our error-correction procedure makes use of a finer decomposition:

$$
\mathrm{U}_{1} \cong \mathrm{U}_{1} / \mathrm{Z}_{d N} \times \mathrm{Z}_{d N} / \mathrm{Z}_{N} \times \widehat{\mathrm{Z}_{N}} .
$$

The correctable rotation error is an element of $\mathrm{U}_{1} / \mathrm{Z}_{d N}$, the correctable angular-momentum kick is an element of $\widehat{Z_{N}}$, and code basis states correspond to elements of $\mathrm{Z}_{d N} / \mathrm{Z}_{N}$. We use similar decompositions in our constructions of quantum codes for more general groups.

\section{B. Gates, recovery, and initialization}

To use the above codes for quantum computation on multiple encoded rotors, we need to initialize in the code subspace, execute quantum gates, and perform the measurement-based error correction described above. For these tasks, we need operators other than the Pauli-type operators $\hat{X}_{\phi}(7)$ and $\hat{Z}^{\ell}(8)$. As is typical of quantum codes, there is an "easy" subset of all possible operators that aid us in the above tasks in a reasonably fault-tolerant manner. For $\mathrm{U}_{1}$ rotors, such normalizer or symplectic operations are generated by certain quadratic functions of the rotors' positions and momenta $[142,143]$.

\section{Symplectic operations}

Single-rotor symplectic operations include unitary operators generated by Hamiltonians that are polynomials in angular momentum of at most degree 2 . The quadraticphase operator $\operatorname{QUAD}_{\varphi}=e^{-i \varphi \hat{L}(\hat{L}+1) / 2}$ (with angle $\varphi \in \mathrm{U}_{1}$ ) maps

$$
\hat{Z} \rightarrow \hat{X}_{\varphi} \hat{Z}
$$

while commuting with position shifts $\hat{X}_{\phi}$ (also generated by $\hat{L})$. The analogous two-rotor "conditional-phase" operator $\mathrm{CPHS}_{\varphi}=e^{-i \varphi \hat{L} \otimes \hat{L}}$ [cf. [123], Eq. (23)] commutes with $\hat{X}_{\phi} \otimes 1$ and $1 \otimes \hat{X}_{\phi}$ but maps

$$
\hat{Z} \otimes 1 \rightarrow \hat{Z} \otimes \hat{X}_{\varphi} \quad \text { and } \quad 1 \otimes \hat{Z} \rightarrow \hat{X}_{\varphi} \otimes \hat{Z} .
$$

Another operation is the conditional rotation

$$
\mathrm{CROT} \equiv e^{-i \hat{\phi} \otimes \hat{L}}=\int_{\mathrm{U}_{1}} d \phi|\phi\rangle\langle\phi| \otimes \hat{X}_{\phi},
$$

shifting the position of the second rotor by $\phi$, conditioned on the first rotor being at position $\phi$. This maps

$$
\hat{X}_{\phi} \otimes 1 \rightarrow \hat{X}_{\phi} \otimes \hat{X}_{\phi} \quad \text { and } \quad 1 \otimes \hat{Z} \rightarrow \hat{Z}^{\dagger} \otimes \hat{Z}
$$

while acting trivially on $\hat{Z} \otimes 1$ and $1 \otimes \hat{X}_{\phi}$.

The QUAD and CPHS operations can be realized by turning on Hamiltonians quadratic in angular momenta for a specified amount of time [cf. Eq. (4)]. The CROT operation, however, cannot be obtained from the "Hamiltonian" $H=\hat{\phi} \otimes \hat{L}$, because such an $\mathrm{H}$ would not be invariant under $2 \pi$ rotations of the first rotor and, therefore, would not be single valued. (A similar problem plagues the Hamiltonian $\hat{\phi}$, present in the exponent of $\hat{Z}$, while $\hat{\phi}^{2}$ is not single valued even when exponentiated.) To produce such an operator in the lab, one can consider adapting implementations of the related oscillator phase operator to rotors $[89,90]$ (see Sec. III A).

\section{Logical gates}

The above symplectic operations, for certain $\varphi$, perform logical Clifford operations on the encoded qudits. The gate $\mathrm{QUAD}_{2 \pi / d N^{2}}$ performs a logical qudit rotation mapping $\bar{Z} \rightarrow$ $\overline{X Z}$ (up to a phase), while $\mathrm{CPHS}_{2 \pi / d N^{2}}$ and CROT act as entangling gates.

In the case of a logical qubit $(d=2)$ with logical operators $\bar{Z}=\hat{Z}^{N}$ and $\bar{X}=\hat{X}_{\pi / N}$ (15), the symplectic operations producing the above logical transformations act on the rotor positions $\phi_{1,2}$ and momenta $\ell_{1,2}$ as follows:

$$
\begin{aligned}
\operatorname{QUAD}_{\pi / N^{2}}: \phi & \rightarrow \phi-\frac{\pi}{N^{2}} \ell+c, & \ell & \rightarrow \ell, \\
\mathrm{CPHS}_{\pi / N^{2}}: \phi_{1} & \rightarrow \phi_{1}-\frac{\pi}{N^{2}} \ell_{2}, & \ell_{1} & \rightarrow \ell_{1}, \\
\phi_{2} & \rightarrow \phi_{2}-\frac{\pi}{N^{2}} \ell_{1}, & \ell_{2} & \rightarrow \ell_{2}, \\
\text { CROT }: \phi_{1} & \rightarrow \phi_{1} & \ell_{1} & \rightarrow \ell_{1}-\ell_{2}, \\
\phi_{2} & \rightarrow \phi_{2}+\phi_{1}, & \ell_{2} & \rightarrow \ell_{2},
\end{aligned}
$$

with constant $c=(\pi / 2)\left(N-1 / N^{2}\right)$ (cf. Sec. IX in Ref. [1]). We assume in Eq. (27) that $\phi$ and $\ell$ simultaneously have definite values, which makes sense for an encoded state assuming that $\phi$ and $\ell$ are sufficiently small. These transformations do not amplify correctable position and momentum shifts into uncorrectable ones, and a small overrotation or underrotation in the implementation of one of the logical gates introduces only correctable errors, not logical errors. In this sense, the logical gates are fault tolerant.

The above symplectic operations do not provide a universal set of logical operations. One way to upgrade to such a set is to include unitaries generated by the logical operators $\{\bar{X}, \bar{Z}\}$ themselves. The gates $e^{i \varphi(\bar{X}+\text { H.c. })}$ and $e^{i \varphi^{\prime}(\bar{Z}+\text { H.c. })}$ allow for arbitrary single-qudit rotations, while $e^{i \varphi^{\prime \prime}(\bar{X} \otimes \bar{X}+\text { H.c. })}$ allows for arbitrary logical $X X$ rotations. Such gates are, however, not fault tolerant, as fluctuations 
in the $\varphi$ 's produce undetectable errors. One can also consider using Hamiltonians that are cubic (or higher) in angular momenta.

\section{Diagnosis and recovery}

A shift in the position by $\delta \phi$ and momentum by $\delta \ell$ maps logical states $|\bar{k}\rangle \rightarrow|(2 \pi / d N) k+\delta \phi ; \delta \ell\rangle$ (up to a phase). To diagnose the errors, we need to measure $\hat{\phi} \bmod 2 \pi / d N$ and $\hat{L} \bmod N$. Once this error syndrome is known, we can undo the damage by applying $\hat{X}_{\delta \phi}^{\dagger}$ (7) and $\hat{Z}^{\delta \ell \dagger}$ (8) to the corrupted logical states.

To measure $\hat{\phi} \bmod 2 \pi / d N$, we need an ancilla that can resolve all possible values of this syndrome while revealing no information about the protected encoded state. One way to extract the syndrome is to encode the ancilla using the same code that protects the data [144]. Specifically, we may prepare an ancillary rotor in the logical- $X$ eigenstate $\left|\overline{0}_{X}\right\rangle$, a uniform superposition of the position eigenstates $\left\{\left|\phi=(2 \pi / d N) k^{\prime}\right\rangle, k^{\prime}=0,1, \ldots, d N-1\right\}$, which is, therefore, invariant under the rotation $\phi \rightarrow \phi+(2 \pi / d N)$. Applying the CROT gate (25) to a noisy logical state and a noiseless ancilla yields

$$
\begin{aligned}
& \text { CROT }\left|\frac{2 \pi}{d N} k+\delta \phi ; \delta \ell\right\rangle \otimes\left|\overline{0}_{X}\right\rangle \\
& =\left|\frac{2 \pi}{d N} k+\delta \phi ; \delta \ell\right\rangle \otimes \hat{X}_{\delta \phi}\left|\overline{0}_{X}\right\rangle .
\end{aligned}
$$

The ancilla can then be measured in the $\{|\phi\rangle\}$ basis, and the measured value modulo $2 \pi / d N$ determines the shift $\delta \phi$. If the ancilla is noisy or the measurement is imperfect, then the extracted value of $\delta \phi$ is likewise noisy; nevertheless, if a fresh supply of ancilla rotors is continuously available, this recovery procedure with high likelihood prevents small displacements of the data rotor from accumulating to produce an uncorrectable logical error.

To measure $\hat{L} \bmod N$, we need an ancilla that can resolve the $N$ values of the syndrome. In this case, we could initialize an ancilla rotor in the state $|\phi=0\rangle$ and apply $\mathrm{CPHS}_{2 \pi / N}$ to the data and ancilla rotors. This gate rotates the ancilla by $(2 \pi / N) \delta \ell$, and the value of $\delta \ell$ can, therefore, be extracted by measuring the ancilla in the position basis. Since the syndrome takes discrete values, some noise resilience is built into the procedure $-\delta \ell$ is determined by rounding off the measured value of $\phi$ to the nearest multiple of $2 \pi / N$.

Since we need only to resolve a discrete number of momentum syndrome values, a discretized version of the above scheme using a qunit ancilla works just as well. Let $\left\{\left|h_{z}\right\rangle, h \in \mathrm{Z}_{N}\right\}$ be the position states of the qunit, and initialize the qunit in the state $\left|0_{z}\right\rangle$. Then apply the entangling gate

$$
\mathrm{CPHS}^{\prime} \equiv \sum_{\ell \in \mathrm{Z}}|\ell\rangle\langle\ell| \otimes \mathcal{X}^{\ell}
$$

where $\mathcal{X}$ satisfies $\mathcal{X}\left|h_{z}\right\rangle=\left|h+1_{z}\right\rangle$ (modulo $N$ ) and $\mathcal{X}^{N}$ is the identity. This process yields

$\mathrm{CPHS}^{\prime}\left|\frac{2 \pi}{d N} k+\delta \phi ; \delta \ell\right\rangle \otimes\left|0_{z}\right\rangle=\left|\frac{2 \pi}{d N} k+\delta \phi ; \delta \ell\right\rangle \otimes\left|\delta \ell_{z}\right\rangle$,

and measuring the qunit in the position basis then reveals the syndrome.

\section{Initialization}

The above error-correction procedures can equivalently be used to initialize in certain logical states. For example, consider one rotor initialized in $|\phi=0\rangle$, coupled to an ancillary qunit initially in $\left|0_{z}\right\rangle$. Applying CPHS $^{\prime}$ yields

$$
\mathrm{CPHS}^{\prime}|\phi=0\rangle \otimes\left|0_{z}\right\rangle \propto \sum_{\lambda \in Z_{N}}|0 ; \lambda\rangle \otimes\left|\lambda_{z}\right\rangle .
$$

Measuring the ancilla in the $\left|h_{z}\right\rangle$ basis to obtain a particular $\lambda=\lambda_{\star}$ collapses the rotor state to $\left|0 ; \lambda_{\star}\right\rangle$. Applying a momentum kick $\hat{Z}^{\lambda_{\star} \dagger}$ then produces the logical state $|\overline{0}\rangle=|0 ; 0\rangle$ (20), thereby completing the initialization. Analogous initialization schemes use the position syndrome measurement.

\section{MOLECULAR CODES}

By a "molecular code," we mean a finite-dimensional subspace of the infinite-dimensional Hilbert space of a rigid body in three dimensions (also known as a "rigid rotor"). To define a basis for this infinite-dimensional Hilbert space, we imagine fixing a coordinate system in the laboratory, pinning the body's center of mass, and specifying the orientation of the body relative to a standard initial configuration in this fixed coordinate system. For a molecule with no symmetries, the possible orientations are in one-to-one correspondence with the elements of the $3 \mathrm{D}$ special orthogonal group $\mathrm{SO}_{3}$; thus, we may choose the "position" basis $\left\{|R\rangle, R \in \mathrm{SO}_{3}\right\}$. This correspondence between group elements and orientations of the body follows the same logic as in our discussion of the planar rotor in Sec. IV, where we identify position-basis eigenstates with elements of $\mathrm{U}_{1}$.

If the body has symmetries, using a group element to specify the orientation becomes redundant, and the position basis should be refined accordingly. For example, if there is an axis of symmetry (as for a diatomic molecule composed of two distinct nuclei), the body is invariant under the $\mathrm{U}_{1}$ subgroup of rotations about the symmetry axis, and the possible orientations are in one-to-one correspondence with the coset space $\mathrm{SO}_{3} / \mathrm{U}_{1}$, which is equivalent to the 
two-sphere $\mathrm{S}^{2}$. If, in addition, the axis of symmetry has no preferred direction, so the body is invariant under reflections that invert the axis (as for a diatomic molecule composed of two identical nuclei), then the position eigenstates are labeled by the elements of the real projective space $\mathrm{SO}_{3} / \mathrm{O}_{2}=\mathrm{RP}^{2}$. In this section, we assume the body has no symmetries; the case of a body with a symmetry axis (also known as a "linear rotor") is discussed in Sec. VI.

An active rotation of the body described by $\mathrm{SO}_{3}$ element $S$ alters the orientation of the body according to $R \rightarrow S R$; that is, the group element describing the orientation of the body is left multiplied by $S$. This rotation is represented by the unitary operator $\vec{X}_{S}$, which acts on the Hilbert space according to

$$
\vec{X}_{S}:|R\rangle \rightarrow|S R\rangle .
$$

We also consider passive rotations, rotations of the coordinate system in the laboratory, which act on the position basis according to

$$
\overleftarrow{X}_{S}:|R\rangle \rightarrow\left|R S^{-1}\right\rangle
$$

As in our discussion of the $\mathrm{U}_{1}$ case, there is a Fourierconjugate basis of angular-momentum states (also known as "rotational states"), defined for $\mathrm{SO}_{3}$ by

$$
\begin{aligned}
& \left|{ }_{m n}^{\ell}\right\rangle=\int_{\mathrm{SO}_{3}} d R \sqrt{\frac{2 \ell+1}{8 \pi^{2}}} D_{m n}^{\ell}(R)|R\rangle, \\
& \left.|R\rangle=\left.\sum_{\ell \geq 0} \sum_{|m|,|n| \leq \ell} \sqrt{\frac{2 \ell+1}{8 \pi^{2}}} D_{m n}^{\ell \star}(R)\right|_{m n} ^{\ell}\right\rangle .
\end{aligned}
$$

The $\left\{D_{m n}^{\ell}\right\}$ denote matrix elements of the angular momentum $\ell$ irreducible representation of $\mathrm{SO}_{3}$, obeying

$$
D_{m n}^{\ell}(S R)=\sum_{p} D_{m p}^{\ell}(S) D_{p n}^{\ell}(R)
$$

and $D_{m n}^{\ell}\left(R^{-1}\right)=D_{n m}^{\ell \star}(R)$, with normalization [145]

$$
\int_{\mathrm{SO}_{3}} d R D_{m n}^{\ell \star}(R) D_{m^{\prime} n^{\prime}}^{\ell^{\prime}}(R)=\frac{8 \pi^{2}}{2 \ell+1} \delta_{\ell \ell^{\prime}} \delta_{m m^{\prime}} \delta_{n n^{\prime}}
$$

The integral is with respect to the invariant Haar measure $d R$ on $\mathrm{SO}_{3}$, here normalized so that the volume of the group $\int_{\mathrm{SO}_{3}} d R=8 \pi^{2}$.

The elements of the conjugate basis transform under active and passive rotations according to

$$
\begin{aligned}
& \left.\vec{X}_{R}\left|\begin{array}{l}
\ell \\
m n
\end{array}\right\rangle=\left.\sum_{p} D_{p m}^{\ell \star}(R)\right|_{p n} ^{\ell}\right\rangle, \\
& \left.\overleftarrow{X}_{R}\left|\begin{array}{l}
\ell \\
m n
\end{array}\right\rangle=\left.\sum_{p} D_{p n}^{\ell}(R)\right|_{m p} ^{\ell}\right\rangle .
\end{aligned}
$$

These and other useful properties of the $D_{m n}^{\ell}$ matrices are summarized in Table IV.

The functions $D_{m n}^{\ell}(R)$ are a complete basis for functions which map the group to complex numbers. Hence, an arbitrary function $f(R)$ on the group can be Fourier expanded in this basis:

$$
\begin{aligned}
f(R) & =\sum_{\ell \geq 0} \sum_{|m|,|n| \leq \ell} \sqrt{\frac{2 \ell+1}{8 \pi^{2}}} f_{m n}^{\ell} D_{m n}^{\ell}(R), \\
f_{m n}^{\ell} & =\int_{\mathrm{SO}_{3}} d R \sqrt{\frac{2 \ell+1}{8 \pi^{2}}} D_{m n}^{\ell \star}(R) f(R) .
\end{aligned}
$$

We use the notation $\hat{f}$ for the operator associated with the function $f(R)$ that is diagonal in the $\{|R\rangle\}$ basis:

$$
\hat{f}=\int_{\mathrm{SO}_{3}} d R|R\rangle f(R)\langle R| .
$$

Fourier expanding this operator, we obtain

$$
\hat{f}=\sum_{\ell \geq 0} \sum_{|m|,|n| \leq \ell} \sqrt{\frac{2 \ell+1}{8 \pi^{2}}} f_{m n}^{\ell} \hat{D}_{m n}^{\ell} .
$$

An arbitrary operator $E$ acting on the Hilbert space of the rotor can be expanded in terms of an operator basis, in which each element of the basis is a diagonal operator followed by an active rotation. After Fourier expanding the diagonal operator, $E$ has the expansion

$$
E=\int_{\mathrm{SO}_{3}} d S \sum_{\ell \geq 0} \sum_{|m|,|n| \leq \ell} E_{m n}^{\ell}(S) \vec{X}_{S} \hat{D}_{m n}^{\ell},
$$

where we absorb an $\ell$-dependent numerical factor into the coefficient $E_{m n}^{\ell}(S)$. This result is the analog of the expansion of an operator acting on a qudit in terms of the Pauli operator basis. Therefore, a completely positive noise channel $\mathcal{E}$ acting on a state $\rho$ of the rigid rotor has an expansion of the form

$$
\begin{aligned}
\mathcal{E}(\rho)= & \int_{\mathrm{SO}_{3}} d S \int_{\mathrm{SO}_{3}} d S^{\prime} \sum_{\ell, m, n} \sum_{\ell^{\prime}, m^{\prime}, n^{\prime}} \mathcal{E}_{m n m^{\prime} n^{\prime}}^{\ell} \underset{\ell^{\prime}}{\ell^{\prime}}\left(S, S^{\prime}\right) \\
& \times \vec{X}_{S} \hat{D}_{m n}^{\ell} \rho \hat{D}_{m^{\prime} n^{\prime}}^{\ell^{\prime} \dagger} \vec{X}_{S^{\prime}}^{\dagger} .
\end{aligned}
$$

Our goal is to construct a code that allows us to recover successfully from any error of the form

$$
\rho \rightarrow \vec{X}_{S} \hat{D}_{m n}^{\ell} \rho \hat{D}_{m^{\prime} n^{\prime}}^{\ell^{\prime \dagger}} \vec{X}_{S^{\prime}}^{\dagger}
$$

where the position shifts $S$ and $S^{\prime}$ and momentum kicks $\ell$ and $\ell^{\prime}$ are sufficiently small. Using this code, we can recover from the noise channel $\mathcal{E}$ with high fidelity if 
$\vec{X}_{S} \hat{D}_{m n}^{\ell} \rho \hat{D}_{m^{\prime} n^{\prime}}^{\ell^{\prime \dagger}} \vec{X}_{S^{\prime}}^{\dagger}$ has most of its support on small values of $S, S^{\prime}, \ell$, and $\ell^{\prime}$.

To determine how well these codes protect against physical rigid-rotor noise models [146-152], one expands the noise operators $E$ from Eq. (41) in terms of position and momentum shifts and estimates the probability of an uncorrectable error. A similar analysis for code states of harmonic oscillators rather than rotors (see Ref. [3], Sec. VII) shows that physically relevant noise is typically correctable with high probability. If the noise acts locally in phase space, our rotor codes should perform well.

\section{A. $\mathbf{Z}_{N} \subset \mathbf{Z}_{2 N}$ codes}

We want to construct a finite-dimensional code subspace for the rigid rotor which is protected against small shifts in position and in angular momentum. For this purpose, we specify a discrete subgroup $\mathrm{H} \subset \mathrm{G}=$ $\mathrm{SO}_{3}$ and consider the basis defined by the corresponding partial Fourier transform. In this section, we assume $\mathrm{H}$ is $\mathrm{Z}_{N}$, an Abelian group of rotations about the $\mathbf{z}$ axis; the case where $\mathrm{H}$ is non-Abelian is discussed in Secs. VD and VE.

As for the case of $G=U_{1}$ from Sec. IVA, our code construction makes use of a basis defined by a partial Fourier transform associated with the subgroup $Z_{N}$. The elements of this basis are

$$
\left|S \mathrm{Z}_{N} ; \lambda\right\rangle \equiv \frac{1}{\sqrt{N}} \sum_{h \in Z_{N}} e^{i(2 \pi / N) \lambda h} \vec{X}_{S}\left|\frac{2 \pi}{N} h, \mathbf{z}\right\rangle
$$

here, $|\omega, \mathbf{z}\rangle$ denotes the position eigenstate $|R\rangle$, where $R \equiv$ $R_{\omega, \mathbf{z}}$ is a rotation by angle $\omega \in[0,2 \pi)$ about the $\mathbf{z}$ axis. Note that the index $\lambda \in\{0,1, \ldots, N-1\}$ indicates the irreducible representation of $\mathrm{Z}_{N}$ according to which the state $\left|S Z_{N} ; \lambda\right\rangle$ transforms. From now on, the presence of a semicolon inside a ket declares that ket to be an element of this basis.

The rotation $S$ is a representative of a coset in the lens space $\mathrm{SO}_{3} / \mathrm{Z}_{N}$. Coset representatives are not unique because of the freedom to multiply $S$ on the right by an element of $\mathrm{Z}_{N}$ without modifying the coset. We label each coset using the representative $S$ that is as close as possible to the identity rotation. We call the set of such representatives the fundamental Voronoi cell, denoting it by $\mathrm{F}_{\mathrm{SO}_{3} / \mathrm{Z}_{N}}$ (see Appendix B). It is shown in blue in Fig. 2(b) for $N=6$.

More generally, the $N$ images of $\mathrm{F}_{\mathrm{SO}_{3} / \mathrm{Z}_{N}}$ under the action of passive rotations $R \in \mathrm{Z}_{N}$ are called Voronoi cells of $R$. These cells are disjoint, and together they cover $\mathrm{SO}_{3}$ in what is known as a Voronoi tiling. The six cells for $N=6$ are shown in groups of three in Fig. 3(c), right and left. Voronoi tilings exist for all discrete subgroups $\mathrm{H} \subset \mathrm{SO}_{3}$; fundamental cells $\mathrm{F}_{\mathrm{SO}_{3} / \mathrm{H}}$ for various $\mathrm{H}$ are bounded in orange and blue in Fig. 4.

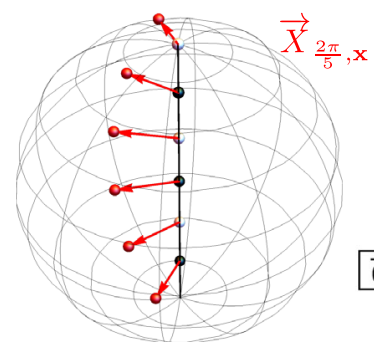

(a) Position shift

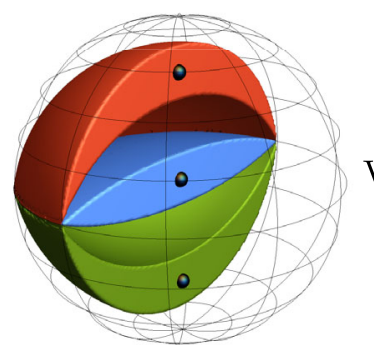

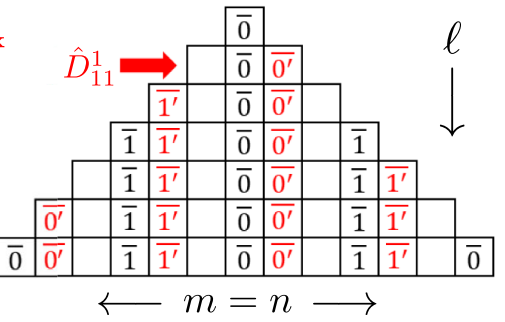

(b) Momentum kick

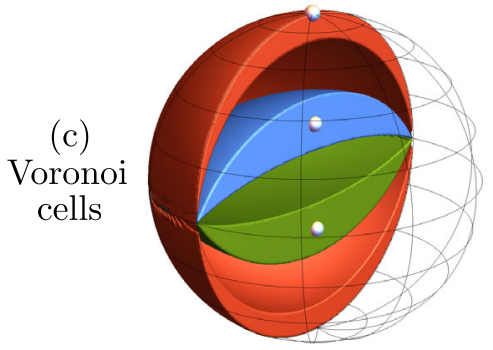

FIG. 3. Error and recovery. (a) Effect of a representative position shift on the code word group elements. The group $\mathrm{SO}_{3}$ is represented as a 3-ball with antipodal points identified. The three group elements which are superposed in the code word $|\overline{0}\rangle(|\overline{1}\rangle)(45)$ are indicated as black (white) balls. Upon the indicated representative position shift, each group element is mapped to a group element corresponding to the red ball linked by a red arrow. (b) Sketch of the ||$\left._{m m}^{\ell}\right\rangle$ angular-momentum pyramid for $0 \leq|m| \leq \ell$, where nonzero components of the logical- $X$ eigenstates $|\overline{0}\rangle_{X}$ and $|\overline{1}\rangle_{X}$ (49) are marked in black for the case $N=3$. Under the momentum kick $\hat{D}_{11}^{1}$ (54), these code words are mapped to error states $\left|\overline{0^{\prime}}\right\rangle_{X}$ and $\left|\overline{1^{\prime}}\right\rangle_{X}$, whose components are marked in red. (c) Voronoi cells of group elements corresponding to code word $|\overline{0}\rangle$ (left) and $|\overline{1}\rangle$ (right) are indicated as a red, blue, or green region.

\section{Code words}

As in Sec. IV A, code basis states are associated with the elements of the coset space $\mathrm{Z}_{d N} / \mathrm{Z}_{N}$, where $\mathrm{Z}_{d N}$ is a larger group of rotations about the $\mathbf{z}$ axis that contains $Z_{N}$. Here, for simplicity, we assume that $d=2$, but the generalization to other values of $d$ is straightforward. Then, the code words are

$$
\begin{aligned}
& |\overline{0}\rangle=\frac{1}{\sqrt{N}} \sum_{h \in Z_{N}}\left|\frac{2 \pi}{N} h, \mathbf{z}\right\rangle, \\
& |\overline{1}\rangle=\frac{1}{\sqrt{N}} \sum_{h \in Z_{N}}\left|\frac{2 \pi}{N} h+\frac{\pi}{N}, \mathbf{z}\right\rangle .
\end{aligned}
$$

The state $|\overline{0}\rangle$ is the uniform superposition of elements of $Z_{N}$, while $|\overline{1}\rangle$ is the uniform superposition of the elements of the nontrivial coset of $Z_{N}$ in $Z_{2 N}$, elements displaced from $\mathrm{Z}_{N}$ by $R_{(\pi / N), \mathbf{z}}$, the $\pi / N$ rotation about the $\mathbf{z}$ axis. In terms of the partially Fourier-transformed basis Eq. (44), we may express the code words as 


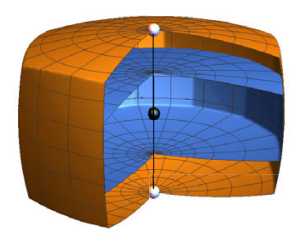

(a) $\mathrm{D}_{3} \subset \mathrm{D}_{6}$ on $\mathrm{SO}_{3}$

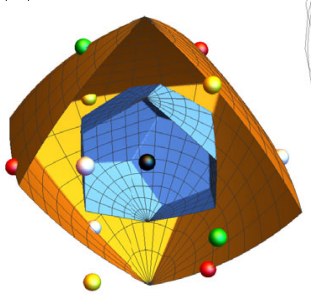

(c) $\mathrm{T} \subset \mathrm{I}$ on $\mathrm{SO}_{3}$

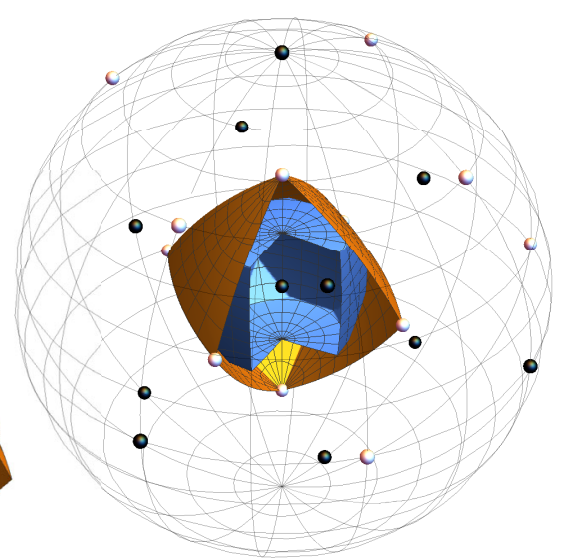

(b) $\mathrm{T} \subset \mathrm{O}$ on $\mathrm{SO}_{3}$

FIG. 4. Non-Abelian subgroup codes. (a) Sketch of the prism spaces $\mathrm{SO}_{3} / \mathrm{D}_{3}$ (orange), $\mathrm{SO}_{3} / \mathrm{D}_{6}$ (blue), and group elements representing the two logical code words within $\mathrm{SO}_{3} / \mathrm{D}_{3}$ for the $\mathrm{D}_{3} \subset \mathrm{D}_{6}$ dihedral code from Sec. V D. Similar sketches for the quotient spaces (see Table I) and code words of (b) the $\mathrm{T} \subset \mathrm{O}$ and (c) the $\mathrm{T} \subset \mathrm{I}$ codes from Sec. VE.

$$
|\overline{0}\rangle=\left|\mathrm{Z}_{N} ; 0\right\rangle \quad \text { and } \quad|\overline{1}\rangle=\left|R_{(\pi / N), \mathbf{z}} \mathrm{Z}_{N} ; 0\right\rangle .
$$

Using Eq. (34), we can also express these code words in the angular-momentum eigenstate basis. Since $D_{m n}^{\ell}(R)$ is a diagonal matrix for any rotation $R$ about the $\mathbf{z}$ axis, [145],

$$
D_{m n}^{\ell}(\omega, \mathbf{z})=\delta_{m n} e^{i m \omega}
$$

we easily compute (for $r \in\{0,1\}$ )

$$
\left.|\bar{r}\rangle=\left.\sum_{\ell \geq 0} \sqrt{\frac{N(2 \ell+1)}{8 \pi^{2}}} \sum_{|p N| \leq \ell}(-1)^{p r}\right|_{p N, p N} ^{\ell}\right\rangle .
$$

We see that, when expanded in the $\left|{ }_{m n}\right\rangle$ basis, the only states that occur with nonzero coefficients are those for which $m=n$ is an integer multiple of $N$. This property ensures that the code words are well protected against sufficiently small angular-momentum kicks.

It is also useful to express the code words in the logical- $X$ basis, defining $|\bar{r}\rangle_{X}=(1 / \sqrt{2})\left[|\overline{0}\rangle+(-1)^{r}|\overline{1}\rangle\right]$ and finding

$$
\begin{aligned}
|\overline{0}\rangle_{X} & \left.=\sum_{\ell \geq 0} \sqrt{\frac{N(2 \ell+1)}{4 \pi^{2}}} \sum_{|2 p N| \leq \ell}||_{2 p N, 2 p N}^{\ell}\right\rangle, \\
|\overline{1}\rangle_{X} & \left.=\sum_{\ell \geq N} \sqrt{\frac{N(2 \ell+1)}{4 \pi^{2}}} \sum_{|(2 p+1) N| \leq \ell}||_{(2 p+1) N,(2 p+1) N}^{\ell}\right\rangle .
\end{aligned}
$$

The code word $|\overline{0}\rangle_{X}$, expanded in the $\left.\left.\right|_{m n} ^{\ell}\right\rangle$ basis, includes only basis states in which $m=n$ is an even multiple of $N$, and the code word $|\overline{1}\rangle_{X}$ includes only basis states with $m$ an

odd multiple of $N$. The ||$\left._{m n}^{\ell}\right\rangle$ 's which occur with nonzero coefficients are indicated schematically in Fig. 2(b) for $N=3$, with black squares indicating basis states in the expansion of $|\overline{0}\rangle_{X}$ and white squares indicating basis states in the expansion of $|\overline{1}\rangle_{X}$.

\section{Position shifts}

We correct an error of the form Eq. (43) in two steps. In the first step, we diagnose and reverse the shift in the position basis $\vec{X}_{S}:|R\rangle \rightarrow|S R\rangle$. After the position shift is corrected, we can proceed to correct the momentum kick $\hat{D}_{m n}^{\ell}$.

For any coset space $\mathrm{G} / \mathrm{H}$, we can label the cosets using coset representatives chosen from the fundamental Voronoi cell $\mathrm{F}_{\mathrm{G} / \mathrm{H}}$. Then, the action of $\mathrm{G}$ on the cosets is described by the induced representation [153-155]

$$
\vec{X}_{R}|S \mathrm{H} ; 0\rangle=|R S T \mathrm{H} ; 0\rangle,
$$

where $T$ is a compensating element of $\mathrm{H}$ chosen to ensure that $R S T \in \mathrm{F}_{\mathrm{G} / \mathrm{H}}$. (If $R S \in \mathrm{F}_{\mathrm{G} / \mathrm{H}}$, then no compensating element of $\mathrm{H}$ is needed.)

The effect of a representative rotation on the group elements making up the code words is shown in Fig. 3(a). For correcting position shifts acting on the code words, the relevant coset space is $\mathrm{SO}_{3} / \mathrm{Z}_{2 N}$. We quotient out $\mathrm{Z}_{2 N}$ rather than $\mathrm{Z}_{N}$, because both $|\overline{0}\rangle$ and $|\overline{1}\rangle$ are superpositions of elements of $\mathrm{Z}_{2 N}$; hence, an element of $\mathrm{SO}_{3} / \mathrm{Z}_{2 N}$ characterizes the shift away from the code space induced by $\vec{X}_{R}$ in Eq. (50), without revealing any information that distinguishes $|\overline{0}\rangle$ from $|\overline{1}\rangle$. We divide the basis elements $\left\{\left|S \mathrm{Z}_{N} ; \lambda\right\rangle\right\}(44)$ into two disjoint subsets, where each subset is parametrized by an element of $\mathrm{F}_{\mathrm{SO}_{3} / \mathrm{Z}_{2 N}}$ rather than an element of $\mathrm{F}_{\mathrm{SO}_{3} / \mathrm{Z}_{N}}$, as follows:

$$
\begin{aligned}
\left|\tilde{S} \mathrm{Z}_{N} ; \lambda\right\rangle & =\vec{X}_{\tilde{S}}\left|\mathrm{Z}_{N} ; \lambda\right\rangle \quad \text { and } \\
\left|\tilde{S} R_{(\pi / N), \mathbf{z}} \mathrm{Z}_{N} ; \lambda\right\rangle & =\vec{X}_{\tilde{S}}\left|R_{(\pi / N), \mathbf{z}} \mathrm{Z}_{N} ; \lambda\right\rangle,
\end{aligned}
$$

where $\lambda \in\{0,1, \ldots, N-1\}$ as before, but now $\tilde{S} \in \mathrm{F}_{\mathrm{SO}_{3} / \mathrm{Z}_{2 N}}$. Since each element of $S \in \mathrm{F}_{\mathrm{SO}_{3} / \mathrm{Z}_{N}}$ can be uniquely expressed as either $S=\tilde{S}$ or $S=\tilde{S} R_{(\pi / N), \mathbf{z}}$, for $\tilde{S} \in \mathrm{F}_{\mathrm{SO}_{3} / \mathrm{Z}_{2 N}}$, this basis is the same basis as described earlier, just with a different labeling than before. The first set is the set of all states obtained by acting with a rotation $\tilde{S} \in \mathrm{F}_{\mathrm{SO}_{3} / \mathrm{F}_{2 N}}$ on the logical zero code word; the second set is obtained similarly from logical one. (If we wish to construct a $d$ dimensional code space, we would divide the basis into $d$ disjoint subsets, with each subset parametrized by an element of $\mathrm{F}_{\mathrm{SO}_{3} / \mathrm{Z}_{d N}}$.)

To diagnose the error, we measure the value of $\tilde{S}$ and then apply $\vec{X}_{\tilde{S}}^{\dagger}$ to attempt to correct the effect of the position shift. If the actual shift error is $\vec{X}_{R}$, where $R$ is contained in 
the $\mathrm{F}_{\mathrm{SO}_{3} / \mathrm{Z}_{2 N}}$, this recovery procedure successfully corrects the position-shift error, mapping

$$
\begin{aligned}
\vec{X}_{R}\left|\mathrm{Z}_{N} ; \lambda\right\rangle & \rightarrow\left|\mathrm{Z}_{N} ; \lambda\right\rangle, \\
\vec{X}_{R}\left|R_{(\pi / N), \mathbf{z}} \mathrm{Z}_{N} ; \lambda\right\rangle & \rightarrow\left|R_{(\pi / N), \mathbf{z}} \mathrm{Z}_{N} ; \lambda\right\rangle .
\end{aligned}
$$

A subsequent momentum-kick correction, described below, then completes the recovery, correctly mapping the corrupted states back to their respective code words (46). However, if $R$ is not contained in $\mathrm{F}_{\mathrm{SO}_{3} / Z_{2 N}}$, then there may be an uncorrected logical error which interchanges $\left|Z_{N} ; \lambda\right\rangle$ and $\left|R_{(\pi / N), \mathrm{z}} \mathrm{Z}_{N} ; \lambda\right\rangle$ on the right-hand side of Eq. (52).

Thus, the code protects against any position-shift error $\vec{X}_{R}$ for $R \in \mathrm{F}_{\mathrm{SO}_{3} / \mathrm{Z}_{2 N}}$. This set of correctable rotations is indicated by the blue region in Fig. 2(b). How large an angular rotation can be tolerated depends on the axis of rotation and can be determined by analyzing the geometry of the fundamental cell $\mathrm{F}_{\mathrm{SO}_{3} / \mathrm{Z}_{2 N}}$ (see Appendix B). We find that a rotation by angle $\omega$ about an axis with polar angle $\Theta$ is contained within $\mathrm{F}_{\mathrm{SO}_{3} / \mathrm{Z}_{2 N}}$ for

$$
|\omega|<\omega_{\max }(\Theta) \equiv\left|2 \cot ^{-1}\left(\cos \Theta \cot \frac{\pi}{4 N}\right)\right|
$$

The maximum correctable rotation angle $\omega_{\max }(\Theta)$ is smallest for rotations about the $\mathbf{z}$ axis, where $\omega_{\max }(0)=$ $(\pi / 2 N)$, as for the planar-rotor code discussed in Sec. IVA. The largest correctable rotation angles occur when the rotation axis is in the equatorial plane, where $\omega_{\max }(\pi / 2)=\pi$. Thus, any rotation about such an axis is correctable, unless the rotation angle is precisely $\pi$. The relative volume occupied by correctable rotations in $\mathrm{SO}_{3}$ is $1 / 2 N$.

\section{Momentum kicks}

We have now described how to correct the position shift $\vec{X}_{R}$ in Eq. (43). Next, we need to understand how to contend with a momentum kick

$$
\hat{D}_{m n}^{\ell} \equiv \int_{\mathrm{SO}_{3}} d R|R\rangle D_{m n}^{\ell}(R)\langle R|
$$

acting on the code space.

We can compute the action of $\hat{D}_{m n}^{\ell}$ on the code words using Eq. (47), finding

$$
\begin{aligned}
& \hat{D}_{m n}^{\ell}|\overline{0}\rangle=\delta_{m n}\left|\mathrm{Z}_{N} ; \lambda=m(\bmod N)\right\rangle, \\
& \hat{D}_{m n}^{\ell}|\overline{1}\rangle=\delta_{m n} e^{i(\pi / N) m}\left|R_{(\pi / N), \mathbf{z}} \mathrm{Z}_{N} ; \lambda=m(\bmod N)\right\rangle .
\end{aligned}
$$

After the noise acts on the encoded state, and the positionshift error is corrected, we measure the value of $\lambda$, the syndrome for the momentum-shift error. The key thing to notice is that, while $\lambda$ determines the value of $m(\bmod N)$, the code-word-dependent phase $e^{i(\pi / N) m}$ in Eq. (55) depends on the value of $m(\bmod 2 N)$. In fact, for any value of $\ell \geq N$, the operator $\hat{D}_{N N}^{\ell}$ is a nontrivial logical operator that preserves the code space and flips the relative phase of $|\overline{0}\rangle$ and $|\overline{1}\rangle$.

Once the value of $\lambda$ is known, we attempt recovery by applying the unitary operator $U_{m}$ with the action

$$
\begin{aligned}
U_{m}: & \left|\mathrm{Z}_{N} ; m\right\rangle \rightarrow\left|\mathrm{Z}_{N} ; 0\right\rangle, \\
& \left|R_{(\pi / N), \mathbf{z}} \mathrm{Z}_{N} ; m\right\rangle \rightarrow e^{-i(\pi / N) m}\left|R_{(\pi / N), \mathbf{z}} \mathrm{Z}_{N} ; 0\right\rangle,
\end{aligned}
$$

where $m$ is chosen to be the integer with a minimal absolute value such that $m=\lambda(\bmod N)$. For example, we can choose $U_{m}$ to have the same action as $\hat{D}_{m m}^{N \dagger}$ on the position eigenstates $\left|R_{\omega, \mathbf{z}}\right\rangle$, namely,

$$
U_{m}\left|R_{\omega, \mathbf{z}}\right\rangle=e^{-i m \omega}\left|R_{\omega, \mathbf{z}}\right\rangle .
$$

How $U_{m}$ acts on $|R\rangle$ when $R$ is not a rotation about the $\mathbf{z}$ axis can be chosen arbitrarily. We note, though, that this extended operator can be diagonal in the $\{|R\rangle\}$ basis. That is, the recovery operation after a momentum kick can be a phase shift that depends on the orientation of the rotor and has the action Eq. (57) when the rotor's orientation differs from the standard reference orientation by a rotation about the $\mathbf{z}$ axis.

Notice that $m(\bmod 2 N)$ is unambiguously determined by $\lambda=m(\bmod N)$ for any $m$ satisfying $|m|<N / 2$. Therefore, the damage to the code words caused by the action of $\hat{D}_{m n}^{\ell}$ is corrected successfully when $|m|<N / 2$. Since $|m| \leq \ell$, we conclude that the code protects against any momentum kick $\hat{D}_{m n}^{\ell}$ such that

$$
\ell<N / 2
$$

For $\ell \geq m>N / 2$, however, a logical error may occur.

It is also instructive to consider how the momentum kick $\hat{D}_{m n}^{\ell}$ acts on the basis of angular-momentum eigenstates. We observe that

$$
\begin{aligned}
& \left\langle\left.{ }_{M N}^{L}\left|\hat{D}_{m n}^{\ell}\right|\right|_{m^{\prime} n^{\prime}} ^{\ell^{\prime}}\right\rangle=\int_{\mathrm{SO}_{3}} d R\left\langle_{M N}^{L} \mid R\right\rangle D_{m n}^{\ell}(R)\left\langle\left. R\right|_{m^{\prime} n^{\prime}} ^{\ell^{\prime}}\right\rangle \\
& =\frac{\sqrt{(2 L+1)\left(2 \ell^{\prime}+1\right)}}{8 \pi^{2}} \int_{\mathrm{SO}_{3}} d R D_{M N}^{L \star}(R) D_{m n}^{\ell}(R) D_{m^{\prime} n^{\prime}}^{\ell^{\prime}}(R) .
\end{aligned}
$$

The group integral (59) can be expressed in terms of Clebsch-Gordan coefficients (see Table IV G). For our purposes, what is noteworthy is that selection rules for addition of angular momenta require the integral to vanish unless 
$\left|\ell^{\prime}-\ell\right| \leq L \leq \ell^{\prime}+\ell, \quad M=m^{\prime}+m, \quad N=n^{\prime}+n$.

As indicated in Eq. (48), if the code words are expanded in the $\left.\left\{||_{m^{\prime} n^{\prime}}^{\ell^{\prime}}\right\rangle\right\}$ basis, then $m^{\prime}=n^{\prime}$ is an integer multiple of $N$ for all states that occur with nonzero coefficients. For $\ell<N / 2$, the momentum kick $\hat{D}_{m n}^{\ell}$ changes the value of $m^{\prime}$ and $n^{\prime}$ by less than half the spacing between successive multiples of $N$. Therefore, these shifts in $m^{\prime}$ and $n^{\prime}$ can be unambiguously identified and corrected. However, it is simpler to understand how the recovery procedure works in detail using the expansion of the code words in the position basis $\{|R\rangle\}$ [as in Eq. (56)] rather than the angularmomentum basis (detailed in Appendix C).

The effect of an angular-momentum kick $\hat{D}_{1,1}^{1}$ is visualized in Fig. 3(b) for the case $N=3$. Recalling Eq. (55), this kick shifts the code space to a subspace with $\lambda=1$, and $\hat{D}_{-1,-1}^{1}$ shifts the code space to a subspace with $\lambda=2$. In either case, measuring $\lambda$ points to a unique error with $\ell \leq 1$ which can then be corrected. However, $\hat{D}_{2,2}^{2}$ also maps the code space to the same subspace with $\lambda=2$ as $\hat{D}_{-1,-1}^{1}$, imparting a different code-word-dependent phase; according to Eq. (55), the code word $|\overline{1}\rangle$ acquires the relative phase $\exp [-i(\pi / 3)]$ when $\hat{D}_{-1,-1}^{1}$ acts on the code space and the relative phase $\exp [i(2 \pi / 3)]$ when $\hat{D}_{2,2}^{2}$ acts on the code space. Therefore, if the $\hat{D}_{2,2}^{2}$ error occurs and is misdiagnosed as a $\hat{D}_{-1,-1}^{1}$ error, a nontrivial logical error results when recovery is attempted.

\section{Logical operators}

The unitary active rotation $\vec{X}_{(\pi / N), \mathbf{z}}$, acting on the code words in Eq. (45), has the effect of interchanging $|\overline{0}\rangle$ and $|\overline{1}\rangle$. It can be regarded as the logical Pauli operator $\bar{X}$ acting on the code space. This operation can similarly be performed by the passive rotation $\overleftarrow{X}_{(\pi / N), \mathbf{z}}$ [since the code words (45) consist of position states forming an Abelian group]. In other words, we can rotate the molecular frame or the lab frame to perform this operation. Active and passive rotations always commute: $\vec{X}_{R} \bar{X}_{S}=\bar{X}_{S} \vec{X}_{R}$. We use this fact to infer the momentum-kick syndrome $\lambda$ using passive rotations, without interfering with position shifts $\vec{X}_{R}$.

We have already noted that $\hat{D}_{N N}^{\ell}$ (for any $\ell \geq N$ ), acting on the code words, preserves $|\overline{0}\rangle$ and flips the phase of $|\overline{1}\rangle$. Thus, its action on the code space is equivalent to the logical Pauli operator $\bar{Z}$. However, $\hat{D}_{N N}^{\ell}$ is not unitary as an operator acting on the full Hilbert space of the rotor. Why is $\hat{D}_{N N}^{\ell}$ not unitary? Recall that $\hat{D}_{m n}^{\ell}$ is diagonal in the $\{|R\rangle\}$ basis, with eigenvalues $\left\{D_{m n}^{\ell}(R)\right\}$. The trouble is that, for rotations that are not about the $\mathbf{z}$ axis, $\left\{D_{m n}^{\ell}(R)\right\}$ does not have modulus 1 and, therefore, cannot be an eigenvalue of a unitary operator. Specifically, if we parametrize $R_{\alpha \beta \gamma}$ using
Euler angles in the $Z Y Z$ convention, where $\alpha \in[0,2 \pi)$, $\beta \in[0, \pi]$, and $\gamma \in[0,2 \pi)$,

$$
D_{N N}^{N}(\alpha, \beta, \gamma)=e^{i N(\alpha+\gamma)} \cos ^{2 N}(\beta / 2),
$$

which has modulus less than 1 for nonzero $\beta$.

To formulate an implementation of $\bar{Z}$ that is achievable in the laboratory, we should find a unitary extension of its logical action to the full Hilbert space. As for the recovery operation described earlier, we can choose this logical $\bar{Z}$ to be a phase shift that depends appropriately on the orientation of the rotor.

One way to produce logical gates involves turning on the Hamiltonian $\hat{D}_{N N}^{N}+$ H.c. to perform a logical $Z$-axis rotation with an angle proportional to the time the Hamiltonian is turned on. This rotation provides unitary logical $Z$ gates, and analogous two-qubit $Z Z$ gates can be performed via the Hamiltonian $\hat{D}_{N N}^{N} \otimes \hat{D}_{N N}^{N}+$ H.c. However, such gates are subject to over- or underrotation errors.

\section{Check operators}

Once we have operators whose action on the code space matches that of the logical Pauli operators $\bar{X}$ and $\bar{Z}$, we can square these operators to define the check operators for the code. Then, the code space can be said to be the simultaneous eigenspace with eigenvalue 1 of these operators.

Recall that $\bar{X}$ is a position shift defined as either left or right multiplication by $R_{(\pi / N), \mathbf{z}}$, corresponding to either an active or a passive rotation, respectively. We choose our " $X$-type" stabilizer to be a passive rotation, yielding the unitary operator

$$
\hat{S}_{X}=\left(\overleftarrow{X}_{(\pi / N), \mathbf{z}}\right)^{2}=\overleftarrow{X}_{(2 \pi / N), \mathbf{z}}
$$

The condition $\hat{S}_{X}=1$ requires the code words to be invariant under a position shift by $R_{(2 \pi / N), \mathbf{z}}$. The additional benefit of using a passive rotation is that all of the partial Fourier-transformed states (44) are eigenstates of $\hat{S}_{X}$ :

$$
\overleftarrow{X}_{(2 \pi / N), \mathbf{z}}\left|S Z_{N} ; \lambda\right\rangle=e^{i(2 \pi / N) \lambda}\left|S Z_{N} ; \lambda\right\rangle
$$

This way, the syndrome $\lambda$ can be extracted via a projective measurement onto eigenspaces of $\hat{S}_{X}$.

As we note, $\hat{D}_{N N}^{N}$ is not unitary, but nevertheless we may square it to obtain a (nonunitary) $Z$-type check operator

$$
\begin{aligned}
\hat{S}_{Z} & =\left(\hat{D}_{N, N}^{N}\right)^{2}=\hat{D}_{2 N, 2 N}^{2 N} \\
& =\int \sin \beta d \alpha d \beta d \gamma\left|R_{\alpha \beta \gamma}\right\rangle e^{i 2 N(\alpha+\gamma)} \cos ^{4 N}(\beta / 2)\left\langle R_{\alpha \beta \gamma}\right| .
\end{aligned}
$$


The eigenspace of $\hat{S}_{Z}$ with eigenvalue 1 contains rotations about the $\mathbf{z}$ axis $(\beta=0)$, by angle $\omega=\alpha+\gamma=(\pi / N) h$, where $h \in\{0,1, \ldots, 2 N-1\}$. The only states that satisfy these conditions and that are also invariant under $\hat{S}_{X}$ are the states in the code space.

To check that $\hat{S}_{X}$ and $\hat{S}_{Z}$ are really commuting operators, we observe that

$$
\begin{aligned}
\overleftarrow{X}_{S} \hat{D}_{m n}^{\ell} \bar{X}_{S}^{\dagger} & =\int_{\mathrm{SO}_{3}} d R\left|R S^{-1}\right\rangle D_{m n}^{\ell}(R)\left\langle R S^{-1}\right| \\
& =\int_{\mathrm{SO}_{3}} d R|R\rangle D_{m n}^{\ell}(R S)\langle R|,
\end{aligned}
$$

where we use the invariance of the Haar measure to obtain the second equality. Furthermore, if $S$ is a rotation about the $\mathbf{z}$ axis, then, recalling the $Z Y Z$ Euler-angle convention used here, right multiplication by $S$ merely changes the third angle:

$$
R_{\alpha, \beta, \gamma} R_{\omega, 0,0}=R_{\alpha, \beta, \gamma+\omega} .
$$

Using this result and Eq. (61),

$$
D_{N N}^{N}\left(\alpha, \beta, \gamma+\frac{\pi}{N}\right)=-D_{N N}^{N}(\alpha, \beta, \gamma)
$$

we see that $\hat{D}_{N N}^{N}$ and $\overleftarrow{X}_{(\pi / N), \mathbf{z}}$ anticommute, not only acting on the code space, but also acting on the whole Hilbert space of the rotor. Correspondingly, $\hat{S}_{Z}=\left(\hat{D}_{N N}^{N}\right)^{2}$ and $\hat{S}_{X}=\left(\overleftarrow{X}_{(\pi / N), \mathbf{z}}\right)^{2}$ commute and, thus, can be simultaneously diagonalized.

\section{B. Measurement and initialization}

Generalizing our discussion for the $\mathrm{U}_{1}$ rotor from Sec. IV B, we outline procedures for extracting the momentum $\left(\lambda \in \mathrm{Z}_{N}\right)$ and position $\left(\tilde{S} \in \mathrm{F}_{\mathrm{SO}_{3} / \mathrm{Z}_{2 N}}\right)$ shift values using ancilla systems. These procedures also allow us to perform logical state initialization.

\section{Momentum syndromes}

There are $N$ different possible values of $\lambda$, so a one-shot measurement requires an ancilla with at least $N$ orthogonal states. We use a qunit ancilla with $Z$ eigenstates $\left|h_{z}\right\rangle, X$ eigenstates $\left|h_{x}\right\rangle\left(h \in \mathrm{Z}_{N}\right)$, and Pauli operator $\mathcal{X}$ satisfying $\mathcal{X}\left|h_{z}\right\rangle=\left|h+1_{z}\right\rangle$ (modulo $N$ ).

To measure $\lambda$, we initialize the qunit in $\left|0_{z}\right\rangle$ and entangle it with the rigid rotor by applying the gate

$$
\begin{aligned}
\mathrm{CPHS} & =\sum_{\ell \leq 0} \sum_{|m|,|n| \leq \ell}\left|{ }_{m n}^{\ell}\right\rangle\left\langle{ }_{m n}^{\ell}\right| \otimes \mathcal{X}^{n} \\
& =\sum_{h \in Z_{N}} \overleftarrow{X}_{(2 \pi / N) h, \mathbf{z}}^{\dagger} \otimes\left|h_{x}\right\rangle\left\langle h_{x}\right|,
\end{aligned}
$$

where the second line is obtained using Eqs. (37b) and (47). This gate shifts the "position" of the qunit by $n$, conditioned on the rotor having angular-momentum $\mathbf{z}$ component $n$ in the rotor frame. Applying this result to a partially Fouriertransformed basis state (44) and using Eq. (63) yields

$$
\mathrm{CPHS}\left|S \mathrm{Z}_{N} ; \lambda\right\rangle \otimes\left|0_{z}\right\rangle=\left|S \mathrm{Z}_{N} ; \lambda\right\rangle \otimes\left|\lambda_{z}\right\rangle .
$$

The value of $\lambda$ is thus mapped onto the ancilla, and a subsequent $Z$-basis measurement of the ancilla allows us to extract $\lambda$.

\section{Position syndromes}

To diagnose position shifts without disturbing the logical information, we have to use an ancilla to measure the

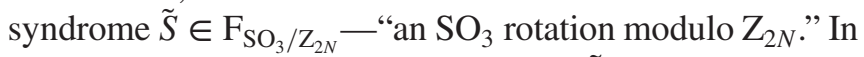
order to perfectly resolve all possible $\tilde{S}$ in one shot, the ancilla needs to admit an orthonormal set of position states

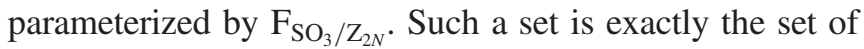
orientations of a $Z_{2 N}$-symmetric rigid body (see Sec. III C). However, coupling an asymmetric molecule to a symmetric one is difficult. Below, we show how to approximate the required position states using generalized spin-coherent states of a finite-dimensional spin (see Sec. III B).

We use a spin- $L$ ancilla $\{|L\rangle,|s| \leq L\}$, which admits an irrep of $\mathrm{SO}_{3}$ with corresponding rotation matrices $D^{L}(R)$. In order to "mod out" the $\mathrm{Z}_{2 N}$ rotation, we initialize the ancilla in any state $\left|Z_{2 N}\right\rangle$ whose maximal invariant subgroup is $\mathrm{Z}_{2 N}$, i.e.,

$$
\left|\left\langle\mathrm{Z}_{2 N}\left|D^{L}(T)\right| \mathrm{Z}_{2 N}\right\rangle\right| \begin{cases}=1 & T \in \mathrm{Z}_{2 N}, \\ <1 & \text { otherwise. }\end{cases}
$$

Such states exist for any $L \geq N$ (Table 10.1 in Ref. [156]); (see also Refs. $[107,157]$ ). For example, the $L=N$ family of states $\left.\left.\left.\cos \eta\right|_{N} ^{N}\right\rangle+\left.\sin \eta\right|_{-N} ^{N}\right\rangle$ satisfies the above for any $\eta \in(0, \pi / 4)$.

Any rotation $R \in \mathrm{SO}_{3}$ can be written as $R=\tilde{S} T$, with $\tilde{S} \in \mathrm{F}_{\mathrm{SO}_{3} / \mathrm{Z}_{2 N}}$ and $T \in \mathrm{Z}_{2 N}$. Therefore, applying rotations to $\left|Z_{2 N}\right\rangle$ and using Eq. (35) yields the following set of generalized spin-coherent states $|\tilde{S}\rangle_{\mathrm{Z}_{2 N}}$ :

$$
\begin{aligned}
D^{L}(R)\left|\mathrm{Z}_{2 N}\right\rangle & =D^{L}(\tilde{S}) D^{L}(T)\left|\mathrm{Z}_{2 N}\right\rangle \\
& \propto D^{L}(\tilde{S})\left|\mathrm{Z}_{2 N}\right\rangle \equiv|\tilde{S}\rangle_{\mathrm{Z}_{2 N}}
\end{aligned}
$$

parameterized by $\tilde{S} \in \mathrm{F}_{\mathrm{SO}_{3} / \mathrm{Z}_{2 N}}$.

To map the syndrome onto the ancilla, we use the conditional rotation [cf. Eq. (4) in Ref. [158] ]

$$
\mathrm{CROT}=\int_{\mathrm{SO}_{3}} d R|R\rangle\langle R| \otimes D^{L}(R) .
$$

This gate applies a rotation $D(R)$ on the ancilla, conditioned on the rotor being in the state $|R\rangle$. When applied to 
the specified ancillary state, this gate maps $\tilde{S} \in \mathrm{F}_{\mathrm{SO}_{3} / \mathrm{Z}_{2 N}}$ onto the ancilla while ignoring the logical state index. Applying it to the two sets of basis states from Eq. (51), we have

$$
\begin{aligned}
\mathrm{CROT}\left|\tilde{S} \mathrm{Z}_{N} ; \lambda\right\rangle \otimes\left|\mathrm{Z}_{2 N}\right\rangle & =\left|\tilde{S} \mathrm{Z}_{N} ; \lambda\right\rangle \otimes|\tilde{S}\rangle_{\mathrm{Z}_{2 N}}, \\
\mathrm{CROT}\left|\tilde{S} R_{(\pi / N), \mathrm{z}} \mathrm{Z}_{N} ; \lambda\right\rangle \otimes\left|\mathrm{Z}_{2 N}\right\rangle & =\left|\tilde{S} R_{(\pi / N), \mathrm{z}} \mathrm{Z}_{N} ; \lambda\right\rangle \otimes|\tilde{S}\rangle_{\mathrm{Z}_{2 N}}
\end{aligned}
$$

Unfortunately, a projective measurement in the overcomplete $|\tilde{S}\rangle_{\mathrm{Z}_{2 N}}$ set of the states does not yield $\tilde{S}$ exactly, since the spin-coherent states are not orthogonal for any finite $L$ (see Sec. II. 3 in Ref. [99]). However, they approach orthogonality in the limit $L \rightarrow \infty$, meaning that a sufficiently large spin should be able to resolve points in $\mathrm{F}_{\mathrm{SO}_{3} / \mathrm{Z}_{2 N}}$ to desired accuracy.

\section{Initialization}

The CROT gate can also be used to initialize in the logical- $X$ state $|\overline{0}\rangle_{X} \propto \sum_{T \in Z_{2 N}}|T\rangle$ (49). Say the rotor instead starts in the lowest-momentum state $\left.\left.\right|_{00} ^{0}\right\rangle$ - a state outside of the code space. Then, application of the gate yields (up to normalization)

$\left.\mathrm{CROT}||_{00}^{0}\right\rangle \otimes\left|\mathrm{Z}_{2 N}\right\rangle \propto \int_{\mathrm{F}_{\mathrm{SO}_{3} / \mathrm{Z}_{2 N}}} d \tilde{S} \sum_{T \in \mathrm{Z}_{2 N}}|\tilde{S} T\rangle \otimes|\tilde{S}\rangle_{\mathrm{Z}_{2 N}}$.

A projective measurement obtaining some $\tilde{S}$ followed by a rotation $\vec{X}_{\tilde{S}}^{\dagger}$ yields the desired logical state.

\section{Normalizable code words}

Our logical code words are not normalizable and, therefore, unphysical. To obtain normalizable states, we may regulate the sum over the angular momentum $\ell$ in Eq. (48) by introducing a broad envelope function which decays sufficiently rapidly for large $\ell$. In position space, this regulation corresponds to replacing the position eigenstate $|\omega, \mathbf{z}\rangle$ by a sharply peaked normalizable wave packet which approximates $|\omega, \mathbf{z}\rangle$. The protection against position shifts is mildly impaired due to this spreading of the code words in position space. On the other hand, these approximate code words still have support on angular-momentum states such that $\ell$ is an integer multiple of $N$, and, therefore, the code continues to detect momentum-kick operators $\hat{D}_{m m}^{\ell}$ with $\ell<N$ and $|m|>0$. However, the kicks $\hat{D}_{00}^{\ell}$ no longer leave the code words invariant [as in Eq. (55)], inducing a slight $\ell$-dependent distortion that can cause a logical error.

The oscillator GKP codes can be regulated [1] by applying the damping function $\exp \left(-\frac{1}{2} \Delta^{2} \hat{n}\right)$ to the ideal code words, where $\hat{n}$ is the number operator and $\Delta>0$ is the damping strength [see Eq. (7.12) in Refs. [3] and
Ref. [4]]. For molecular codes with configuration space $\mathrm{SO}_{3}$, we analogously use the damping function $\exp \left(-\frac{1}{2} \Delta^{2} \hat{L}^{2}\right)$, where $\hat{L}^{2}$ is the total angular-momentum operator which satisfies

$$
\left.\hat{L}^{2}\left|{ }_{m n}^{\ell}\right\rangle=\left.\ell(\ell+1)\right|_{m n} ^{\ell}\right\rangle
$$

and generates orientational diffusion (see Sec. 16.6 in Ref. [154]). The approximate code words are thus (for $r \in\{0,1\})$

$$
\begin{aligned}
|\tilde{r}\rangle & =e^{-(1 / 2) \Delta^{2} \hat{L}^{2}|\bar{r}\rangle / \sqrt{\left\langle\bar{r}\left|e^{-\Delta^{2} \hat{L}^{2}}\right| \bar{r}\right\rangle}} \\
& \left.=\left.\sum_{\ell \geq 0} \sqrt{\frac{N(2 \ell+1) e^{-\Delta^{2} \ell(\ell+1)}}{8 \pi^{2}\left\langle\bar{r}\left|e^{-\Delta^{2} \hat{L}^{2}}\right| \bar{r}\right\rangle}} \sum_{|p N| \leq \ell}(-1)^{p r}\right|_{p N, p N} ^{\ell}\right\rangle .
\end{aligned}
$$

Asymmetric diffusion is also possible, yielding an additional $p$-dependent damping term.

\section{Average momentum}

The expectation value of the total angular momentum is infinite for ideal code words but finite for approximate code words. The square root of the expectation value of $\hat{L}^{2}$,

$$
\bar{\ell} \equiv\left\langle\tilde{r}\left|\hat{L}^{2}\right| \tilde{r}\right\rangle^{1 / 2} \sim \sqrt{\frac{3}{2 \Delta^{2}}-\frac{1}{4}},
$$

determines the average momentum of the approximate code words $|\tilde{r}\rangle$. In Appendix $C$, we detail the calculation that obtains the above $r$-independent result, valid in the $\Delta \rightarrow 0$ limit. While the average photon number is proportional to the total oscillator energy, energy for the rigid rotor is proportional to $\bar{l}^{2}$. For our normalizable code words, the energy scales identically with $\Delta$ as that for the normalizable GKP states [1]. The variance of $\bar{\ell}, \sigma_{\bar{\ell}}^{2}=\left\langle\tilde{r}\left|\hat{L}^{4}\right| \tilde{r}\right\rangle^{1 / 2}-\bar{\ell}^{2}=$ $O\left(\bar{\ell}^{2}\right)$, is also similar to GKP states (for the latter, photon number moments satisfy a geometric distribution [3]).

\section{Approximate correctability}

The spreading of the basis states in position space gives rise to an intrinsic error in the approximate code; the basis states $|\tilde{0}\rangle$ and $|\tilde{1}\rangle$ are imperfectly distinguishable even in the absence of noise. To quantify the probability of intrinsic memory error, we estimate $P_{\text {leak }}$ - the probability that the approximate logical zero state leaks into the union of Voronoi cells associated with the other logical code word [for $N=3$, these are the three cells in the right in Fig. 3(c)]. In Appendix $\mathrm{C}$, we find, in the $\Delta \rightarrow 0$ limit,

$$
P_{\text {leak }} \sim \csc \left(\frac{\pi}{2 N}\right) \frac{\Delta}{\sqrt{\pi}} \exp \left[-\left(\frac{\pi}{2 N \Delta}\right)^{2}\right] .
$$

The right-hand side is exponentially suppressed with $1 / \Delta^{2}$ : Similar to GKP codes [1], a gentle smearing in position 
space does not significantly affect the codes' performance. More concretely, $P_{\text {leak }} \approx 10^{-3}$ requires an average momentum of $\approx 5.4$, with $99 \%$ of the approximate code words supported on the $\ell \leq 10$ momentum subspace. A more stringent $P_{\text {leak }} \approx 10^{-6}$ corresponds to $\approx 8.1$ and requires $\ell \leq 15$ to support $99 \%$ of the code words. These are reasonable numbers for the angular momentum, with recent experiments accessing $\ell \leq 6$ [15], albeit for a diatomic molecule.

As we note, the approximate code words are supported on values of $\ell$ which are integer multiples of $N$; therefore, angular-momentum kicks with $\ell<N$ and $|m|>0$ are detectable. However, the kicks $\hat{D}_{00}^{\ell}$ slightly distort the code words, leading to the potential for a logical- $X$ error. To quantify this distortion, we calculate the matrix element $\left\langle\tilde{0}\left|\hat{D}_{00}^{\ell}\right| \tilde{1}\right\rangle$ for $\ell<N$ in Appendix C. Numerically, as $\Delta \rightarrow 0$, this element is suppressed exponentially with $1 / \Delta^{2}$ for all cases tested. An estimate of its asymptotic behavior reveals that its dependence on $\Delta$ is similar to $P_{\text {leak }}(78)$ :

$$
\left\langle\tilde{0}\left|\hat{D}_{00}^{\ell}\right| \tilde{1}\right\rangle \approx 2(2 \ell+1) \exp \left[-\left(\frac{\pi}{2 N \Delta}\right)^{2}\right] .
$$

The " $\approx$ " indicates that this asymptotic estimate is supported, to some extent, by numerical evidence.

\section{Dihedral molecular codes}

For molecular codes, $\mathrm{G}$ is the rotation group $\mathrm{SO}_{3}$, and up until now we have considered the case where $\mathrm{H}, \mathrm{K}$ are Abelian, namely, $\mathrm{H}=\mathrm{Z}_{N}$ and $\mathrm{K}=\mathrm{Z}_{d N}$ (for a code space of dimension $d$ ). We may also construct codes for which $\mathrm{K}$, and perhaps also $\mathrm{H}$, are non-Abelian subgroups of $\mathrm{SO}_{3}$. Position correction proceeds similarly as for the Abelian molecular codes: One measures values in the coset space $\mathrm{SO}_{3} / \mathrm{K}$ and applies a rotation to map back into the code space. Picking non-Abelian subgroups allows for more uniform correctable rotation sets $\mathrm{F}_{\mathrm{SO}_{3} / \mathrm{K}}$ than the saucerlike $\mathrm{F}_{\mathrm{SO}_{3} / \mathrm{Z}_{2 N}}$ from Fig. 2(b). Detectable and correctable momentum kicks $\ell$ can be read off by successive use of branching formulas, i.e., restricting $\mathrm{SO}_{3}$ irreps $D^{\ell}$ to $\mathrm{K}$ and decomposing the resulting matrix into irreps of $\mathrm{K}$, and then further restricting and decomposing into $\mathrm{H}$ irreps. Here, we describe $\mathrm{D}_{N} \subset \mathrm{D}_{2 N}$ molecular codes, where $\mathrm{D}_{N}$ is the dihedral group.

\section{Code words}

The group $\mathrm{Z}_{N}$, containing rotations about the $\mathbf{z}$ axis by angle $\omega=(2 \pi / N) h$ with $h \in\{0,1, \ldots, N-1\}$, can be extended to the dihedral group $\mathrm{D}_{N}$ by adding the $\omega=\pi$ rotation around the $\mathbf{x}$ axis. The dihedral group has $2 N$ elements, the original $N$ rotations contained in $\mathrm{Z}_{N}$ and also $N$ "reflections"-rotations by $\pi$ about $N$ equally spaced axes on the equator. In terms of Euler angles, the rotations in $\mathrm{D}_{N}$ are the elements $\{(2 \pi / N) h, 0,0\}$ of $\mathrm{SO}_{3}$, and the reflections are the elements $\{(2 \pi / N) h, \pi, 0\}$, for $h \in\{0,1, \ldots, N-1\}$.

Here, we consider an extension of the $\mathrm{Z}_{N} \subset \mathrm{Z}_{d N}$ codes to $\mathrm{D}_{N} \subset \mathrm{D}_{d N}$. For the $d=2$ case, the coset space $\mathrm{D}_{2 N} / \mathrm{D}_{N}$ contains two cosets: the trivial coset (the $\mathrm{D}_{N}$ subgroup of $\mathrm{D}_{2 N}$ ) and the nontrivial coset, which is obtained by multiplying all elements of $\mathrm{D}_{N}$ by the rotation with Euler angles $\{(\pi / N), 0,0\}$. The logical code words are

$$
\begin{aligned}
& |\overline{0}\rangle=\frac{1}{\sqrt{2 N}} \sum_{T \in \mathrm{D}_{N}}|T\rangle, \\
& |\overline{1}\rangle=\frac{1}{\sqrt{2 N}} \sum_{T \in \mathrm{D}_{N}}\left|R_{(\pi / N) 00} T\right\rangle .
\end{aligned}
$$

This code family has much in common with the $\mathrm{Z}_{N} \subset \mathrm{Z}_{2 N}$ code; in particular, it can correct momentum kicks with $\ell<N / 2$. The space of correctable position shifts-the prism space $\mathrm{SO}_{3} / \mathrm{D}_{2 N}$ [Table I and Fig. 4(a)] - gets flatter with increasing $N$. Thus, as for the $\mathrm{Z}_{N} \subset \mathrm{Z}_{2 N}$ codes, there is a trade-off: As $N$ increases, the code protects against larger momentum kicks, but at the cost of weakened performance against rotation errors.

\section{Partial Fourier transform}

For the $\mathrm{D}_{N} \subset \mathrm{D}_{2 N}$ code, the partially Fourier-transformed basis generalizing Eq. (44) consists of pairs of basis states of the form

$$
\left|S \mathrm{D}_{N} ; \mu_{\mu \nu}\right\rangle=\frac{1}{\sqrt{2 N}} \sum_{T \in \mathrm{D}_{N}} Z_{\mu \nu}^{\lambda}(T)|S T\rangle,
$$

$\left|S R_{(\pi / N) 00} \mathrm{D}_{N} ;{ }_{\mu \nu}^{\prime}\right\rangle=\frac{1}{\sqrt{2 N}} \sum_{T \in \mathrm{D}_{N}} Z_{\mu \nu}^{\lambda}(T)\left|S R_{(\pi / N) 00} T\right\rangle$.

Now $S$ is an element of the fundamental Voronoi cell $S \in \mathrm{F}_{\mathrm{SO}_{3} / \mathrm{D}_{2 N}}, \lambda$ labels an irreducible representation (irrep) of $\mathrm{D}_{N}$, and $Z_{\mu \nu}^{\lambda}(T)$ denotes the matrix elements of that representation, evaluated for the $\mathrm{D}_{N}$ element $T$. (The nonAbelian group $\mathrm{D}_{N}$ has both one-dimensional and twodimensional irreps.) The code words Eq. (80) correspond to $|\overline{0}\rangle=\mid \mathrm{D}_{N} ; 000$ and $|\overline{\mathbf{1}}\rangle=\left|R_{(\pi / N) 00} \mathrm{D}_{N} ;{ }_{00}^{\mathbf{1}}\right\rangle$, where $\lambda=\mathbf{1}$ is the trivial irrep. The basis states $\left|S \mathrm{D}_{N} \cdot{ }_{\mu \nu}\right\rangle$ span all states that can be reached when a correctable error acts on the code word $|\overline{0}\rangle ; S$ is the rotation error, and $\underset{\mu \nu}{\lambda}$ indexes the momentum kick. Similarly, the basis states $\left|S R_{(\pi / N) 00} \mathrm{D}_{N} ;{ }_{\mu \nu}^{\lambda}\right\rangle$ span all states that can be reached when a correctable error acts on the code word $|\overline{1}\rangle$. 


\section{Position shifts}

Our recovery consists of first correcting position shifts by measuring $S$ and applying $\vec{X}_{S}^{\dagger}$ to map all error states into the subspace $\left\{\left|\mathrm{D}_{N} ;{ }_{\mu \nu}\right\rangle,\left|R_{(\pi / N) 00} \mathrm{D}_{N} ;{ }_{\mu \nu}\right\rangle\right\}$ for all ${ }_{\mu \nu}^{\lambda}$. To extract $S$, we can readily adapt the procedure described in Sec. V B. This adaptation entails initializing an ancillary system in a $\mathrm{D}_{2 N}$-invariant state, performing the CROT gate (72), and measuring the ancilla in a basis of generalized spin-coherent states parameterized by $\mathrm{F}_{\mathrm{SO}_{3} / \mathrm{D}_{2 N}}$. A similar scheme can be used for state initialization.

\section{Momentum kicks}

To see how the momentum-kick operators $\hat{D}_{m n}^{\ell}$ affect the code words, we need to understand how the irrep of $\mathrm{SO}_{3}$ with angular momentum $\ell$ decomposes into irreps of $\mathrm{D}_{2 N}$ and $\mathrm{D}_{N}$. When $N$ is odd, the group $\mathrm{D}_{N}$ has two onedimensional irreps: the trivial representation (which we denote by $\mathbf{1}$ ) and a nontrivial representation (denoted by $\mathbf{1}^{\prime}$ ) which represents rotations by +1 and reflections by -1 . In addition, there are $(N-1) / 2$ two-dimensional irreps, which we denote by $\boldsymbol{2}_{k}$ with $k \in\{1,2, \ldots,(N-1) / 2\}$. We can characterize a representation according to how the generators of $\mathrm{D}_{N}$, the rotation $R_{(2 \pi / N) 00}$ and the reflection $R_{(2 \pi / N) \pi 0}$, are represented. All $\boldsymbol{2}_{k}$ irreps represent the reflection by the $2 \times 2$ matrix

$$
Z^{2_{k}}\left(R_{(2 \pi / N) \pi 0}\right)=\left(\begin{array}{ll}
0 & 1 \\
1 & 0
\end{array}\right)
$$

while the rotation is represented by the diagonal matrix

$$
Z^{\mathbf{2}_{k}\left(R_{(2 \pi / N) 00}\right)}=\left(\begin{array}{cc}
e^{i(2 \pi / N) k} & 0 \\
0 & e^{-i(2 \pi / N) k}
\end{array}\right) .
$$

Thus, the two-dimensional irrep of $\mathrm{D}_{N}$ decomposes as two one-dimensional irreps of $\mathrm{Z}_{N}$ which are interchanged by the reflection. When $N$ is even, there are $N / 2-1$ twodimensional irreps described by Eqs. (82) and (83) and also two additional one-dimensional irreps (denoted by $\mathbf{1}^{ \pm}$), representing the rotation by -1 and the reflection by \pm 1 , respectively.

The irrep $D^{\ell}$ of $\mathrm{SO}_{3}$ decomposes into $2 \ell+1$ onedimensional irreps of $\mathrm{U}_{1}$; these represent the rotation by angle $\phi$ about the $\mathbf{z}$ axis by $\left\{e^{i m \phi}, m=-\ell,-\ell+1, \ldots, \ell\right\}$. For $\ell<N / 2$ and $m \neq 0$, the $\pm m$ irreps of $\mathrm{U}_{1}$ pair up to form a two-dimensional irrep of $\mathrm{D}_{N}$, while the $m=0$ irrep of $\mathrm{U}_{1}$ provides a one-dimensional irrep, either $\mathbf{1}^{\prime}$ if $\ell$ is odd or $\mathbf{1}$ if $\ell$ is even. From Eq. (83), we can infer the "branching rules" specifying how the irreps of $\mathrm{D}_{2 N}$ transform under the $\mathrm{D}_{N}$ subgroup, namely, $\mathbf{1} \rightarrow \mathbf{1}, \mathbf{1}^{\prime} \rightarrow \mathbf{1}^{\prime}$, and

$$
\mathbf{2}_{k} \rightarrow \mathbf{2}_{k}, \quad \text { for } k<N / 2
$$

This result means that, of the irreps of $\mathrm{D}_{N}$ that arise in the decomposition of $D^{\ell}$ for $\ell<N / 2$, each is descended from a unique irrep of $\mathrm{D}_{2 N}$.

Suppose, now, that the momentum-kick operator $\hat{D}_{m n}^{\ell}$ acts on the code words Eq. (80) of the code associated with $\mathrm{D}_{N} \subset \mathrm{D}_{2 N}$, where $\ell<N / 2$, and we are able to diagnose the irrep of $\mathrm{D}_{N}$ according to which the damaged states transform. Because this irrep of $\mathrm{D}_{N}$ points to a unique irrep of $\mathrm{D}_{2 N}$, the action of the rotation $R_{(\pi / N) 00}$ on the states is unambiguously determined. This result means it is possible to recover, mapping $\hat{D}_{m n}^{\ell}|\overline{0}\rangle$ back to $|\overline{0}\rangle$ and $\hat{D}_{m n}^{\ell}|\overline{1}\rangle$ back to $|\overline{1}\rangle$, without any logical error.

However, for $\ell \geq N / 2$, the situation is different; the $\mathrm{D}_{2 N}$ irrep from which the $\mathrm{D}_{N}$ irrep arises is no longer unique. Correspondingly, projecting onto an irrep of $\mathrm{D}_{N}$ after $\hat{D}_{m n}^{\ell}$ acts does not fix the relative phase of $|\overline{0}\rangle$ and $|\overline{1}\rangle$; therefore, perfect recovery is not possible.

To be concrete, consider the case $\mathrm{D}_{3} \subset \mathrm{D}_{6} \subset \mathrm{SO}_{3}$. Here, $\mathrm{D}_{3}$ has just one two-dimensional irrep, which we call 2 . The $\ell=1$ irrep of $\mathrm{SO}_{3}$ decomposes as

$$
D^{\ell=1} \rightarrow \mathbf{1}^{\prime} \oplus \mathbf{2}_{1} \rightarrow \mathbf{1}^{\prime} \oplus \mathbf{2}
$$

under $\mathrm{D}_{6}$ and $\mathrm{D}_{3}$, respectively. The four matrix elements $D_{m n}^{1}$, for $m, n \in\{ \pm 1\}$, constitute the two-dimensional irrep 2 of $\mathrm{D}_{3}$ and $\mathbf{2}_{1}$ of $\mathrm{D}_{6}$; therefore, using the pairs of basis states (81), we have

$$
\begin{aligned}
\hat{D}_{m n}^{1}|\overline{0}\rangle & =\left|\mathrm{D}_{3} ; ;_{\mu=m, \nu=n}^{2}\right\rangle, \\
\hat{D}_{m n}^{1}|\overline{1}\rangle & =\exp \left(i \frac{\pi}{3} m\right)\left|R_{(\pi / 3) 00} \mathrm{D}_{3} ; \underset{\mu=m, \nu=n}{2}\right\rangle,
\end{aligned}
$$

for $m, n \in\{ \pm 1\}$. The damage inflicted on the code words by $\hat{D}_{m n}^{1}$ can be reversed by applying $\hat{D}_{m n}^{1 \dagger}$. (The error operator $\hat{D}_{00}^{1}$, realizing the representation $\mathbf{1}^{\prime}$ of $\mathrm{D}_{3}$ and $\mathrm{D}_{6}$, is also easily reversed.) Though $\hat{D}_{m n}^{1 \dagger}$ are not unitary operators acting on the full $\mathrm{SO}_{3}$ Hilbert space, a completely positive recovery map can be constructed which consists of projections onto $D_{3}$ irreps followed by appropriate momentum kicks. This map successfully recovers from any noise channel that can be expanded in $\left\{\hat{D}_{m n}^{\ell}\right\}$ for $\ell \leq 1$.

The $\mathrm{SO}_{3}$ irrep $\ell=2$, on the other hand, branches as

$$
D^{\ell=2} \rightarrow \mathbf{1} \oplus \mathbf{2}_{1} \oplus \mathbf{2}_{2} \rightarrow \mathbf{1} \oplus \mathbf{2} \oplus \mathbf{2}
$$

under $\mathrm{D}_{6}$ and $\mathrm{D}_{3}$ respectively. Now $D_{m n}^{2}$ constitutes the $\mathbf{2}_{2}$ irrep of $\mathrm{D}_{6}$ for $m, n \in\{ \pm 2\}$ and the $2_{1}$ irrep of $\mathrm{D}_{6}$ for $m, n \in\{ \pm 1\}$; however, these two distinct irreps of $\mathrm{D}_{6}$ cannot be distinguished as irreps of $\mathrm{D}_{3}$. Therefore, diagnosing the irrep of $\mathrm{D}_{3}$ according to which the damaged code words transform does not suffice to determine the relative phase of the two code basis states; now, 
TABLE II. Dihedral codes. Check operators for the $\mathrm{D}_{3} \subset \mathrm{D}_{6}$ codes and their corresponding eigenvalues and eigenstates within the 12-dimensional subspace $|R\rangle$ for $R \in \mathrm{D}_{6}$, where $a \in\left\{I, R_{(\pi / N) 00}\right\}$.

\begin{tabular}{lcc}
\hline \hline & $\bar{X}_{0, \pi, 0}$ & $\vec{X}_{(2 \pi / 3), 0,0}$ \\
\hline$\left|a \mathrm{D}_{3} ;{ }_{00}^{1}\right\rangle$ & +1 & +1 \\
$\left|a \mathrm{D}_{3} ; \mathbf{1}_{00}^{\prime}\right\rangle$ & -1 & +1 \\
$(1 / \sqrt{2})\left(\left|a \mathrm{D}_{3} ;_{+1,+1}^{2}\right\rangle \pm\left|a \mathrm{D}_{3} ;_{+1,-1}^{2}\right\rangle\right)$ & \pm 1 & $e^{-i(2 \pi / 3)}$ \\
$(1 / \sqrt{2})\left(\left|a \mathrm{D}_{3} ; ;_{-1,+1}^{2}\right\rangle \pm\left|a \mathrm{D}_{3} ; ;_{-1,-1}^{2}\right\rangle\right)$ & \pm 1 & $e^{i(2 \pi / 3)}$ \\
\hline \hline
\end{tabular}

$$
\begin{gathered}
\hat{D}_{m n}^{2}|\overline{0}\rangle=\left|\mathrm{D}_{3} ; ;_{\mu=m, \nu=n}^{2}\right\rangle, \\
\hat{D}_{m n}^{2}|\overline{1}\rangle=\exp \left(i \frac{2 \pi}{3} m\right)\left|R_{(\pi / 3) 00} \mathrm{D}_{3} ;{ }_{\mu=m, \nu=n}^{2}\right\rangle,
\end{gathered}
$$

for $m, n \in\{ \pm 2\}$, in contrast to Eq. (86). If, say, a $\hat{D}_{22}^{2}$ error were to occur, it could be mistaken for a $\hat{D}_{-1,-1}^{1}$ error. An attempt to recover by applying $\hat{D}_{-1,-1}^{1 \dagger}$ results in a logical phase error, with $|\overline{0}\rangle \rightarrow|\overline{0}\rangle$ and $|\overline{1}\rangle \rightarrow-|\overline{1}\rangle$. Thus, while the $\mathrm{D}_{3} \subset \mathrm{D}_{6}$ code can protect against angular-momentum kicks with $\ell \leq 1$, it does not protect against arbitrary kicks with $\ell \leq 2$. In general, the observation (84) implies that the $\mathrm{D}_{N} \subset \mathrm{D}_{2 N}$ code protects against all kicks with $\ell<N / 2$.

An undetectable error corresponds to a nontrivial representation of $\mathrm{D}_{6}$ that branches to the trivial representation of $\mathrm{D}_{3}$. The lowest angular momentum at which this branching occurs is $\ell=3$, with branching rules

$$
\begin{aligned}
D^{\ell=3} & \rightarrow \mathbf{1}^{+} \oplus \mathbf{1}^{-} \oplus \mathbf{1}^{\prime} \oplus \mathbf{2}_{1} \oplus \mathbf{2}_{2} \\
& \rightarrow \mathbf{1} \oplus \mathbf{1}^{\prime} \oplus \mathbf{1}^{\prime} \oplus \mathbf{2} \oplus \mathbf{2} .
\end{aligned}
$$

Here, the nontrivial irrep $\mathbf{1}^{+}$of $\mathrm{D}_{6}$ reduces to the trivial irrep 1 of $\mathrm{D}_{3}$. Diagnosing this trivial irrep yields no information about its parent irrep of $\mathrm{D}_{6}$, meaning that $\ell=3$ momentum kicks produce undetectable errors.

For $N=3$, the only nontrivial correction one needs while mapping the states back into the code space is the correction of the $\mu$-dependent phase (86b) for $\lambda=\mathbf{2}$. Therefore, only knowledge of $\lambda$ and $\mu$ is required, and the error syndrome can be obtained by performing a projective measurement onto a basis that resolves these indices without extracting the logical information. One such basis is the joint eigenbasis of the two commuting rotations from Table II. Such a measurement projects the corrupted code words onto eigenstates of these operators. A successful recovery operation, then, maps the resulting states back into the code space, applying (in the case of $\lambda=2)$ a $\mu$-dependent phase that undoes the action of the $\ell=1$ momentum kick from Eq. (86b).

\section{E. Other non-Abelian molecular codes}

Other interesting codes can be constructed using the tetrahedral, octahedral, and icosahedral subgroups of the rotation group. All are finite non-Abelian groups, denoted $\mathrm{T}, \mathrm{O}$, and $\mathrm{I}$, respectively, with order $|\mathrm{T}|=12,|\mathrm{O}|=24$, and $|\mathrm{I}|=60$. T is isomorphic to the alternating group $\mathrm{A}_{4}, \mathrm{O}$ is isomorphic to the permutation group $\mathrm{S}_{4}$, and $\mathrm{I}$ is isomorphic to $A_{5}$. Since $T$ is a subgroup of both $\mathrm{O}$ and $\mathrm{I}$, codes can be constructed based on the embedding $\mathrm{T} \subset \mathrm{O}$, with code dimension 2, or based on $\mathrm{T} \subset \mathrm{I}$, with code dimension 5 .

The logical code words for $\mathrm{T} \subset \mathrm{O}$ are uniform superpositions of $\mathrm{SO}_{3}$ elements, indicated as black and white balls in Fig. 4(b). That code can correct rotation errors in the fundamental Voronoi cell $\mathrm{F}_{\mathrm{SO}_{3} / \mathrm{O}}$, the cubelike region bounded in blue in the figure. The $\mathrm{T} \subset \mathrm{I}$ code can correct rotation errors in $\mathrm{F}_{\mathrm{SO}_{3} / \mathrm{I}}$, the dodecahedron bounded in blue in Fig. 4(c). In that figure, the balls of five different colors correspond to the $\mathrm{SO}_{3}$ elements making up this code's five logical code words.

To investigate how well these codes protect against momentum kicks, we examine the branching rules for $\mathrm{SO}_{3} \rightarrow \mathrm{K} \rightarrow \mathrm{H}$, as in Sec. V D, but where now $\mathrm{H}=\mathrm{T}$ and $\mathrm{K}$ is either $\mathrm{O}$ or $\mathrm{I}$. The group $\mathrm{T}$ has four irreps labeled as $\left\{\mathbf{1}, \mathbf{1}^{\prime}, \mathbf{1}^{\prime \prime}, \mathbf{3}\right\}$ (with the number denoting dimension), $\mathrm{O}$ has five irreps $\left\{\mathbf{1}, \mathbf{1}^{\prime}, \mathbf{2}, \mathbf{3}, \mathbf{3}^{\prime}\right\}$, and I has five irreps $\left\{\mathbf{1}, \mathbf{3}, \mathbf{3}^{\prime}, \mathbf{4}, \mathbf{5}\right\}$. We note that $D^{\ell=1}$, the defining threedimensional irrep of $\mathrm{SO}_{3}$, also provides defining irreps of the subgroups $\mathrm{T}, \mathrm{O}$, and $\mathrm{I}$; therefore, the branching rule

$$
D^{\ell=1} \rightarrow \mathbf{3} \rightarrow \mathbf{3}
$$

applies to both the $\mathrm{T} \subset \mathrm{O}$ and $\mathrm{T} \subset \mathrm{I}$ codes. This result means that projecting onto the basis of irreps of $\mathrm{T}$ unambiguously identifies the error $\hat{D}_{m n}^{\ell \leq 1}$, which can, therefore, be corrected. Hence, both codes protect against kicks with $\ell \leq 1$.

Focusing on $\mathrm{T} \subset \mathrm{O}$ (the $\mathrm{T} \subset \mathrm{I}$ code behaves similarly), the $\ell=2$ irrep of $\mathrm{SO}_{3}$ has branching rules [159]

$$
D^{\ell=2} \rightarrow \mathbf{2} \oplus \mathbf{3}^{\prime} \rightarrow \mathbf{1}^{\prime} \oplus \mathbf{1}^{\prime \prime} \oplus \mathbf{3} .
$$

Here, the T-irrep 3 is the same 3D irrep that appears in Eq. (90), but the irrep $\mathbf{3}^{\prime}$ of $\mathrm{O}$ is different than the irrep $\mathbf{3}$ in Eq. (90). Therefore, the projection onto the basis of irreps of $\mathrm{T}$ does not unambiguously identify the irrep of $\mathrm{O}$, and we conclude that the $\mathrm{T} \subset \mathrm{O}$ codes do not protect against arbitrary momentum kicks with $\ell \leq 2$.

Undetectable errors are associated with nontrivial irreps of $\mathrm{O}$ which branch to trivial irreps of $\mathrm{T}$. This branching occurs at $\ell=3$, due to the branching rules

$$
D^{\ell=3} \rightarrow \mathbf{1}^{\prime} \oplus \mathbf{3} \oplus \mathbf{3}^{\prime} \rightarrow \mathbf{1} \oplus \mathbf{3} \oplus \mathbf{3} .
$$

Interestingly, the $\mathrm{T} \subset \mathrm{O}$ code can also detect all momentum kicks with $\ell=4,5$, because, for these irreps of $\mathrm{SO}_{3}$, the 
trivial irrep of T appears only as a descendant of the trivial irrep of $\mathrm{O}$.

Momentum-kick correction for the more general codes proceeds by measuring a combination of left and right rotations that distinguishes the error spaces sufficiently well for one to correct successfully. As with the dihedral codes in Eq. (86), momentum kicks produce $\mu$-dependent relative phases between corrupted code words, which need to be corrected upon recovery. Using $\mathrm{T} \subset \mathrm{O}$ as an example, successful recovery requires determining the irrep label $\lambda \in$ $\left\{\mathbf{1}, \mathbf{1}^{\prime}, \mathbf{1}^{\prime \prime}, \mathbf{3}\right\}$ and the $\mu$ label for $\lambda=\mathbf{3}$. After correcting position shifts, the corrupted states lie in the subspace $\{|R\rangle, R \in \mathrm{O}\}$. The three check operators $\overleftarrow{X}_{(\pi / 2)(\pi / 2) \pi}, \vec{X}_{0 \pi 0}$, and $\vec{X}_{\pi 00}$ commute on this space, and measuring in their joint eigenbasis resolves $\lambda$ and $\mu$.

Just like $\mathrm{SO}_{3}$ rotations permute cosets in the lens space $\mathrm{SO}_{3} / Z_{N}$ in an induced representation (50), elements $\left\{\vec{X}_{S}\right\}_{S \in \mathrm{K}}$ permute cosets in $\mathrm{K} / \mathrm{H}$, providing logical $X$-type operators. For T $\subset \mathrm{O}$, there are only two cosets, so $\left\{\vec{X}_{S}\right\}_{S \in \mathrm{O}}$ either act trivially or exchange the two code words. For $\mathrm{T} \subset \mathrm{I}$, the 60 rotations $\left\{\vec{X}_{S}\right\}_{S \in \mathrm{I}}$ form a five-dimensional permutation representation of I when acting on the five code words. Since $I=A_{5}$ (the alternating group), any permutations of the code words in $\mathrm{A}_{5}$ are realized by the unitary $\vec{X}_{R}$ 's. Moreover, these gates are fault tolerant. If there is a slight over- or underrotation $S^{\prime} \neq S$ and the rotated state $\vec{X}_{S^{\prime}}|\bar{r}\rangle$ is close to (but not quite equal to) the code word $\vec{X}_{S}|\vec{r}\rangle$, then the error-correction procedure fixes this fault by mapping $\vec{X}_{S^{\prime}}|\bar{r}\rangle$ to the closest code word.

\section{LINEAR-ROTOR CODES}

By a linear rotor, we mean a rigid body with a symmetry axis, such that rotations about that axis leave the orientation of the body invariant. The paradigmatic example is a diatomic molecule containing two distinct atoms; we discuss other manifestations in Sec. III C. In contrast to an asymmetric body, for which orientations of the body are in one-to-one correspondence with elements of the rotation group $\mathrm{SO}_{3}$, the configuration space of the linear rotor is the coset space $\mathrm{SO}_{3} / \mathrm{U}_{1}=\mathrm{S}^{2}$, because the $\mathrm{U}_{1}$ rotations about the symmetry axis do not alter the orientation. This example is equivalent to the configuration space of a particle moving on a two-sphere.

The position eigenstates $\{|\mathbf{v}\rangle\}$ provide an orthogonal basis for the Hilbert space of the linear rotor, with continuum normalization, where $\mathbf{v}$ denotes a point on $S^{2}$ (equivalently, a unit 3-vector). It is convenient to parametrize points on the sphere using spherical coordinates $\mathbf{v}=(\theta, \phi)$, where $\theta$ denotes the polar angle and $\phi$ is the azimuthal angle; thus, $\theta \in[0, \pi]$ and $\phi \in[0,2 \pi)$.

A rotation $R \in \mathrm{SO}_{3}$ rotates the linear rotor with orientation $\mathbf{v}$ to a new orientation $R \mathbf{v}$. It is represented by the unitary operator $\hat{X}_{R}$, with action

$$
\hat{X}_{R}|\mathbf{v}\rangle=|R \mathbf{v}\rangle .
$$

A rotation acting on $\mathrm{S}^{2}$, in contrast to a rotation acting on states of an asymmetric rigid rotor, has fixed points; the position eigenstate $|\mathbf{v}\rangle$ is left invariant by a rotation $R=$ $(\omega, \pm \mathbf{v})$ about the axis $\mathbf{v}$ or the axis $-\mathbf{v}$ :

$$
\hat{X}_{\omega, \pm \mathbf{v}}|\mathbf{v}\rangle=|\mathbf{v}\rangle .
$$

Another relevant operation is inversion or parity, mapping $\mathbf{v}$ to its antipode $-\mathbf{v}$. Rotations together with inversions generate the group $\mathrm{O}_{3}$ of proper and improper rotations in three dimensions, isomorphic to $\mathrm{SO}_{3} \times \mathrm{Z}_{2}$. In Hilbert space, $P$ is represented by $\hat{X}_{P}$, with action

$$
\hat{X}_{P}|\mathbf{v}\rangle=|-\mathbf{v}\rangle,
$$

which commutes with $\hat{X}_{R}$ for any $R$.

Dual to the continuous position basis is the discrete Fourier-conjugate basis, defined on $S^{2}$ by

$$
\begin{aligned}
& \left.|\mathbf{v}\rangle=\left.\sum_{\ell \geq 0} \sum_{|m| \leq \ell} Y_{m}^{\ell_{\star}^{\star}}(\mathbf{v})\right|_{m} ^{\ell}\right\rangle, \\
& \left.\left|\begin{array}{l}
\ell \\
\mid
\end{array}=\int_{\mathrm{S}^{2}} d \mathbf{v} Y_{m}^{\ell}(\mathbf{v})\right| \mathbf{v}\right\rangle,
\end{aligned}
$$

where $Y_{m}^{\ell}(\mathbf{v})$ is a spherical harmonic. The momentum states satisfy the normalization

$$
\left\langle{ }_{m}^{\ell}||_{m^{\prime}}^{\ell^{\prime}}\right\rangle=\int_{\mathrm{S}^{2}} d \mathbf{v} Y_{m}^{\ell \star}(\mathbf{v}) Y_{m^{\prime}}^{\ell^{\prime}}(\mathbf{v})=\delta_{\ell \ell^{\prime}} \delta_{m m^{\prime}},
$$

where $d \mathbf{v}$ is the surface area element on the two-sphere. In the momentum basis,

$$
\begin{aligned}
& \left.\hat{X}_{R}=\left.\sum_{\ell \geq 0} \sum_{|m| \leq \ell} D_{m n}^{\ell \star}(R)\right|_{m} ^{\ell}\right\rangle\left\langle\left\langle{ }_{n}^{\ell}\right|,\right. \\
& \hat{X}_{P}=\sum_{\ell \geq 0} \sum_{|m| \leq \ell}(-1)^{\ell}\left|{ }_{m}^{\ell}\right\rangle\left\langle{ }_{m}^{\ell}\right| .
\end{aligned}
$$

Other relevant features of $\mathrm{S}^{2}$ are listed in the third column in Table V.

The spherical harmonics form a basis for functions on the sphere, meaning that any operator on $S^{2}$ that is diagonal in the position basis can be expanded in $Z$-type operators

$$
\hat{Y}_{m}^{\ell}=\int_{\mathrm{S}^{2}} d \mathbf{v}|\mathbf{v}\rangle Y_{m}^{\ell}(\mathbf{v})\langle\mathbf{v}| .
$$

However, since there are more rotations than molecular orientations, products of rotations and the above diagonal $Z$-type operators do not form an orthonormal basis for operators on $S^{2}$. They instead form an overcomplete frame, satisfying the completeness relation in Eqs. (123): 
$\frac{1}{2 \pi} \int_{\mathrm{SO}_{3}} d R \sum_{\ell, m}\left\langle\mathbf{v}\left|\hat{X}_{R} \hat{Y}_{m}^{\ell}\right| \mathbf{w}\right\rangle\left\langle\mathbf{w}^{\prime}\left|\hat{Y}_{m}^{\ell \dagger} \hat{X}_{R}^{\dagger}\right| \mathbf{v}^{\prime}\right\rangle=\delta_{\mathbf{v v}^{2}}^{\mathrm{S}^{2}}{ }^{\delta_{\mathbf{w w}}^{\prime}} \mathrm{S}^{2}$

A similar relation holds for more general quotient spaces, described in Appendix D. Overcompleteness complicates the analysis of recovery from errors of the form

$$
\rho \rightarrow \hat{X}_{R} \hat{Y}_{m}^{\ell} \rho \hat{Y}_{m}^{\ell \dagger} \hat{X}_{R}^{\dagger}
$$

in which a momentum kick by $\ell, m$ is combined with a rotation $R$.

\section{A. Simplest linear-rotor codes}

Here, we embed the $\mathrm{Z}_{N} \subset \mathrm{Z}_{2 N}$ code (20) for general $N$ into the linear rotor. While their $\mathrm{SO}_{3}$ counterparts allow protection against small momentum and position shifts, these codes can correct either against rotations around any axis by sufficiently small angles or against $O(N / 2)$ angular-momentum kicks.

\section{Code words}

Constructing the simplest linear-rotor codes is similar to that for $\mathrm{SO}_{3}$ in Sec. VA. Code words are equal superpositions of equatorial states $|(\pi / 2), \phi\rangle$, whose azimuthal angle $\phi$ is every even or odd multiple of $\pi / N$ :

$$
\begin{aligned}
& |\overline{0}\rangle=\frac{1}{\sqrt{N}} \sum_{h \in Z_{N}}\left|\frac{\pi}{2}, \frac{2 \pi}{N} h\right\rangle, \\
& |\overline{1}\rangle=\frac{1}{\sqrt{N}} \sum_{h \in Z_{N}}\left|\frac{\pi}{2}, \frac{2 \pi}{N} h+\frac{\pi}{N}\right\rangle .
\end{aligned}
$$

For the case $N=3$, these code words are shown in Fig. 2(c). These code words are not normalizable, but normalizable approximate versions can be obtained by introducing a damping factor, just as we discuss for $\mathrm{SO}_{3}$ codes (see Sec. V C and Appendix A).

Expressing the code words in terms of angularmomentum states $\left.\left.\right|_{m} ^{\ell}\right\rangle$ (96b) yields, for $r \in\{0,1\}$,

$$
\left.|\bar{r}\rangle=\sqrt{N} \sum_{\ell \geq 0} \sum_{|p N| \leq \ell}(-1)^{p r} Y_{p N}^{\ell}\left(\frac{\pi}{2}, 0\right)||_{p N}^{\ell}\right\rangle .
$$

To derive Eq. (103), it suffices to observe that

$$
Y_{m}^{\ell}\left(\frac{\pi}{2}, \phi\right)=Y_{m}^{\ell}\left(\frac{\pi}{2}, 0\right) e^{i m \phi} .
$$

Therefore, the only terms that survive when we do the sum over $h \in \mathrm{Z}_{N}$ in Eq. (103) are those in which $m$ is an integer multiple of $N$.
The logical- $X$ code words are

$$
\begin{aligned}
|\overline{0}\rangle_{X} & \left.=\sqrt{2 N} \sum_{\ell \geq 0} \sum_{|2 p N| \leq \ell} Y_{2 p N}^{\ell}\left(\frac{\pi}{2}, 0\right)||_{2 p N}^{\ell}\right\rangle, \\
|\overline{1}\rangle_{X} & \left.=\left.\sqrt{2 N} \sum_{\ell \geq 0} \sum_{|(2 p+1) N| \leq \ell} Y_{(2 p+1) N}^{\ell}\left(\frac{\pi}{2}, 0\right)\right|_{(2 p+1) N} ^{\ell}\right\rangle ;
\end{aligned}
$$

that is, $|\overline{0}\rangle_{X}$ is a superposition of angular-momentum eigenstates with $m$ an even multiple of $N$, and $|\overline{1}\rangle_{X}$ is a superposition of states with $m$ an odd multiple of $N$. In addition, because $Y_{m}^{\ell}[(\pi / 2), \phi]=0$ whenever $\ell-m$ is odd [109], only every other value of $\ell$ appears in the superposition for each fixed value of $m$ [see Fig. 2(c)].

\section{Position shifts}

We use the error-correction conditions [160,161] (see also Ref. [162], Theorem 10.1) to determine which errors can be handled by our code. To be able to correct against some subset of rotations, one should satisfy for all such correctable rotations $R, R^{\prime}$ :

$$
\begin{aligned}
\left\langle\overline{0}\left|\hat{X}_{R}^{\dagger} \hat{X}_{R^{\prime}}\right| \overline{0}\right\rangle & =\left\langle\overline{1}\left|\hat{X}_{R}^{\dagger} \hat{X}_{R^{\prime}}\right| \overline{1}\right\rangle, \\
\left\langle\overline{0}\left|\hat{X}_{R}^{\dagger} \hat{X}_{R^{\prime}}\right| \overline{1}\right\rangle & =0 .
\end{aligned}
$$

This product of rotations is just another $\mathrm{SO}_{3}$ rotation, $\hat{X}_{R}^{\dagger} \hat{X}_{R^{\prime}}$, rotating the equatorial "necklace" of constituent orientations of our code words to another great circle.

To satisfy Eq. (106a), notice that, if $N$ is odd, the code word $|\overline{1}\rangle$ consists of superpositions of all points antipodal to those of the code word $|\overline{0}\rangle$ :

$$
|\overline{1}\rangle=\hat{X}_{P}|\overline{0}\rangle \text {. }
$$

Therefore, assuming odd $N$ from now on and remembering that inversion commutes with all rotations,

$$
\left\langle\overline{1}\left|\hat{X}_{R}^{\dagger} \hat{X}_{R^{\prime}}\right| \overline{1}\right\rangle=\left\langle\overline{0}\left|\hat{X}_{P} \hat{X}_{R}^{\dagger} \hat{X}_{R^{\prime}} \hat{X}_{P}\right| \overline{0}\right\rangle=\left\langle\overline{0}\left|\hat{X}_{R}^{\dagger} \hat{X}_{R^{\prime}}\right| \overline{0}\right\rangle .
$$

With the antipodal assumption (107), the first condition (106a) is satisfied for all $R \in \mathrm{SO}_{3}$.

The second condition (106b) puts restrictions on where the rotations can map the code words. To be concrete, consider the case $N=3$, depicted in Fig. 2(c). The code word $|\overline{0}\rangle$ is a uniform superposition of three "constituent" points on the equator of $\mathrm{S}^{2}$, which are marked by black balls in the figure. The code word $|\overline{1}\rangle$ is likewise a uniform superposition of three constituent points, marked by white 
balls. For each constituent point, there is a corresponding Voronoi cell, containing all points on $\mathrm{S}^{2}$ which are closer to that point than to any other constituent point (see Appendix B). Each such Voronoi cell is a spherical lune, a sliver of the sphere bounded by two lines of longitude separated by angle $\pi / 3$; one of these cells is colored blue in Fig. 2(c). The condition Eq. (106b) surely is satisfied as long as the rotation $R^{-1} R^{\prime}$ maps each constituent point to a point in its Voronoi cell.

\section{Momentum kicks}

Just like rigid-rotor codes, linear-rotor codes protect against sufficiently small momentum kicks. Selection rules for addition of angular momenta dictate that a momentumkick operator $\hat{Y}_{m}^{\ell}$ maps a momentum state $\left.\left.\right|_{m^{\prime}} ^{\ell^{\prime}}\right\rangle$ to states $\left.\left.\right|_{M} ^{L}\right\rangle$ that satisfy

$$
\left|\ell-\ell^{\prime}\right| \leq L \leq\left|\ell+\ell^{\prime}\right|, \quad M=m+m^{\prime}
$$

Because the code words have support on states such that $m$ is an integer multiple of $N$, the code can detect momentum kicks with $\ell \leq N-1$ and correct shifts with $\ell<N / 2$. The procedure for diagnosing and correcting momentum kicks follows closely the corresponding discussion for rigidrotor codes.

\section{Combined shifts}

We have now seen that the code with basis states Eq. (102) can protect against both small rotations and small angular-momentum kicks. But problems arise when we consider errors that combine a rotation and a kick. Suppose, for example, that $\mathbf{v}$ is a constituent point of the code word $|\overline{0}\rangle$, and, hence, $-\mathbf{v}$ is a constituent point of $|\overline{1}\rangle$, and consider a rotation $R_{\omega, \mathbf{v}}$ about the axis $\mathbf{v}$ by a small nonzero angle $\omega$. Then, because one and only one constituent point of each code word is preserved by the rotation, we have

$$
\begin{aligned}
\left\langle\overline{0}\left|\hat{Y}_{m}^{\ell} \hat{X}_{R}\right| \overline{0}\right\rangle & =\frac{1}{N} Y_{m}^{\ell}(\mathbf{v}), \\
\left\langle\overline{1}\left|\hat{Y}_{m}^{\ell} \hat{X}_{R}\right| \overline{1}\right\rangle & =\frac{1}{N} Y_{m}^{\ell}(-\mathbf{v})=\frac{1}{N}(-1)^{\ell} Y_{m}^{\ell}(\mathbf{v}) .
\end{aligned}
$$

To be specific, $Y_{1}^{1}(\theta, \phi) \propto e^{i \phi} \sin \theta$ is nonzero for $\theta=\pi / 2$, and we therefore conclude that $\left\langle\overline{0}\left|\hat{Y}_{1}^{1} \hat{X}_{R}\right| \overline{0}\right\rangle \neq\left\langle\overline{1}\left|\hat{Y}_{1}^{1} \hat{X}_{R}\right| \overline{1}\right\rangle$. This conclusion means that the error-correction condition is not satisfied by this code for this error.

More generally, suppose that $\mathbf{v}_{1}$ is a constituent point of $|\overline{0}\rangle$ and that $R, R^{\prime}$ are two rotations, both of which map $\mathbf{v}_{1}$ to another point $\mathbf{v}_{2}$. (There is a one-parameter family of such rotations.) Suppose, in addition, that $R \mathbf{u} \neq R \mathbf{u}^{\prime}$, where $\mathbf{u}$ and $\mathbf{u}^{\prime}$ are any other constituent points of $|\overline{0}\rangle$. Then,

$$
\begin{aligned}
\left\langle\overline{0}\left|\hat{X}_{R}^{\dagger} \hat{Y}_{m}^{\ell} \hat{X}_{R^{\prime}}\right| \overline{0}\right\rangle & =\frac{1}{N} Y_{m}^{\ell}\left(\mathbf{v}_{2}\right), \\
\left\langle\overline{1}\left|\hat{X}_{R} \hat{Y}_{m}^{\ell} \hat{X}_{R}\right| \overline{1}\right\rangle & =\frac{1}{N} Y_{m}^{\ell}\left(-\mathbf{v}_{2}\right)=\frac{1}{N}(-1)^{\ell} Y_{m}^{\ell}\left(\mathbf{v}_{2}\right) .
\end{aligned}
$$

Again, because for odd $\ell$ and nonzero $Y_{m}^{\ell}\left(\mathbf{v}_{2}\right)$ we find that $\left\langle\overline{0}\left|\hat{X}_{R}^{\dagger} \hat{Y}_{m}^{\ell} \hat{X}_{R^{\prime}}\right| \overline{0}\right\rangle \neq\left\langle\overline{1}\left|\hat{X}_{R}^{\dagger} \hat{Y}_{m}^{\ell} \hat{X}_{R^{\prime}}\right| \overline{1}\right\rangle$, the error-correction conditions are not satisfied.

Given the above limitations, this code can protect against either (I) all rotations $R \in \mathrm{SO}_{3}$ that keep each constituent orientation in its corresponding Voronoi cell, or (II) all momentum kicks $\hat{Y}_{m}^{\ell}$ with $0 \leq m \leq \ell<N / 2$. In addition, if we exclude from set (I) all rotations around axes corresponding to constituent points of our logical states, then the code can correct both the rotations remaining in (I) and momentum kicks (II). However, the code still cannot correct products of such rotations and kicks due to Eq. (111). This result is in contrast to rigid-rotor codes, which protect against any product of a sufficiently small rotation and momentum kick.

The above diminished performance begs the question of whether such codes are of any use against realistic noise [146-152]. Since the rotations themselves are overcomplete, and since these codes protect against (virtually) all small rotations, such codes may be applicable to certain environments, especially ones where the noise is biased [163]. These codes can also be concatenated with other codes, whose purpose is to provide a layer of protection against momentum kicks. It is likely that the formalism of approximate error correction $[3,164,165]$ may be required to study their applicability.

In a sense, the continuous $\mathrm{U}_{1}$ symmetry of the linear rotor is too much symmetry for such GKP-type codes to perform well. However, the framework presented here can serve as a springboard to designing codes for the many molecules with discrete symmetries-less symmetric than the linear rotor but not completely asymmetric like the rigid rotor (see Sec. III C). Configuration spaces of less symmetric molecules should make it possible for codes of this type to perform better (see Sec. VIII).

\section{Partial Fourier transform}

To construct a recovery for the above error sets, we can once again develop a partially Fourier-transformed basis. As before, we use subgroups $\mathrm{H} \subset \mathrm{K}$ to split up our underlying space $X=S^{2}$ into various pieces as

$$
\mathrm{S}^{2}=\bigcup_{\mathbf{w} \in \mathrm{S}^{2} / \mathrm{K}} \mathrm{Kw}=\bigcup_{\mathbf{w} \in \mathrm{S}^{2} / \mathrm{K}} \bigcup_{r \in \mathrm{K} / \mathrm{H}} r \mathrm{Hw}
$$

But, because $S^{2}$ is not a group, the first quotient space does not consist of cosets but instead consists of orbits in $S^{2}$ under $\mathrm{K}$. The orbit $\mathrm{Kw}$ of a point $\mathbf{w} \equiv(\theta, \phi)$ under $\mathrm{K}$ is the set of points to which one obtains by applying rotations in 
$\mathrm{K}$ to $(\theta, \phi)$. Identifying points in $\mathrm{S}^{2}$ belonging to the same orbit, one comes up with the orbit manifold (i.e., orbifold) $\mathrm{S}^{2} / \mathrm{K}$ (see Table I). We construct the partially Fouriertransformed basis on $\mathrm{S}^{2}$ and its corresponding recovery for the codes discussed above in Appendix E.

\section{Gates and check operators}

Check operators for the $Z_{N}$ code on $S^{2}$ are similar to their counterpart on $\mathrm{SO}_{3}$ : The $Z$-type check operator $\hat{S}_{Z}$ selects the $2 N$ orientations $\{|(\pi / 2),(\pi / N) h+\mu \pi\rangle\}_{h \in Z_{N}, \mu \in Z_{2}}$, while the $X$-type check operator $\hat{S}_{X}$ has a maximal eigenvalue only at the two particular superpositions of these orientations [corresponding to the two code words (102)].

The momentum shift $\hat{Y}_{2 N}^{2 N}(99)$ acts as the identity on the code space, while the shift $\hat{Y}_{N}^{N}$ acts as a logical- $Z$ operator. As with the rigid rotor, neither of these are unitary on the full Hilbert space. We can obtain simpler versions by using operators of the form [166-168]

$$
(\hat{\mathbf{v}} \cdot \mathbf{w})^{p} \equiv \int_{\mathrm{S}^{2}} d \mathbf{v}(\mathbf{v} \cdot \mathbf{w})^{p}|\mathbf{v}\rangle\langle\mathbf{v}|,
$$

where $\mathbf{w} \in \mathrm{S}^{2}$ and $p$ is a non-negative integer. Expressing the "position operator" $\hat{\mathbf{v}}$ in spherical coordinates yields the $\hat{S}_{Z}$ below:

$$
\begin{aligned}
& \hat{S}_{Z}=\cos (2 N \hat{\phi}) \sin ^{2 N} \hat{\theta}, \\
& \hat{S}_{X}=\cos \left(\frac{2 \pi}{N} \hat{L}_{z}\right) .
\end{aligned}
$$

$X$-type check operators include powers of the $\mathbf{z}$-axis rotation $\hat{X}_{(2 \pi / N), \mathbf{z}}=e^{-i(2 \pi / N) \mathbf{z} \cdot \hat{\boldsymbol{L}}}$, where $h \in \mathrm{Z}_{N}, \quad \hat{\boldsymbol{L}}=$ $\left(\hat{L}_{x}, \hat{L}_{y}, \hat{L}_{z}\right)$ is the angular-momentum operator, and $\left.\hat{L}_{z}\left|{ }_{m}^{\ell}\right\rangle=\left.m\right|_{m} ^{\ell}\right\rangle$. A combination of such powers yields Eq. (114b) above. Inversion $\hat{X}_{P}$ is a logical- $X$ operator.

\section{B. Non-Abelian subgroup codes}

Mimicking Sec. VE, we briefly discuss more general codes based on non-Abelian $\mathrm{H} \subset \mathrm{K}$. A simple example is $\mathrm{H}=\mathrm{T}$ and $\mathrm{K}=\mathrm{T} \times \mathrm{Z}_{2}^{P}$, where $\mathrm{Z}_{2}^{P}$ is the group generated by inversion $P$. Its code word constituents lie on two antipodal tetrahedra that are invariant under $\mathrm{T}$ (black and white points in Fig. 5, respectively). Taken together, these tetrahedra make up a cube. Letting $\mathbf{w}_{\text {cube }}$ be one of the vertices of the cube, we can express the code words in terms of the orbit of $\mathbf{w}_{\text {cube }}$ under $\mathrm{T}$ :

$$
\begin{gathered}
|\overline{0}\rangle=\frac{1}{6} \sum_{R \in \mathrm{T}}\left|R \mathbf{w}_{\text {cube }}\right\rangle, \\
|\overline{1}\rangle=\frac{1}{6} \sum_{R \in \mathrm{T}}\left|-R \mathbf{w}_{\text {cube }}\right\rangle .
\end{gathered}
$$
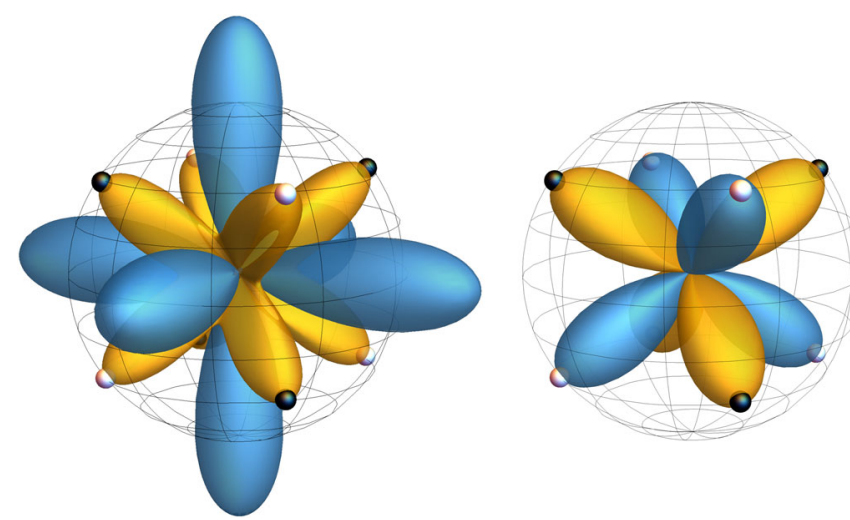

FIG. 5. Linear-rotor codes. Sketch of two polyhedral harmonics for the $\mathrm{T}$ code on $\mathrm{S}^{2}$, whose two code words are equal superpositions of the white and black points, respectively. The left harmonic is the code's check operator $\hat{S}_{Z}(116)$, and the right is the logical- $Z$ operator (117). Positive (negative) values are in yellow (blue), and the outlined spheres have radius 1 .

The normalization factor $1 / 6$ arises here because the 12 elements of $\mathrm{T}$ map $\mathbf{w}_{\text {cube }}$ to only four distinct constituents for each code word. As before, these code words are part of a partially Fourier-transformed basis associated with $\mathrm{T}$, formulated in Appendix E. These T codes correct against momentum shifts $\left\{\hat{D}_{m n}^{\ell}\right\}$ with $\ell \leq 1$ and detect momentum shifts with $\ell \leq 2$, like their counterparts on $\mathrm{SO}_{3}$ (see Sec. VE).

\section{Check operators}

The $Z$-type check operators $\hat{S}_{Z}$ have the same eigenvalue at each of the constituent points of the code, which in the T case means the corners of the cube in Fig. 5. This condition is clearly satisfied by harmonics that are symmetric under K, since that group leaves the cube invariant. In Appendix E, we describe how to obtain such harmonics by "averaging" or "twirling" the spherical harmonics over K. Using this procedure, we obtain the $Z$-type check operator

$\hat{S}_{Z}=\frac{3}{16}\left(30 \cos ^{2} \hat{\theta}-35 \cos ^{4} \hat{\theta}-5 \sin ^{4} \hat{\theta} \cos 4 \hat{\phi}-3\right)$,

corresponding to the K-symmetric harmonic $Y_{0}^{4}$. Shown in the left in Fig. 5, the above is normalized such that the constituent states $\left\{\left| \pm R \mathbf{w}_{\text {cube }}\right\rangle\right\}_{R \in \mathrm{K}}$ of the code words are eigenstates with eigenvalue +1 .

Naturally, T-symmetric harmonics can act as logical $Z$-type operators within the code space. The smallest $-\ell$ logical- $Z$ operator is shown in the right in Fig. 5, corresponding to the harmonic $Y_{2}^{3}$ averaged over $\mathrm{T}$,

$$
\bar{Z}=\frac{3 \sqrt{3}}{2} \sin ^{2} \hat{\theta} \cos \hat{\theta} \sin 2 \hat{\phi}
$$


The $X$-type check operators $\hat{S}_{X}$ consist of rotations $\left\{\hat{X}_{\omega, \mathbf{w}},(\omega, \mathbf{w}) \in \mathrm{T}\right\}$, commuting with all $\hat{S}_{Z}$ but not necessarily with each other outside of the code space. The group $\mathrm{T}$ is generated by the rotations $[(2 \pi / 3),(\mathbf{x}+\mathbf{y}+\mathbf{z}) /$ $\sqrt{3}]$ and $(\pi, \mathbf{z})$, so

$$
\begin{aligned}
& \hat{S}_{X}^{(1)}=\cos \left[\frac{2 \pi}{3 \sqrt{3}}\left(\hat{L}_{x}+\hat{L}_{y}+\hat{L}_{z}\right)\right], \\
& \hat{S}_{X}^{(2)}=(-1)^{\hat{L}_{z}},
\end{aligned}
$$

together with the $\hat{S}_{Z}$ check operator, are sufficient to identify the code space.

\section{Relation to spherical designs}

There is a one-way connection between designs and momentum-kick detection. An $L$ design is a set of points $\mathrm{P} \subset \mathrm{S}^{2}$ satisfying

$$
\int_{\mathrm{S}^{2}} d \mathbf{v} f(\mathbf{v})=\frac{1}{|\mathrm{P}|} \sum_{p \in \mathrm{P}} f(p) \equiv f(\mathrm{P})
$$

for all polynomials $f$ of degree $\ell \leq L$. An $L$ design satisfies $f(\mathrm{P})=f(R \mathrm{P})$ for any rotation $R \in \mathrm{SO}_{3}$ (see Theorem 5.6.1 in Ref. [169]). Because of this property and because spherical harmonics $\left\{Y_{m}^{\ell}\right\}$ are degree- $\ell$ polynomials restricted to the sphere, the states $|\mathrm{P}\rangle \propto \sum_{p \in \mathrm{P}}|p\rangle$ and $\hat{X}_{R}|\mathrm{P}\rangle$ form a code detecting $\leq L$ momentum kicks, where $R$ is any nontrivial rotation.

Designs often arise as orbits of a group $\mathrm{H}$ acting on a particular point $\mathbf{w}, \mathrm{P}=\mathrm{Hw}$. For example, the constituent orientations of each of the code words of our $\mathrm{T} \subset \mathrm{T} \times \mathrm{Z}_{2}^{P}$ code form a two design, and the +1 logical $X$ state consists of all points on a cube and forms a three design. (Not all of our codes are designs: The equatorial sets of points making up our $\mathrm{Z}_{N}$ code words detect momentum kicks but do not form $N-1$ designs.)

The connection to designs suggests a way to obtain other design-based codes, whose code words are not based on a single orbit or whose code words make up more complicated polyhedra [170]. There is also a potentially interesting extension of oscillator-based error-correcting codes based on designs [171] to molecular state spaces.

\section{A QUBIT ON A GROUP}

In Sec. V, we describe a family of quantum codes based on the nested subgroups $\mathrm{H} \subset \mathrm{K} \subset \mathrm{SO}_{3}$. In this section, we generalize this construction. The basic framework is already discussed in Sec. VD. We formulate quantum codes based on $\mathrm{H} \subset \mathrm{K} \subset \mathrm{G}$ using a symbolic decomposition of $\mathrm{G}$ defined by a partial Fourier transform:

$$
\mathrm{G} \cong \mathrm{G} / \mathrm{K} \times \mathrm{K} / \mathrm{H} \times \hat{\mathrm{H}} .
$$

TABLE III. List of elements of a $\mathrm{H} \subset \mathrm{K}$ code on $\mathrm{G}$ from Sec. VA. The set $\hat{H}$ consists of (equivalence classes of) all irreps of $\mathrm{H}, \mathrm{F}_{\mathrm{G} / \mathrm{K}}$ is the Voronoi cell of the identity (see Appendix B), and $\mathrm{K}^{\prime} \subset \mathrm{K}$ consists of all elements of $\mathrm{K}$ that map to identity when projected onto the logical subspace.

\begin{tabular}{lc}
\hline \hline Code & $\mathrm{H} \subset \mathrm{K} \subset \mathrm{G}$ \\
\hline Part. Fourier basis (124) & $\left\{\left|a \mathrm{H} ;{ }_{\mu \nu}^{\lambda}\right\rangle, a \in \mathrm{F}_{\mathrm{G} / \mathrm{H}, \mu \nu}^{\lambda} \in \hat{\mathrm{H}}\right\}$ \\
Logicals (126) & $\left\{\left|r \mathrm{H} ;{ }_{00}^{1}\right\rangle, r \in \mathrm{F}_{\mathrm{K} / \mathrm{H}}\right\}$ \\
Correctable position shifts & $\mathrm{F}_{\mathrm{G} / \mathrm{K}}$ \\
Correctable momentum kicks & $\mathrm{Use}$ branching formulas \\
Check operators $\hat{S}_{Z}(135)$ & $\hat{Z}_{m n}^{\ell}(\mathrm{K})$ \\
$Z$-type logicals & $\hat{Z}_{m n}^{\ell}(\mathrm{H})$ \\
Check operators $\hat{S}_{X}$ & $\left\{\vec{X}_{k} \bar{X}_{h}, k \in \mathrm{K}^{\prime}, h \in \mathrm{H}\right\}$ \\
$X$-type logicals & $\left\{\vec{X}_{k}, k \in \mathrm{K}\right\}$ \\
\hline \hline
\end{tabular}

We interpret elements of $\mathrm{G} / \mathrm{K}$ as correctable rotation errors and elements of $\hat{\mathrm{H}}$ as correctable momentum-kick errors, while elements of $\mathrm{K} / \mathrm{H}$ correspond to basis states which span the code space. Data for these codes are summarized in Table III.

We consider error-correcting codes for quantum systems whose canonical position basis $\{|g\rangle\}$ corresponds to elements of a group, $g \in \mathrm{G}$. Such spaces admit generalized versions of many of the features of standard quantum mechanical spaces such as qubits or oscillators: position and momentum bases, their corresponding shifts, orthogonality relations, a Weyl-type relation, etc. We collect these in Table IV, intending it to be an extension of an analogous table (Table 1 in Ref. [172]) for the standard spaces.

The position (momentum) bases for general $\mathrm{G}$ can be discrete (discrete) (e.g., for qudit spaces $\mathrm{G}=\mathrm{Z}_{D}^{\times n}$ ), continuous (discrete) (for rotors $\mathrm{G} \in\left\{\mathrm{U}_{1}, \mathrm{SO}_{3}\right\}$ ), or continuous (continuous) (for oscillators $\mathrm{G}=\mathrm{R}$ ). These differences obscure the intuition we are trying to convey, so we keep $\mathrm{G}$ finite for clarity here. The caption of Table IV adapts these discussions to other G, and Ref. [127] rigorously formulates many of the required tools for type-I unimodular second-countable groups.

Most of the structure for general $\mathrm{G}$ is already present for $\mathrm{G}=\mathrm{SO}_{3}$, which we outline in Sec. V. Position shifts $\vec{X}$ for general G are represented by left multiplication, $\vec{X}_{h}|g\rangle=$ $|h g\rangle$, and analogous shifts $\overleftarrow{X}$ exist for right multiplication. "Momentum" kick operators are diagonal in position space, acting as [175-177]

$$
\hat{Z}_{m n}^{\ell}|g\rangle=Z_{m n}^{\ell}(g)|g\rangle,
$$

where $Z_{m n}^{\ell}(g)$ is the $m, n$th matrix element of the group element $\mathrm{G}$ in the irrep $\ell$. These matrix elements are part of the momentum basis for $\mathrm{G}$ : 
TABLE IV. Summary of relations for $\mathcal{L}^{2}(\mathrm{G})$ - the space of $\mathcal{L}^{2}$-normalizable functions on a group G-extending analogous summaries for ordinary qudit and oscillator state spaces (see Table 1 in Ref. [172]). The $C_{\ell m \ell^{\prime} m^{\prime}}^{L M}$ are Clebsch-Gordan coefficients [109,153]. When $\mathrm{G}=\mathrm{Z}_{D}^{\times n}$, the state space is that of $n$ qudits, and the position states and their corresponding momentum states are both discrete orthonormal bases. The rotor state spaces $\mathrm{U}_{1}$ and $\mathrm{SO}_{3}$ and, more generally, any continuous compact $\mathrm{G}$ admit bases of position states in the continuous or Dirac sense (see Sec. VI. 6 in Ref. [173]). In those cases, $(1 /|\mathrm{G}|) \sum_{g \in \mathrm{G}}$ is replaced by $(1 /|\mathrm{G}|) \int_{\mathrm{G}} d g$, where $|\mathrm{G}|$ is the volume of $\mathrm{G}$ as a manifold and $d g$ is the Haar measure [154]. However, since such spaces are compact, their corresponding momentum bases are still discrete. The oscillator $\mathrm{G}=\mathrm{R}$ is continuous and noncompact, meaning that both its position and momentum bases are continuous. For this group and others like it, $1 /|\mathrm{G}|$ is omitted, and the sum over $\ell$ turns into an integral with respect to the Plancherel measure $d \ell$ [174] (see also Sec. 8.3.3 in Ref. [154]).

\begin{tabular}{|c|c|c|}
\hline & Finite group $\mathcal{L}^{2}(\mathrm{G})$ & Rigid rotor $\mathcal{L}^{2}\left(\mathrm{SO}_{3}\right)$ \\
\hline A. "Phase space" & $\left(g,{ }_{m n}^{\ell}\right) \in \mathrm{G} \times \hat{\mathrm{G}}$ & $\left(R,,_{m n}^{\ell}\right) \in \mathrm{SO}_{3} \times \widehat{\mathrm{SO}_{3}}$ \\
\hline B. Conjugate bases & $\begin{array}{l}\left.|g\rangle=\left.\sum_{\ell m n \in \hat{\mathrm{G}}} \sqrt{\left(d_{\ell} /|\mathrm{G}|\right)} Z_{m n}^{\ell \star}(g)\right|_{m n} ^{\ell}\right\rangle \\
\left|{ }_{m n}^{\ell}\right\rangle=\sum_{g \in \mathrm{G}} \sqrt{\left(d_{\ell} /|\mathrm{G}|\right)} Z_{m n}^{\ell}(g)|g\rangle\end{array}$ & $\begin{array}{c}\left.|R\rangle=\left.\sum_{\ell \geq 0} \sum_{|m|,|n| \leq \ell} \sqrt{\left(2 \ell+1 / 8 \pi^{2}\right)} D_{m n}^{\ell \star}(R)\right|_{m n} ^{\ell}\right\rangle \\
\left|{ }_{m n}^{\ell}\right\rangle=\int_{\mathrm{SO}_{3}} d R \sqrt{\left(2 \ell+1 / 8 \pi^{2}\right)} D_{m n}^{\ell}(R)|R\rangle\end{array}$ \\
\hline C. Overlap & $\left\langle\left. g\right|_{m n} ^{\ell}\right\rangle=\sqrt{\left(d_{\ell} /|\mathrm{G}|\right)} Z_{m n}^{\ell}(g)$ & $\left\langle\left. R\right|_{m n} ^{\ell}\right\rangle=\sqrt{\left(2 \ell+1 / 8 \pi^{2}\right)} D_{m n}^{\ell}(R)$ \\
\hline D. "Resolution" & $\sum_{g \in \mathrm{G}}|g\rangle\left\langle g\left|=\sum_{\ell m n \in \hat{\mathrm{G}}}\right|{ }_{m n}^{\ell}\right\rangle\left\langle{ }_{m n}^{\ell}\right|=1_{\mathrm{G}}$ & $\int_{\mathrm{SO}_{3}} d R|R\rangle\left\langle\left. R\left|=\sum_{\ell \geq 0} \sum_{|m|,|n| \leq \ell}\right|\right|_{m n} ^{\ell}\right\rangle\left\langle{ }_{m n}^{\ell}\right|=1_{\mathrm{SO}_{3}}$ \\
\hline E. "Orthocompleteness" & $\begin{array}{c}\sum_{g \in \mathrm{G}} Z_{m n}^{\ell \star}(g) Z_{m^{\prime} n^{\prime}}^{\ell^{\prime}}(g) \\
=\left(|\mathrm{G}| / d_{\ell}\right) \delta_{\ell \ell^{\prime}} \delta_{m m^{\prime}} \delta_{n n^{\prime}} \\
\sum_{\ell m n \in \hat{\mathrm{G}}}\left(d_{\ell} /|\mathrm{G}|\right) Z_{m n}^{\ell \star}(g) Z_{m n}^{\ell}\left(g^{\prime}\right)=\delta_{g g^{\prime}}^{\mathrm{G}}\end{array}$ & $\begin{array}{c}\int_{\mathrm{SO}_{3}} d R D_{m n}^{\ell \star}(R) D_{m^{\prime} n^{\prime}}^{\ell^{\prime}}(R) \\
=\left(8 \pi^{2} / 2 \ell+1\right) \delta_{\ell \ell^{\prime}} \delta_{m m^{\prime}} \delta_{n n^{\prime}} \\
\sum_{\ell \geq 0} \sum_{|m|,|n| \leq \ell}\left(2 \ell+1 / 8 \pi^{2}\right) \\
D_{m n}^{\ell \star}(R) D_{m n}^{\ell}\left(R^{\prime}\right)=\delta_{R R^{\prime}}^{\mathrm{SO}_{3}}\end{array}$ \\
\hline F. Position shifts & $\begin{array}{c}\vec{X}_{h}|g\rangle=|h g\rangle \\
\overleftarrow{X}_{h}|g\rangle=\left|g h^{-1}\right\rangle \\
\left.\left.\left.\vec{X}_{h}\right|_{m n} ^{\ell}\right\rangle=\left.\sum_{p} Z_{p m}^{\ell \star}(h)\right|_{p n} ^{\ell}\right\rangle \\
\left.\left.\left.\overleftarrow{X}_{h}\right|_{m n} ^{\ell}\right\rangle=\left.\sum_{p} Z_{p n}^{\ell}(h)\right|_{m p} ^{\ell}\right\rangle\end{array}$ & $\begin{array}{c}\vec{X}_{S}|R\rangle=|S R\rangle \\
\overleftarrow{X}_{S}|R\rangle=\left|R S^{-1}\right\rangle \\
\left.\left.\vec{X}_{R}||_{m n}^{\ell}\right\rangle=\left.\sum_{p} D_{p m}^{\ell \star}(R)\right|_{p n} ^{\ell}\right\rangle \\
\left.\overleftarrow{X}_{R}\left|{ }_{m n}^{\ell}\right\rangle=\left.\sum_{p} D_{p n}^{\ell}(R)\right|_{m p} ^{\ell}\right\rangle\end{array}$ \\
\hline G. Momentum kicks & $\begin{array}{c}\hat{Z}_{m n}^{\ell}|g\rangle=Z_{m n}^{\ell}(g)|g\rangle \\
\left.\left.\hat{Z}_{m n}^{\ell}||_{m^{\prime} n^{\prime}}^{\ell^{\prime}}\right\rangle=\left.\sum_{L M N \in \hat{\mathrm{G}}} c_{\ell m n, \ell^{\prime} m^{\prime} n^{\prime}}^{L M N}\right|_{M N} ^{L}\right\rangle \\
c_{\ell m n, \ell^{\prime} m^{\prime} n^{\prime}}^{L M}=\left(\sqrt{d_{\ell^{\prime}} d_{L}} /|\mathrm{G}|\right) \\
\sum_{g \in \mathrm{G}} Z_{M N}^{L \star}(g) Z_{m n}^{\ell}(g) Z_{m^{\prime} n^{\prime}}^{\ell^{\prime}}(g)\end{array}$ & $\begin{array}{c}\hat{D}_{m n}^{\ell}|R\rangle=D_{m n}^{\ell}(R)|R\rangle \\
\left.\left.\hat{D}_{m n}^{\ell}||_{m^{\prime} n^{\prime}}^{\ell^{\prime}}\right\rangle=\left.\sum_{L \geq 0} \sum_{|M|,|N| \leq L} c_{\ell m n, \ell^{\prime} m^{\prime} n^{\prime}}^{L M N}\right|_{M N} ^{L}\right\rangle \\
c_{\ell m n, \ell^{\prime} m^{\prime} n^{\prime}}^{L M N}=\sqrt{\left(2 \ell^{\prime}+1\right) /(2 L+1)} C_{\ell m \ell^{\prime} m^{\prime}}^{L M} C_{\ell n \ell^{\prime} n^{\prime}}^{L N}\end{array}$ \\
\hline H. "Weyl relation" & $\begin{aligned} \vec{X}_{g} \hat{Z}_{m n}^{\ell} \vec{X}_{g}^{\dagger} & =\sum_{p} Z_{p m}^{\ell \star}(g) \hat{Z}_{p n}^{\ell} \\
\overleftarrow{X}_{g} \hat{Z}_{m n}^{\ell} \overleftarrow{X}_{g}^{\dagger} & =\sum_{p} Z_{p n}^{\ell}(g) \hat{Z}_{m p}^{\ell}\end{aligned}$ & $\begin{aligned} \vec{X}_{R} \hat{D}_{m n}^{\ell} \vec{X}_{R}^{\dagger} & =\sum_{p} D_{p m}^{\ell \star}(R) \hat{D}_{p n}^{\ell} \\
\overleftarrow{X}_{R} \hat{D}_{m n}^{\ell} \overleftarrow{X}_{R}^{\dagger} & =\sum_{p} D_{p n}^{\ell}(R) \hat{D}_{m p}^{\ell}\end{aligned}$ \\
\hline
\end{tabular}

$$
\left.\left|{ }_{m n}^{\ell}\right\rangle=\sum_{g \in \mathrm{G}} \sqrt{\frac{d_{\ell}}{|\mathrm{G}|}} Z_{m n}^{\ell}(g)|g\rangle=\left.\sqrt{d_{\ell}} \hat{Z}_{m n}^{\ell}\right|_{00} ^{\mathbf{1}}\right\rangle .
$$

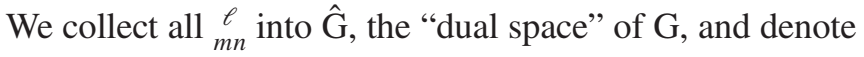
the trivial irrep as $\lambda=\mathbf{1}$.

Products of position shifts and momentum kicks, $\hat{B}_{g}^{\ell m n}=\sqrt{\left(d_{\ell} /|\mathrm{G}|\right)} \hat{Z}_{m n}^{\ell} \vec{X}_{g}$, form an orthonormal and complete basis for operators on $\mathrm{G}$ :

$$
\begin{array}{r}
\operatorname{Tr}\left(\hat{B}_{g}^{\ell m n n^{\dagger}} \hat{B}_{g^{\prime}}^{\ell^{\prime} m^{\prime} n^{\prime}}\right)=\delta_{g g^{\prime}}^{\mathrm{G}} \delta_{\ell \ell^{\prime}} \delta_{m m^{\prime}} \delta_{n n^{\prime}}, \\
\sum_{g \in \mathrm{G}} \sum_{\ell m n \in \hat{\mathrm{G}}}\left\langle h\left|\hat{B}_{g}^{\ell m n}\right| k\right\rangle\left\langle k^{\prime}\left|\hat{B}_{g}^{\ell m n^{\dagger}}\right| h^{\prime}\right\rangle=\delta_{h h^{\prime}}^{\mathrm{G}} \delta_{k k^{\prime}}^{\mathrm{G}},
\end{array}
$$

where $\operatorname{Tr}(\cdot)=\sum_{g \in \mathrm{G}}\langle g|(\cdot)| g\rangle$ and $h, h^{\prime}, k, k^{\prime} \in \mathrm{G}$. Thus, any physical noise channel $\mathcal{E}$ acting on this space can be expanded in terms of this operator basis, as before [Eqs. (9) and (42)]. The purpose of our codes is to protect against "small" position shifts as well as certain momentum shifts.

\section{A. Partial Fourier transform}

Our code constructions make use of the partial Fourier transform on $\mathrm{G}$, whose states are parameterized by cosets in $\mathrm{G} / \mathrm{H}$ and $\mathrm{H}$ irreps:

$$
\left|a \mathrm{H} ;{ }_{\mu \nu}^{\lambda}\right\rangle=\sqrt{\frac{d_{\lambda}}{|\mathrm{H}|}} \sum_{h \in \mathrm{H}} \mathrm{Z}_{\mu \nu}^{\lambda}(h)|a h\rangle .
$$

Above, $a$ belongs to the coset space $\mathrm{G} / \mathrm{H}$, which we parameterize using $\mathrm{F}_{\mathrm{G} / \mathrm{H}}$, the Voronoi cell of the identity (see Appendix B). The coefficient $Z_{\mu \nu}^{\lambda}(h)$ is the $\mu, \nu$ th matrix element of the $d_{\lambda}$-dimensional irrep $\lambda$ of $\mathrm{H}$, evaluated for the element $h \in \mathrm{H}$. We use the Greek letters $\lambda$, $\mu$, and $\nu$ to label matrix elements of irreps of $\mathrm{H}$ and save 
the letters $\ell, m$, and $n$ for labeling matrix elements of irreps of $\mathrm{G}$.

The above basis interpolates between the group's position states $(\mathrm{H}=\{1\})$ and momentum states $(\mathrm{H}=\mathrm{G})$. One can show that it is orthonormal and complete [178]:

$$
\begin{array}{r}
\left\langle a \mathrm{H} ;{ }_{\mu \nu}^{\lambda} \mid a^{\prime} \mathrm{H} ; \mu_{\mu^{\prime} \nu}^{\lambda^{\prime}}\right\rangle=\delta_{a a^{\prime}}^{\mathrm{G} / \mathrm{H}} \delta_{\lambda \lambda^{\prime}} \delta_{\mu \mu^{\prime}} \delta_{\nu \nu^{\prime}}, \\
\sum_{a \in \mathrm{G} / \mathrm{H}} \sum_{\lambda \mu \nu \in \hat{\mathrm{H}}}\left\langle g \mid a \mathrm{H} ;{ }_{\mu \nu}^{\lambda}\right\rangle\left\langle a \mathrm{H} ;{ }_{\mu \nu}^{\lambda} \mid g^{\prime}\right\rangle=\delta_{g g^{\prime}}^{\mathrm{G}} .
\end{array}
$$

This basis arises in several other contexts in science and engineering, which we discuss in Appendix F.

\section{B. Code words}

Our code words correspond to cosets of $\mathrm{H}$ in $\mathrm{K}$. For $r \in \mathrm{F}_{\mathrm{K} / \mathrm{H}}$,

$$
|\bar{r}\rangle \equiv\left|r \mathrm{H} ;{ }_{00}^{1}\right\rangle=\frac{1}{\sqrt{|\mathrm{H}|}} \sum_{h \in \mathrm{H}}|r h\rangle .
$$

Expressing the G position states (126) in terms of momentum states yields

$$
\left.|\bar{r}\rangle=\left.\sum_{\ell m n \in \hat{\mathrm{G}}} \sqrt{\frac{d_{\ell}}{|\mathrm{G} / \mathrm{H}|}} Z_{m n}^{\ell \star}(r \mathrm{H})\right|_{m n} ^{\ell}\right\rangle .
$$

Here, we introduce the notation $f(\mathrm{H})$ for the $H$ average (also called $H$ twirl) of a function $f$ on $\mathrm{G}$ over the subgroup $\mathrm{H}$, defined by

$$
f(\mathrm{H}) \equiv \frac{1}{|\mathrm{H}|} \sum_{h \in \mathrm{H}} f(h) .
$$

Observing that $Z_{m n}^{\ell \star}(r \mathrm{H})=\left[\mathrm{Z}^{\ell \star}(r) Z^{\ell \star}(\mathrm{H})\right]_{m n}$, we see that the momentum state $\left|{ }_{m n}^{\ell}\right\rangle$ "participates" in the expansion of $|\bar{r}\rangle$ (occurs with a nonzero coefficient) only if $Z^{\ell}(\mathrm{H}) \neq 0$. Irreps with this property make up the reciprocal space of $\mathrm{H}$ in $\mathrm{G}$ :

$$
\mathrm{H}^{\perp} \equiv\left\{\ell \in \hat{\mathrm{G}}, Z^{\ell}(\mathrm{H}) \neq 0\right\} .
$$

For each $\ell$, we also have to determine the participating $m, n$ indices; these depend on the basis used for $Z^{\ell}$ (see Appendix D).

Denoting the set of participating momentum-state indices $\underset{m n}{\ell}$ by $\widehat{\mathrm{G} / \mathrm{H}}$, Eq. (127) becomes

$$
\left.|\bar{r}\rangle=\left.\sum_{\ell m n \in \widehat{\mathrm{G} / \mathrm{H}}} \sqrt{\frac{d_{\ell}}{|\mathrm{G} / \mathrm{H}|}} Z_{m n}^{\ell \star}(r \mathrm{H})\right|_{m n} ^{\ell}\right\rangle .
$$

We thus map the position degree of freedom $r$ from the ket $|r h\rangle$ of the position-basis expansion (126) into the coefficient $Z_{m n}^{\ell \star}(r \mathrm{H})$ of the momentum-basis expansion (130). This mapping can be done for any coset state $\left|a \mathrm{H} ;{ }_{00}^{1}\right\rangle$ with $a \in \mathrm{G} / \mathrm{H}-\mathrm{a}$ manifestation of the Fourier transform on $\mathrm{G} / \mathrm{H}$ (see Appendix D). Analogously, one can develop a Fourier transform on the code space $\mathrm{K} / \mathrm{H}$.

\section{Position shifts}

Position shifts acting from the left realize an induced representation for each $\lambda$ in Eq. (124), meaning that $\left\{\vec{X}_{g}\right\}_{g \in \mathrm{G}}$ do not connect different $\lambda$ 's. The difference from the Abelian case (50) is the behavior of the internal indices $\mu \nu$ :

$$
\vec{X}_{g}\left|a \mathrm{H} ;{ }_{\mu \nu}^{\lambda}\right\rangle=\sum_{\rho} Z_{\rho \mu}^{\lambda \star}\left(k_{g}\right)\left|g a k_{g}^{-1} \mathrm{H}_{\rho \nu}^{\lambda}\right\rangle .
$$

Above, the compensating element $k_{g} \in \mathrm{H}$ is picked such that $\operatorname{gak}_{g}^{-1} \in \mathrm{F}_{\mathrm{G} / \mathrm{H}}$.

Let us determine the set of correctable position shifts $\vec{X}_{g}$. First, consider $g \in \mathrm{F}_{\mathrm{G} / \mathrm{K}}$, in which case there is no compensating element. Then, the error state obtained from applying a momentum kick and position shift to the code word $|\vec{r}\rangle$ consists of a superposition of the basis elements (124) with $a=g r$. By measuring a rotation in $\mathrm{F}_{\mathrm{G} / \mathrm{K}}$ and applying the corresponding position shift, the recovery maps each $g r$ to the element $r^{\prime} \in \mathrm{K} / \mathrm{H}$ whose Voronoi cell contains $g r$. (The partitioning into cosets ensures that the Voronoi cell of each $r^{\prime} \in \mathrm{K} / \mathrm{H}$ contains only one $g r$.) Since $g \in \mathrm{F}_{\mathrm{G} / \mathrm{K}}, g r$ are in the Voronoi cell of $r$, and so $r^{\prime}=r$. After recovery, each $r$ returns to its original location.

Now, consider $g \notin \mathrm{F}_{\mathrm{G} / \mathrm{K}}$. Now, the corrupted position label corresponding to code word $r$ can stray into the Voronoi cell of some other element $r^{\prime} \neq r$. The above recovery snaps such error words to the wrong code words, leading to logical errors. In the case of non-Abelian codes, there may be additional errors due to the effect of the compensating element on $\mu \nu$ (131).

\section{Momentum kicks}

Assuming we exactly correct a position shift, the resulting state lies in the span of $\left\{\left|r \mathrm{H} ;{ }_{\mu \nu}^{\lambda}\right\rangle\right\}$ for all $r \in$ $\mathrm{K} / \mathrm{H}$ and ${ }_{\mu \nu}^{\lambda} \in \hat{\mathrm{H}}$. Below, we show how to use the branching formulas for $\mathrm{G}$ restricted to $\mathrm{K}$, and then $\mathrm{K}$ restricted to $\mathrm{H}$, to determine detectable and correctable momentum kicks $\hat{Z}_{m n}^{\ell}$. We leave the precise formulation of a momentumkick recovery for general $\mathrm{G}$ to future work.

Let $\ell \in \hat{\mathrm{G}}, \kappa \in \hat{\mathrm{K}}$, and $\lambda \in \hat{\mathrm{H}}$, so that $\ell \rightarrow \kappa \rightarrow \lambda$ means that $\ell$ contains at least one copy of the $\mathrm{K}$ irrep $\kappa$ when restricted to $\mathrm{K}$, which, in turn, contains at least one copy of $\lambda$ when restricted to $H$. We denote the trivial irrep by $\mathbf{1}$. For convenience, we assume that $Z^{\ell}$ are written in a $\mathrm{K}$ admissible basis (see Appendix D), meaning that $Z^{\ell}(k)$ for $k \in \mathrm{K}$ are block diagonal with respect to the $\mathrm{K}$ irreps. 
Similarly, we assume that those $Z^{\ell}(k)$ are, in turn, in an $\mathrm{H}$ admissible basis.

First, consider detectable errors. Let $\ell$ be such that one of its branches is $\ell \rightarrow \kappa \rightarrow \mathbf{1}$ with $\kappa \neq \mathbf{1}$. Then, there exists a momentum kick $\hat{Z}_{m n}^{\ell}$ for some $m, n$ that is undetectable. To prove this statement, consider matrix-valued versions of the error-correction criteria, projecting the matrix of G-momentum shifts $\hat{Z}^{\ell}$ into the code space (126):

$$
\left\langle\bar{r}\left|\hat{Z}^{\ell}\right| \bar{r}\right\rangle=Z^{\ell}(r \mathrm{H})=Z^{\ell}(r) Z^{\ell}(\mathrm{H}) .
$$

We show that the above depends on $r$.

Since $Z^{\ell}(h)$ is in an $\mathrm{H}$-admissible basis, we can express each $Z^{\ell}(h)$ in $Z^{\ell}(\mathrm{H}) \propto \sum_{h \in \mathrm{H}} Z^{\ell}(h)$ as a direct sum of irreps of $\mathrm{H}$. By the group orthogonality relations on $\mathrm{H}$, $Z_{m n}^{\ell}(\mathrm{H})=\delta_{m n}$ only for those $n$ which correspond to matrix elements of $\mathrm{H}$ in the trivial $\mathrm{H}$ irrep. We assume that $\ell$ branches to at least one trivial irrep of $\mathrm{H}$, so there exists such an $n$, which we call $n_{\star}$. [This assumption implies that $\ell \in \mathrm{H}^{\perp}$ (129), a necessary but not sufficient condition for undetectability.] Now, consider the column $Z_{m n_{\star}}^{\ell}(r \mathrm{H})$, with $m \in\left\{1,2, \ldots, d_{\kappa}\right\}$ and $d_{\kappa}$ being the dimension of the $\kappa$ irrep that contains the trivial irrep $\lambda=\mathbf{1}$. When $r=1$ (the identity), $Z_{m n}^{\ell}(r \mathrm{H})=\delta_{m n}$. But, since $\kappa \neq \mathbf{1}$, there exists another $r^{\prime} \neq 1$ that is represented differently. Thus, $Z_{m n}^{\ell}\left(r^{\prime} \mathrm{H}\right) \neq \delta_{m n}$, and Eq. (132) depends on $r$.

Now, consider correctable errors. Let $\ell \neq \ell^{\prime}$ be such that they branch to the same nontrivial $\mathrm{H}$ irrep via different nontrivial $\mathrm{K}$ irreps $\kappa \neq \kappa^{\prime}$, i.e., $\ell \rightarrow \kappa \rightarrow \lambda$ and $\ell^{\prime} \rightarrow \kappa^{\prime} \rightarrow \lambda$, respectively. Then, momentum kicks $\hat{Z}^{\ell}$ and $\hat{Z}^{\ell^{\prime}}$ are not simultaneously correctable. To prove this result, let $m, n$ be the matrix elements of the copy of $\lambda$ contained in $\kappa$ and $m^{\prime}$, $n^{\prime}$ be those for the copy of $\lambda$ contained in $\kappa^{\prime}$. Then,

$$
\begin{gathered}
\hat{Z}_{m n}^{\ell}|\bar{r}\rangle=\frac{1}{\sqrt{|\mathrm{H}|}} \sum_{h \in \mathrm{H}} \sum_{p=1}^{d_{\lambda}} Z_{m p}^{\kappa}(r) Z_{p n}^{\lambda}(h)|r h\rangle \\
=\frac{1}{\sqrt{d_{\lambda}}} \sum_{p=1}^{d_{\lambda}} Z_{m p}^{\kappa}(r)\left|r \mathrm{H} ;{ }_{p n}\right\rangle,
\end{gathered}
$$

where we use the basis (124) in the second line. Using orthogonality of this basis,

$$
\left\langle\bar{r}\left|\hat{Z}_{m^{\prime}, n^{\prime}}^{\ell^{\prime} \dagger} \hat{Z}_{m, n}^{\ell}\right| \bar{r}\right\rangle=\frac{\delta_{n n^{\prime}}}{d_{\lambda}} \sum_{p=1}^{d_{\lambda}} Z_{m p}^{\kappa}(r) Z_{p m^{\prime}}^{\kappa^{\prime}}\left(r^{-1}\right) .
$$

When $r=1$, the sum over $p$ reduces to $\delta_{m m^{\prime}}$. But, since $\kappa \neq \kappa^{\prime}$, there exists an $r^{\prime} \neq 1$ such that the above yields a different result. Therefore, one cannot correct both $\ell$ and $\ell^{\prime}$ momentum kicks.

\section{E. Gates and check operators}

Logical $X$-type gates include all $\left\{\vec{X}_{k}\right\}_{k \in \mathrm{K}}$, which realize an induced representation on the logical subspace $\mathrm{K} / \mathrm{H}$. A subset of those, which we call $\mathrm{K}^{\prime} \subset \mathrm{K}$, acts as the identity in this induced representation; such operators can be used as check operators for momentum-kick syndrome measurement. Examples of such representations are discussed in Sec. V E.

The position shifts $\left\{\overleftarrow{X}_{h}\right\}_{h \in \mathrm{H}}$ also act trivially on the code words. These do not commute with each other for nonAbelian $\mathrm{H}$ but do commute with $\vec{X}_{k}$ (since left and right multiplication commute). These can also be used as check operators, and the resulting combined set of $X$-type check operators is listed in Table III.

If $\mathrm{H}$ is a normal subgroup of $\mathrm{K}$, then the shifts $\left\{\vec{X}_{k}\right\}_{k \in \mathrm{K}}$ also realize logical gates; otherwise, such shifts may not preserve the code subspace (since left and right coset spaces, $\mathrm{K} / \mathrm{H}$ and $\mathrm{H} \backslash \mathrm{K}$, are not equal). For $\mathrm{G}=\mathrm{SO}_{3}$, such cases include $\mathrm{Z}_{N} \subset \mathrm{Z}_{2 N}$ and $\mathrm{T} \subset \mathrm{O}$ but not $\mathrm{T} \subset \mathrm{I}$.

Twirling momentum-kick operators over $\mathrm{K}$,

$$
\hat{Z}_{m n}^{\ell}(\mathrm{K}) \equiv \frac{1}{|\mathrm{~K}|} \sum_{k \in \mathrm{K}} \overleftarrow{X}_{k} \hat{Z}_{m n}^{\ell} \overleftarrow{X}_{k}^{\dagger}
$$

offers a convenient method for generating $Z$-type check operators $\hat{S}_{Z}$. The above operators are functions on $\mathrm{G} / \mathrm{K}$ :

$$
\hat{Z}_{m n}^{\ell}(\mathrm{K})\left|w r \mathrm{H} ;{ }_{\mu \nu}^{\lambda}\right\rangle=Z_{m n}^{\ell}(w \mathrm{~K})\left|w r \mathrm{H} ;{ }_{\mu \nu}^{\lambda}\right\rangle
$$

for $w \in \mathrm{G} / \mathrm{K}$ and $r \in \mathrm{K} / \mathrm{H}$. Thus, measuring them does not spoil the logical information. A projective measurement onto the basis of the joint eigenstates of these mutually commuting operators can be used to determine the syndrome $w$. (The scheme outlined below implicitly performs such a measurement.) Such $\hat{S}_{Z}$ commute with each other and all $\left\{\overleftarrow{X}_{k}\right\}_{k \in \mathrm{K}}$ but commute with $\left\{\vec{X}_{k}\right\}_{k \in \mathrm{K}}$ only on the subspace $\left\{\left|r \mathrm{H} ;{ }_{\mu \nu}^{\lambda}\right\rangle\right\}$ with $r \in \mathrm{K} / \mathrm{H}$ and ${ }_{\mu \nu}^{\lambda} \in \hat{\mathrm{H}}$. Since they can be nonunitary, they do not, in general, form a group.

Twirling momentum kicks over $\mathrm{H}$ produces logical Ztype operators. A similar procedure yields $Z$-type check and logical operators for codes on $S^{2}$ (see Appendix E).

To construct a code Hamiltonian, recall that the code states (126) are equal superpositions of elements of cosets of $\mathrm{H}$ in $\mathrm{K}$. In other words, a Hamiltonian (3) whose ground states form the code space can be a projection onto those cosets of $\mathrm{H}$ in $\mathrm{G}$ which consist of elements in K. Such a Hamiltonian is then

$$
H_{\text {code }}=-\sum_{k \in \mathrm{K}}|k\rangle\left\langle k\left|-\sum_{a \in \mathrm{G} / \mathrm{H}}\right| a \mathrm{H}\right\rangle\langle a \mathrm{H}|,
$$

where $|a \mathrm{H}\rangle \equiv\left|a \mathrm{H} ;{ }_{00}^{\mathbf{1}}\right\rangle$. The second term projects onto $\mathrm{G} / \mathrm{H}$, while the first picks out only the coset states with elements in K. Using twirled momentum kicks and the group orthogonality relations, one can construct the projection onto the subgroup as 


$$
\sum_{k \in \mathrm{K}}|k\rangle\langle k|=\sum_{\ell, m \in \hat{\mathrm{G}}} \frac{d_{\ell}}{|\mathrm{G} / \mathrm{K}|} \hat{Z}_{m m}^{\ell}(\mathrm{K}) .
$$

The position shifts can be used to construct the projection onto $\mathrm{G} / \mathrm{H}$ (see Sec. VII C in Ref. [179]):

$$
\sum_{a \in \mathrm{G} / \mathrm{H}}|a \mathrm{H}\rangle\langle a \mathrm{H}|=\frac{1}{|\mathrm{H}|} \sum_{h \in \mathrm{H}} \overleftarrow{X}_{h} .
$$

\section{F. Measurements}

Recall that, given a subgroup $\mathrm{K}$, each group element $g \in \mathrm{G}$ can be written as $g=a k$ for $a \in \mathrm{F}_{\mathrm{G} / \mathrm{K}}$ and $k \in \mathrm{K}$. In order to diagnose which position shift occurs without destroying the logical information, one needs to read off the coset label $a$ without obtaining information about K. Since there are only $|\mathrm{G} / \mathrm{K}|$ different values one needs to distinguish, we can pick the ancillary space to be $\mathrm{G} / \mathrm{K}$ (instead of the larger $\mathrm{G}$ ) and still measure in one shot.

Letting $\hat{X}_{g}$ be the induced representation of $\mathrm{G}$ on $\mathrm{G} / \mathrm{K}$, we apply the generalized CROT gate (cf. [175])

$$
\mathrm{CROT}_{\mathrm{G} / \mathrm{K}}=\sum_{g \in \mathrm{G}}|g\rangle\langle g| \otimes \hat{X}_{g}
$$

onto the $\mathrm{G}$ space housing our logical information and an ancilla initialized in some state $|\mathrm{K}\rangle$ (assumed invariant under $\left\{\hat{X}_{k}\right\}_{k \in \mathrm{K}}$ ). Since $\hat{X}_{g}=\hat{X}_{a} \hat{X}_{k}$ and since the initial state is $\mathrm{K}$ invariant, the ancilla obtains only the coset label $a$, without destroying coherences between elements of the coset. This procedure can also be used for logical state initialization.

The space G/K can be "simulated" by a finite-dimensional space spanned by generalized spin-coherent states [99], similar to our construction from Sec. V B.

\section{CONCLUSION AND FUTURE WORK}

We have developed error-correcting codes that protect against small shifts in the position and momentum of a rigid body and, more generally, of a state space $\{|g\rangle, g \in \mathrm{G}\}$ where $\mathrm{G}$ is a group. Our treatment unifies CSS codes $\left(\mathrm{G}=\mathrm{Z}_{D}^{\times n}\right)$ with GKP codes for qudits $\left(\mathrm{G}=\mathrm{Z}_{N}=\mathrm{C}_{N}\right)$, oscillators $(\mathrm{G}=\mathrm{R})$, and planar rotors $\left(\mathrm{G}=\mathrm{U}_{1}=\mathrm{SO}_{2}=\mathrm{C}_{\infty}\right.$ or $\mathrm{G}=\mathrm{Z}$ ). We propose using our rigid-body codes, for which $\mathrm{G}$ is the three-dimensional proper rotation group $\mathrm{SO}_{3}$, to robustly encode quantum information in the rotational states of asymmetric molecules.

We also constructed related codes that protect a linear rotor, whose configuration space $S^{2}$ is a coset space rather than a group, and we formulated position and momentum bases, their associated shifts, and orthogonality relations for general coset spaces.

A basis may be chosen for a rigid-body code space such that each basis state is a uniform superposition of a finite number of possible orientations for the body. Because position eigenstates in a continuous-variable system are not normalizable, the ideal code words are likewise not normalizable and have infinite energy. But we may instead choose normalizable, finite-energy approximate code words which retain good error-correction properties.

Our coding scheme has potential applications to polar molecules, certain spin systems, atomic ensembles, single atoms, and levitated nanoparticles. We now mention several possible topics for future investigation.

\section{A. Physical noise}

Our codes are designed to protect against noise that acts "locally in phase space." For CSS codes, the correctable errors are low-weight Pauli operators acting on a few qubits. For GKP oscillator codes, the correctable errors are small shifts in the position or momentum of the oscillator. For rigid-rotor codes, the correctable errors are small shifts in the rotor's orientation or small kicks in its angular momentum.

Physical noise may act nonlocally in phase space. But it has recently been shown that the dominant noise in microwave cavities is sufficiently local for GKP codes to work effectively $[3,4]$. It remains to be seen whether the noise in realistic rigid rotors [146-152] has similarly benign properties.

\section{B. Metrology}

The geometry of $\mathrm{SO}_{3}$ - the configuration space of molecular orientations-is uniquely suited for sensing three-dimensional rotations. Moreover, since such rotations are parameterized by an axis and an angle, the ability to measure them corresponds to the ability to resolve the direction and magnitude of three-dimensional vectors. Thus, one can sense three-dimensional vectors using one molecule, which is less resource intensive than, e.g., using three harmonic oscillators.

Encoding one "logical" $\mathrm{SO}_{3}$ space into several physical spaces provides an additional layer of protection, maintaining a delicate balance between noise resilience and the ability to extract the signal. Combined with recent work (by some of us) studying such encodings [180], our framework may pave the way for schemes using entangled molecular rotational or nuclear states for noise-resilient sensing. It would also be interesting to extend Ref. [181] and develop codes protecting a generalized continuous space against continuous errors.

\section{Symmetric molecules}

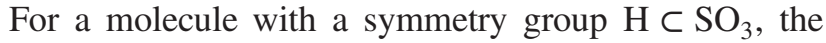
configuration space of molecular orientations is the coset space $\mathrm{SO}_{3} / \mathrm{H}$. A larger symmetry group means a smaller configuration space and, thus, less room for diagnosing rotation errors. In the extreme case of a perfect sphere, invariant under any $\mathrm{SO}_{3}$ rotation, there is no room in the onedimensional space $\mathrm{SO}_{3} / \mathrm{SO}_{3}$ for any logical information at all. It would be interesting to investigate further how the 
performance of our codes depends on the symmetry group. Of particular interest are $\mathrm{Z}_{3}$-symmetric molecules (such as monomethoxides [26] or Posner molecules [124,125]), which are invariant under rotations by $\pm 120^{\circ}$.

\section{Nuclear motion}

Instead of considering rigid molecules that are assumed to be in a fixed vibrational state, one can also consider "floppy" molecules for which there is no clear separation between rotational and vibrational motion. Such motion ranges from small nuclear vibrations around equilibrium positions [60] to larger-scale bending motion [182] and even nuclear permutations [183]. To devise codes that protect quantum information carried by (for example) floppy molecules, we need to consider different configuration spaces than for the rigid-body codes described here. Nevertheless, some of the mathematical tools we have developed may be applicable in this broader context.

\section{E. General groups}

We focus on groups applicable to molecular physics, but our general framework for a group $\mathrm{G}$ yields codes for any physically reasonable groups. One could consider $\mathrm{G}=\mathrm{S}_{n}$, the permutation group of $n$ distinct objects; such codes could be used to store quantum information in distinguishable particles or to generalize classical codes for transposition errors [184]. Another example is $G=E_{2}$, the Euclidean group of two-dimensional translations and proper rotations; this group may be applicable to a $\mathrm{U}_{1}$ rotor moving in a plane.

\section{ACKNOWLEDGMENTS}

We thank Rafael N. Alexander, Benjamin L. Augenbraun, Stephen Bartlett, Juani Bermejo-Vega, Giacomo Bighin, Igor N. Cherepanov, David DeMille, Christa Flühmann, Steven M. Girvin, Alexey V. Gorshkov, Nick Hutzler, Joe Iverson, Arian Jadbabaie, Dominik Jüstel, Alexei Yu. Kitaev, Roman Korol, Roman Krems, Richard Kueng, Dietrich Leibfried, Mikhail Lemeshko, Angelo Lucia, Kang-Kuen Ni, Igor Pak, Hannes Pichler, Shruti Puri, Gil Refael, Grant Salton, Eugene Tang, and Jun Ye for useful suggestions and discussions. We gratefully acknowledge support from ARO-LPS (W911NF-18-10103), NSF (PHY-1733907), the Walter Burke Institute for Theoretical Physics, and the Division of Physics, Mathematics, and Astronomy (PMA) at Caltech. The Institute for Quantum Information and Matter is an NSF Physics Frontiers Center. Our figures were drawn using Mathematica 12.

\section{APPENDIX A: BUILDING CODES VIA MICROWAVE DRESSING}

As discussed in Sec. III, the most versatile approach to generating the approximate code words is to build a linear combination of angular-momentum eigenstates with the proper weights using an array of microwave couplings. For this preliminary scheme, we neglect hyperfine structure and mixing of momentum states with nuclear quadrupole moments [87]. As a concrete physical platform, we focus on molecules composed of bosonic isotopes of alkalineearth(-like) atoms [185] as well as ${ }^{12} \mathrm{C}$ and ${ }^{16} \mathrm{O}$, which have zero nuclear spin. A small electric field lifts the degeneracy of all $\left.\left|\begin{array}{c}\ell|m|,|n| \\ \ell\end{array}\right\rangle \leftrightarrow||_{\left|m^{\prime}\right|,\left|n^{\prime}\right|}^{\ell^{\prime}}\right\rangle$ transitions but does not significantly mix eigenstates. Similarly, a magnetic field can split the $\pm m$ and $\pm n$ degeneracies. Recall that the eigenenergies for a given $\ell$ are proportional to $\ell(\ell+1)$ [64]. Hence, any transition $\ell-\ell^{\prime}= \pm 1$ has a unique energy.

Since $B \sim 1 \mathrm{GHz}$ for most molecules, $\left.\left|{ }_{|m|,|n|}^{\ell}\right\rangle \leftrightarrow||_{\left|m^{\prime}\right|,\left|n^{\prime}\right|}^{\ell^{\prime}}\right\rangle$ transitions can conveniently be driven with microwave fields. Note also that the dipole matrix elements of such transitions are relatively large for polar molecules, often $\mu \approx 1$ atomic unit (a.u.). Therefore, multiphoton processes which are off resonant from intermediate states can still achieve sufficient couplings. Figure 6 shows the code words for $\mathrm{U}_{1}$ (a), $\mathrm{SO}_{3}$ (b), and $\mathrm{S}^{2}$ (c) in the angularmomentum basis, where the opacity qualitatively indicates the fractional population of the normalized state. Recall that, for $\mathrm{SO}_{3}$, we require only the $n=m$ states, so no explicit discussion of $n$ is necessary in any of these cases.

Consider, for example, ${ }^{1} \Sigma$-type molecules, such as bialkalis. Their orientations correspond to the state space $S^{2}$, and we consider realizing the code words (103) with $N=3$. We apply the damping function $e^{-(1 / 2) \Delta^{2} \hat{L}^{2}}$ from Sec. V C to normalize the code words. The parameter $\Delta>$ 0 depends on how many momentum states $\ell_{\max }$ we want to consider, while $\hat{L}^{2}$ is the total angular-momentum operator. The resulting logical zero approximate code word, up to normalization, is

$$
\left.|\tilde{0}\rangle \propto \sum_{\ell=0}^{\ell_{\max }} \sum_{|3 p| \leq \ell} e^{-(1 / 2) \Delta^{2} \ell(\ell+1)} Y_{3 p}^{\ell}\left(\frac{\pi}{2}, 0\right)||_{3 p}^{\ell}\right\rangle .
$$

We assume the molecule is initially in the rigid-rotor ground state $\left|\begin{array}{l}\ell \\ m\end{array}\right\rangle=\left|\begin{array}{l}0 \\ 0\end{array}\right\rangle$ and assemble the state (A1) using microwave tones. All couplings "cascade" down from $\left|\begin{array}{l}0 \\ 0\end{array}\right\rangle$ as shown in Fig. 6(c). Control over the frequency, power, polarization, and phase of a microwave tone can readily be achieved in the laboratory and is essential to build the state (A1). The microwave couplings are all adiabatic with respect to the timescales of molecular rotation, and we expect that continuous-wave microwave pulses are possible to build the code words as the steady state solution of the Hamiltonian with the microwave driving terms of the form

$$
\left.\Omega_{\ell m}^{\ell^{\prime} m^{\prime}} \exp \left[-i\left(\delta_{\ell m}^{\ell^{\prime} m^{\prime}} t+\phi_{\ell m}^{\ell^{\prime} m^{\prime}}\right)\right]\right|_{m^{\prime}} ^{\left.\ell^{\ell^{\prime}}\right\rangle\left\langle{ }_{m}^{\ell}\right|,},
$$

with Rabi frequency $\Omega_{\ell m}^{\ell^{\prime} m^{\prime}}$, detuning $\delta_{\ell m}^{\ell^{\prime} m^{\prime}}$, and phase $\phi_{\ell m}^{\ell^{\prime} m^{\prime}}$ in the rotating frame of the original rigid-rotor Hamiltonian $B \hat{L}^{2}$. 


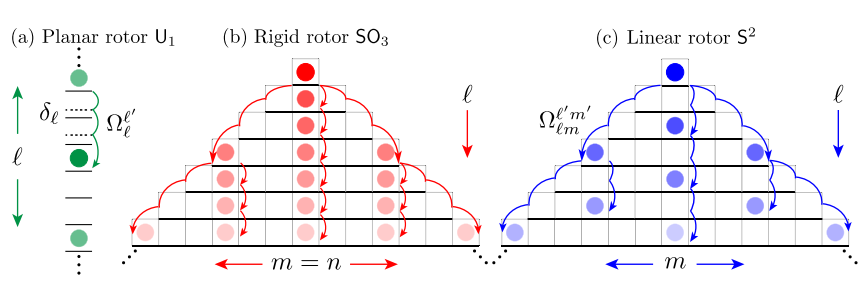

FIG. 6. Microwave dressing. Sketch of the microwave tones required to build the approximate code words out of angularmomentum eigenstates up to $\left|\ell_{\max }\right|=3$ for $\mathrm{U}_{1}$ (a) and $\ell_{\max }=6$ for $\mathrm{SO}_{3}$ (b) and $\mathrm{S}^{2}$ (c). We assume the molecule is initialized in $|\ell=0\rangle$ for $\mathrm{U}_{1},\left|\begin{array}{l}0 \\ 00\end{array}\right\rangle$ for $\mathrm{SO}_{3}$, and $\left|\begin{array}{l}0 \\ 0\end{array}\right\rangle$ for $\mathrm{S}^{2}$. An array of microwave tones is applied to construct the code words. Any transition allowed by dipole selection rules can be driven with a Rabi frequency $\Omega_{\ell m}^{\ell^{\prime} m^{\prime}}$, phase $\phi_{\ell m}^{\ell^{\prime} m^{\prime}}$, and detuning $\delta_{\ell m}^{\ell^{\prime} m^{\prime}}$. (For $\mathrm{SO}_{3}$, we utilize only the $n=m$ states, so no explicit mention of $n$ is necessary for those cases. For $\mathrm{U}_{1}$, there are only states with integer $\ell$.) Curved lines without arrows illustrate vastly offresonant coupling to a state for which the population is effectively zero, and the state can be adiabatically eliminated. Curved lines with arrows illustrate couplings with a finite population of the state. To prepare approximate code words in the subspaces shown, at most 12, 24, and 22 unique microwave tones are required for $\mathrm{U}_{1}, \mathrm{SO}_{3}$, and $\mathrm{S}^{2}$, respectively.

In the perturbative limit $\Omega / \delta \ll 1$, where we suppress the decorations on $\Omega$ and $\delta$ for clarity, the population $P_{\ell^{\prime} m^{\prime}}$ can be expressed as $P_{\ell^{\prime} m^{\prime}}=(\Omega / \delta)^{2} P_{\ell m}$, where $P_{\ell m}$ is the population in $\left|{ }_{m}^{\ell}\right\rangle$. We ignore the Stark shift of ||$\left._{m^{\prime}}^{\ell^{\prime}}\right\rangle$, given by $\delta^{S}=\Omega^{2} / \delta$, since $\delta^{S} \ll \delta$. Applying the same analysis to multiphoton processes, now consider the three-photon pulses $\left.\left|\begin{array}{l}\ell_{0} \\ m_{0}\end{array}\right\rangle \rightarrow||_{m_{1}}^{\ell_{1}}\right\rangle \rightarrow\left|\begin{array}{l}\ell_{2} \\ m_{2}\end{array}\right\rangle \rightarrow\left|\begin{array}{l}\ell_{3} \\ m_{3}\end{array}\right\rangle$. Assuming vastly offresonant drives for the intermediate states, we can adiabatically eliminate said states. The population in $\left|\begin{array}{l}\ell_{3} \\ m_{3}\end{array}\right\rangle$ is given by

$$
P_{\ell_{3}, m_{3}}=\left(\frac{\Omega_{\ell_{0} m_{0}}^{\ell_{1} m_{1}} \Omega_{\ell_{1} m_{1}}^{\ell_{2} m_{2}} \Omega_{\ell_{2} m_{2}}^{\ell_{3} m_{3}}}{\delta_{\ell_{0} m_{0}}^{\ell_{1} m_{1}} \delta_{\ell_{1} m_{1}}^{\ell_{2} m_{2}} \delta_{\ell_{2} m_{2}}^{\ell_{3} m_{3}}}\right)^{2} P_{\ell_{0} m_{0}} .
$$

Thus, $\left|l_{m_{3}}^{l_{3}}\right\rangle$ can effectively be attained from $\left|l_{m_{0}}^{l_{0}}\right\rangle$ using the above combination of tones.

The above scheme can be used to transfer population in each momentum state present in the approximate state (A1) to neighboring states. That way, the population in $\left|\begin{array}{l}0 \\ 0\end{array}\right\rangle$ cascades down the angular-momentum pyramid. The required tones are shown in Fig. 6. Curved lines with arrows illustrate couplings with a finite population of the state. For the $\mathrm{S}^{2}$ state (A1) with $\ell_{\max }=6$, we require 22 unique microwave tones. Similar schemes for $\mathrm{U}_{1}$ and $\mathrm{SO}_{3}$, shown in Figs. 6(a) and 6(b), require 12 and 24 tones, respectively.

The above is just a sketch. A detailed analysis based on, e.g., exact diagonalization of the coupling Hamiltonian or a master equation is required to choose the frequency, power, polarization, and phase of each microwave tone (A2). One must be aware of the formation of dark states as well as potentially dynamic evolution of the populations. Advanced modeling is particularly important outside the perturbative limit when $\Omega_{\ell m}^{\ell^{\prime} m^{\prime}} / \delta_{\ell m}^{\ell^{\prime} m^{\prime}} \sim 1$, for which population dynamics must be addressed. Time-dependent pulses can also be employed for which the power and phase can be adjusted on timescales comparable to the Rabi frequencies (but still slow compared to rotational timescales). Such an analysis is outside the scope of this work.

An electric field may be helpful for building the code words and could potentially reduce the number of required microwave tones. In our notation, an electric field is represented by operator $\cos \hat{\phi}$ for $\mathrm{U}_{1}, \hat{D}_{00}^{1}$ for $\mathrm{SO}_{3}$, and $\hat{Y}_{0}^{1}$ for $\mathrm{S}^{2}$. For the latter two spaces, a large electric field mixes $\ell$ states with the same $m$ [see Eq. (60)]. This point is particularly salient in the case of $\mathrm{SO}_{3}$, where couplings down the columns for $|m|=0,3,6$ are required. A large electric field naturally creates these couplings down each column in Fig. 6(b).

\section{APPENDIX B: VORONOI CELLS}

We determine the Voronoi-Dirichlet cells of the quotient spaces $\mathrm{SO}_{3} / \mathrm{H}$ from Table I. This determination is strictly an adaptation of the work of Postnikov [92] (see also [95]).

Let $\mathrm{X}$ be a metric space with distance function $\mathrm{d}$ and distinguished origin point $x_{0}$. Let $\mathrm{H}$ be a discrete group whose elements $R \in \mathrm{H}$ map points $x \in \mathrm{X}$ as $x \rightarrow R x$. This group maps the origin to the orbit $\left\{R x_{0}\right\}_{R \in \mathrm{H}}$. Each such point $R x_{0}$ has its own Voronoi cell-a region consisting of points that are closer (or as close) to $R x_{0}$ than to $R^{\prime} x_{0}$ for any $R^{\prime} \neq R$. When $R$ is the identity, we call the corresponding cell the fundamental Voronoi cell,

$$
\mathrm{F}_{\mathrm{X} / \mathrm{H}}=\left\{x \in \mathrm{X} \mid \quad \forall R \in \mathrm{H}, \mathrm{d}\left(x, x_{0}\right) \leq \mathrm{d}\left(x, R x_{0}\right)\right\} .
$$

When the space is a group $(\mathrm{X}=\mathrm{G}), x_{0}$ is the identity, and the above is the Voronoi cell of the identity.

To properly account for distances in $\mathrm{SO}_{3}$, we recall that it is equivalent to the 3 -sphere $\mathrm{S}^{3}=\mathrm{SU}_{2}$ with opposite points identified, $\mathrm{SO}_{3}=\mathrm{S}^{3} / \mathrm{Z}_{2}$ [153]. The 3-sphere can be parameterized by either a four-dimensional unit vector or by a quaternion. Just like a semicircle parameterizes a circle with opposite points identified, hemispherical quanternions

$$
\eta(\omega, \mathbf{v})=\left(\cos \frac{\omega}{2}, \mathbf{v} \sin \frac{\omega}{2}\right)
$$

are an equivalent way to parameterize $R=(\omega, \mathbf{v}) \in \mathrm{SO}_{3}$. The distance function $d$ we use is the dot product of the above 4-vectors.

The Voronoi cell of a point $p$ is bounded by the mediatrices of $p$ and all $q$ that are in the orbit of $p$ under $\mathrm{H}$. A mediatrix of points $p$ and $q$ is the set of all points that lie the same distance from $p$ and $q$. For a 2D square lattice, 
the boundary of $\mathrm{F}_{\mathrm{R}^{\times 2} / \mathrm{Z}^{\times 2}}$ consists of segments that bisect lines that connect the center of the cell with centers of neighboring cells. The boundaries of $\mathrm{F}_{\mathrm{SO}_{3} / \mathrm{H}}$ are hyperplanes that go through the midpoint $(\Omega / 2, \mathbf{w})$ of the geodesic connecting the center with each group element $(\Omega, \mathbf{w}) \in \mathrm{H}$. Points $\eta$ on such hyperplanes satisfy

$$
\dot{\eta}(\Omega / 2, \mathbf{w}) \cdot \eta(\omega, \mathbf{v})=0,
$$

where $\dot{\eta} \equiv \partial \eta / \partial \omega$. Solving for $\omega$ yields

$$
\omega=\left|2 \cot ^{-1}\left(\mathbf{v} \cdot \mathbf{w} \cot \frac{\Omega}{4}\right)\right| .
$$

We use this to plot the various manifolds in Figs. 2-4. Letting $\mathrm{H}$ be a $\mathbf{z}$-axis rotation subgroup yields Eq. (53).

\section{APPENDIX C: NORMALIZABLE CODE WORDS}

\section{Observables}

For the calculation of $\bar{\ell}$ (77), in the $\Delta \rightarrow 0$ limit

$$
\left\langle\tilde{r}\left|\hat{L}^{2}\right| \tilde{r}\right\rangle=\frac{\left\langle\bar{r}\left|\hat{L}^{2} e^{-\Delta^{2} \hat{L}^{2}}\right| \bar{r}\right\rangle}{\left\langle\bar{r}\left|e^{-\Delta^{2} \hat{L}^{2}}\right| \bar{r}\right\rangle} \sim \frac{\left\langle I\left|\hat{L}^{2} e^{-\Delta^{2} \hat{L}^{2}}\right| I\right\rangle}{\left\langle I\left|e^{-\Delta^{2} \hat{L}^{2}}\right| I\right\rangle},
$$

where $I$ is the identity rotation. To obtain the above, recall that each $|\bar{r}\rangle$ is a superposition of position states $\left|R_{(2 \pi / N) h+(\pi / N) r, \mathbf{z}}\right\rangle$ for $h \in \mathrm{Z}_{N}$. The state $e^{-\Delta^{2} \hat{L}^{2}}\left|R_{(2 \pi / N) h+(\pi / N) r, \mathbf{z}}\right\rangle$ can be thought of as a Gaussian distribution of orientations centered at $\left|R_{(2 \pi / N) h+(\pi / N) r, \mathbf{z}}\right\rangle$, overlapping with other states for different $h$. However, since the overlap is exponentially suppressed with $h$, we ignore such contributions. We then use the fact that all rotations commute with $\hat{L}^{2}$, allowing us to remove $h, r$ dependence:

$$
\left\langle R_{\omega, \mathbf{v}}\left|f\left(\hat{L}^{2}\right)\right| R_{\omega, \mathbf{v}}\right\rangle=\left\langle I\left|f\left(\hat{L}^{2}\right)\right| I\right\rangle
$$

for any $(\omega, \mathbf{v})$ and function $f\left(\hat{L}^{2}\right)$.

Having used $\mathrm{SO}_{3}$ symmetry to remove dependence of $(\omega, \mathbf{v})$, we now express the identity state in the momentum basis, yielding

$$
\left\langle I\left|f\left(\hat{L}^{2}\right)\right| I\right\rangle=\frac{1}{8 \pi^{2}} \sum_{\ell \geq 0}(2 \ell+1)^{2} f[\ell(\ell+1)] .
$$

The identity state is supported only on momentum states $|\ell m\rangle$ with amplitude $(2 \ell+1) / 8 \pi^{2}$, and we perform the sum over $m$ to obtain the extra $2 \ell+1$ factor. The function $f\left(\hat{L}^{2}\right)$ becomes as such due to Eq. (75). We then rearrange the above sum to obtain a sum over integers, which can then be approximated using Poisson summation (D22) for the relevant $f$ :

$$
\begin{aligned}
\left\langle I\left|f\left(\hat{L}^{2}\right)\right| I\right\rangle & =\frac{1}{16 \pi^{2}} \sum_{\ell \in Z}(2 \ell+1)^{2} f[\ell(\ell+1)] \\
& \sim \frac{1}{16 \pi^{2}} \int_{\mathrm{R}} d x(2 x+1)^{2} f[x(x+1)] .
\end{aligned}
$$

Plugging in explicit forms for $f$ yields Eq. (77).

\section{Leakage error}

To evaluate $P_{\text {leak }}$, we first start with its complement $P_{\mathrm{ok}}=1-P_{\text {leak }}$, the projection of $|\tilde{0}\rangle$ onto its own Voronoi cells [for $N=3$, those in the left in Fig. 3(c)]:

$$
P_{\mathrm{ok}}=\sum_{h \in \mathrm{Z}_{N}} \int_{\mathrm{F}_{\mathrm{SO}_{3} / Z_{2 N}}} d S_{\omega, \mathbf{v}}\left|\left\langle S_{\omega, \mathbf{v}} R_{(2 \pi / N) h, \mathbf{z}} \mid \tilde{0}\right\rangle\right|^{2} .
$$

Recall that $|\tilde{0}\rangle$ is a superposition of smeared group elements $\left|R_{(2 \pi / N) k, \mathbf{z}}\right\rangle$ for $k \in \mathrm{Z}_{N}$. Inserting this expansion for $|\tilde{0}\rangle$, we estimate $P_{\mathrm{ok}}$ in the $\Delta \rightarrow 0$ limit by ignoring contributions from elements leaking outside of their own Voronoi cells:

$$
\begin{aligned}
P_{\mathrm{ok}} \sim & \sum_{h \in \mathrm{Z}_{N}} \int_{\mathrm{F}_{\mathrm{SO}_{3} / Z_{2 N}}} d S_{\omega, \mathbf{v}} \\
& \times \frac{\left|\left\langle S_{\omega, \mathbf{v}} R_{(2 \pi / N) h, \mathbf{z}}\left|e^{-(1 / 2) \Delta^{2} \hat{L}^{2}}\right| R_{(2 \pi / N) h, \mathbf{z}}\right\rangle\right|^{2}}{N\left\langle\overline{0}\left|e^{-\Delta^{2} \hat{L}^{2}}\right| \overline{0}\right\rangle} .
\end{aligned}
$$

Inserting the asymptotic expression for $\left\langle\overline{0}\left|e^{-\Delta^{2} \hat{L}^{2}}\right| \overline{0}\right\rangle$ and using invariance under rotations $(\mathrm{C} 2)$ brings us to

$P_{\mathrm{ok}} \sim \frac{8(\sqrt{\pi} \Delta)^{3}}{e^{\Delta^{2} / 4}} \int_{\mathrm{F}_{\mathrm{SO}_{3} / Z_{2 N}}} d S_{\omega, \mathbf{v}}\left|\left\langle S_{\omega, \mathbf{v}}\left|e^{-(1 / 2) \Delta^{2} \hat{L}^{2}}\right| I\right\rangle\right|^{2}$.

Now we get rid of the $\mathbf{v}$ dependence of the absolute value. Recall that rotations conjugate each other as

$$
R_{\Omega, \mathrm{w}} R_{\omega, \mathbf{v}} R_{\Omega, \mathrm{w}}^{\dagger}=R_{\omega, R_{\Omega, \mathrm{w}} \mathbf{v}}
$$

For each $\mathbf{v}$, we pick $(\Omega, \mathbf{w})$ such that $R_{\Omega, \mathbf{w}} \mathbf{v}=\mathbf{z}$. We then "create" that rotation inside the ket and commute it through to the bra:

$$
\begin{aligned}
\left\langle S_{\omega, \mathbf{v}}\left|f\left(\hat{L}^{2}\right)\right| I\right\rangle & =\left\langle S_{\omega, \mathbf{v}}\left|f\left(\hat{L}^{2}\right)\right| R_{\Omega, \mathbf{w}}^{\dagger} R_{\Omega, \mathbf{w}}\right\rangle \\
& =\left\langle R_{\Omega, \mathbf{w}} S_{\omega, \mathbf{v}} R_{\Omega, \mathbf{w}}^{\dagger}\left|f\left(\hat{L}^{2}\right)\right| I\right\rangle \\
& =\left\langle S_{\omega, \mathbf{z}}\left|f\left(\hat{L}^{2}\right)\right| I\right\rangle .
\end{aligned}
$$

We further estimate this matrix element via the same procedure as that for the average angular momentum: 


$$
\begin{aligned}
\left\langle S_{\omega, \mathbf{Z}}\left|e^{-(1 / 2) \Delta^{2} \hat{L}^{2}}\right| I\right\rangle & \sim \int_{\mathrm{R}} d x \frac{2 x+1}{e^{\left(\Delta^{2} / 2\right) x(x+1)}} \frac{\sin [(2 x+1) \omega / 2]}{16 \pi^{2} \sin (\omega / 2)} \\
& =\frac{\sqrt{2}}{8} \frac{\omega e^{\left(\Delta^{2} / 8\right)-\left(\omega^{2} / 2 \Delta^{2}\right)}}{(\sqrt{\pi} \Delta)^{3} \sin (\omega / 2)} . \quad(\mathrm{C} 10)
\end{aligned}
$$

Plugging all of the above into $P_{\text {leak }}=1-P_{\text {ok }}$ yields

$$
P_{\text {leak }} \sim 1-\frac{1}{4(\sqrt{\pi} \Delta)^{3}} \int_{\mathrm{F}_{\mathrm{SO}_{3} / \mathrm{Z}_{2 N}}} d S_{\omega, \mathbf{v}} \frac{\omega^{2} e^{-\omega^{2} / \Delta^{2}}}{\sin ^{2}(\omega / 2)} .
$$

The integration measure (see Sec. 4.5.4 in Ref. [109]) is

$$
d S_{\omega, \mathbf{v}}=4 \sin \Theta \sin ^{2}(\omega / 2) d \Theta d \Phi d \omega,
$$

and, for $\mathrm{F}_{\mathrm{SO}_{3} / \mathrm{Z}_{2 N}}$, the integration of $(\Theta, \Phi)=\mathbf{v}$ is over $S^{2}$ and $\omega \in\left[0, \omega_{\max }(\Theta)\right]$ [see Eq. (53)]. To absorb the "1-" part, we integrate over the complementary region, for which $\omega \in\left[\omega_{\max }(\Theta), \pi\right]$. Trivially integrating over the azimuthal angle and simplifying yields

$$
P_{\text {leak }} \sim \frac{2}{\sqrt{\pi} \Delta^{3}} \int_{0}^{\pi} \sin \Theta \beta \Theta \int_{\omega_{\max }(\Theta)}^{\pi} d \omega \omega^{2} e^{-\omega^{2} / \Delta^{2}} .
$$

To simplify the integral further, we first use invariance under $\Theta \rightarrow \pi-\Theta$ to write

$$
P_{\text {leak }} \sim \frac{4}{\sqrt{\pi} \Delta^{3}} \int_{0}^{\pi / 2} \sin \Theta d \Theta \int_{\omega_{\star}}^{\pi} d \omega \omega^{2} e^{-\omega^{2} / \Delta^{2}},
$$

where, due to the new integration domain, we can remove the absolute value in $\omega_{\max }$ and define $\omega_{\star} \equiv 2 \cot ^{-1}[\cos \Theta \cot (\pi / 4 N)]$. Now we apply Laplace's method [186]: In the $\Delta \rightarrow 0$ limit, the leading-order contribution to the $\omega$ integral is around $\omega=\omega_{\star}$, since the exponential $\omega^{2} / \Delta^{2}$ is minimized there. Thus, we can increase the upper $\omega$ bound to infinity without losing accuracy and perform the resulting Gaussian-type integral. Plugging the result into the remaining integral yields

$$
P_{\text {leak }} \sim \frac{2}{\sqrt{\pi} \Delta} \int_{0}^{\pi / 2} d \Theta \omega_{\star} \sin \Theta e^{-\omega_{\star}^{2} / \Delta^{2}} .
$$

Now the dominant contribution is around $\Theta=0$. Expressing $\omega_{\star}$ in terms of $\Theta$, we expand both $\omega_{\star} \sin \Theta$ and the exponential around zero:

$$
\begin{gathered}
\omega_{\star}^{2} \approx\left(\frac{\pi}{2 N}\right)^{2}+\frac{\pi}{2 N} \sin \left(\frac{\pi}{2 N}\right) \Theta^{2}, \\
\omega_{\star} \sin \Theta \approx \frac{\pi}{2 N} \Theta .
\end{gathered}
$$

Plugging this result in, extending the upper bound to infinity, evaluating the resulting Gaussian-type integral, and simplifying yields the result (78).

\section{Momentum-kick distortion}

A way of understanding why detection of $\ell<N$ momentum kicks implies correction of $\ell<N / 2$ kicks stems from the fact that products of $D_{m n}^{\ell}(R) D_{m^{\prime} n^{\prime}}^{\ell^{\prime}}(R)$ can be expanded in terms of a sum of single $D$ matrices (see Sec. 4.6.1 in Ref. [109]). Upgrading this expansion to operators and using selection rules (60) yields

$$
\hat{D}_{m n}^{\ell} \hat{D}_{m^{\prime} n^{\prime}}^{\ell^{\prime}}=\sum_{L=\left|\ell^{\prime}-\ell\right|}^{\ell^{\prime}+\ell} C_{\ell m \ell^{\prime} m^{\prime}}^{L, m^{\prime}+m} C_{\ell n \ell^{\prime} n^{\prime}}^{L, n^{\prime}+n} \hat{D}_{m^{\prime}+m, n^{\prime}+n}^{L},
$$

where the Clebsch-Gordan coefficients $C_{\ell m \ell^{\prime} m^{\prime}}^{L, m^{\prime}+m}=0$ when $\left|m^{\prime}+m\right|,\left|n^{\prime}+n\right|>L$. Per the Knill-Laflamme conditions, in order to correct against kicks by angular momentum $\ell<N / 2$, we need to detect products of kicks $\left(\hat{D}_{m n}^{\ell}\right)^{\dagger} \hat{D}_{m^{\prime} n^{\prime}}^{\ell^{\prime}}$ with $\ell, \ell^{\prime}<N / 2$. Using the above equation and

$$
\left(\hat{D}_{m n}^{\ell}\right)^{\dagger}=(-1)^{m+n} \hat{D}_{-m,-n}^{\ell}
$$

(see Sec. 4.5 in Ref. [109]), such products can be expanded in terms of single kicks with momentum $<N$. Detection of such single kicks thus implies correction of kicks with $\ell<N / 2$.

For the normalizable code words, the problem comes from violations of the Knill-Laflamme conditions stemming from $\hat{D}_{00}^{\ell}$, namely, $\left\langle\tilde{0}\left|\hat{D}_{00}^{\ell}\right| \tilde{1}\right\rangle \neq 0$. Using Eq. (C16), this problem translates to not being able to perfectly resolve kicks $\hat{D}_{m m}^{\ell}$ with fixed $m$ but different $\ell$, as the distortion caused by such kicks depends on $\ell$. Working in the $\Delta \rightarrow 0$ limit, plugging in the approximate code word normalization, using Table IV G, and using selection rules (60),

$$
\begin{aligned}
\left\langle\tilde{0}\left|\hat{D}_{00}^{\ell}\right| \tilde{1}\right\rangle \sim & \frac{\Delta^{3} N}{e^{\Delta^{2} / 4} \sqrt{\pi}} \sum_{\ell^{\prime} \geq 0}\left(2 \ell^{\prime}+1\right) e^{-\left(\Delta^{2} / 2\right) \ell^{\prime}\left(\ell^{\prime}+1\right)} \\
& \times \sum_{\left|\ell^{\prime}-\ell\right| \leq L \leq \ell^{\prime}+\ell} e^{-\left(\Delta^{2} / 2\right) L(L+1)} \\
& \times \sum_{|P N| \leq L}(-1)^{P}\left(C_{\ell, 0, \ell^{\prime}, P N}^{L, P N}\right)^{2} .
\end{aligned}
$$

Numerically, this sum is exponentially suppressed with $1 / \Delta^{2}$ for all $\ell<N$ that we test. Below, we estimate its asymptotic behavior, showing that its dependence on $\Delta$ is surprisingly similar to $P_{\text {leak }}(78)$.

As $\Delta \rightarrow 0$, ever-increasing values of $L, \ell^{\prime}$ contribute to the sums (C18). For $\ell \ll L, \ell^{\prime}$, the Clebsch-Gordan coefficients $C_{\ell, 0, \ell^{\prime}, P N}^{L, N}$ approach their semiclassical limita particular $D$-matrix element (see Sec. 8.9.1 in Ref. [109]). Since $D$ matrices are unitary, this element is bounded by one. Setting all $C$ 's to one and evaluating the innermost sum over $P$ yields 
TABLE V. Summary of relations for coset spaces $\mathcal{L}^{2}(\mathrm{G} / \mathrm{H})$, treated as subspaces of group spaces $\mathcal{L}^{2}(\mathrm{G})$; see Appendix D. The $C_{\ell m \ell^{\prime} m^{\prime}}^{L M}$ are Clebsch-Gordan coefficients [109,153]. The operator $\hat{X}_{h}$ is $\vec{X}_{h}$ projected onto the quotient space.

\begin{tabular}{|c|c|c|}
\hline & Coset space $\mathcal{L}^{2}(\mathrm{G} / \mathrm{H}) \subset \mathcal{L}^{2}(\mathrm{G})$ & Linear rotor $\mathcal{L}^{2}\left(\mathrm{~S}^{2}\right) \subset \mathcal{L}^{2}\left(\mathrm{SO}_{3}\right)$ \\
\hline A. Phase space & $\left(a,{ }_{m n}^{\ell}\right) \in \mathrm{G} / \mathrm{H} \times \widehat{\mathrm{G} / \mathrm{H}}$ & $(\mathbf{v}, l, m) \in S^{2} \times \widehat{S^{2}}$ \\
\hline B. Conjugate bases & $\begin{aligned}|a \mathrm{H}\rangle & \left.=\left.\sum_{\ell m n \in \mathrm{G} / \mathrm{H}} \sqrt{\left(d_{\ell} /|\mathrm{G} / \mathrm{H}|\right)} Z_{m n}^{\ell \star}(a \mathrm{H})\right|_{m n} ^{\ell}\right\rangle \\
\left|{ }_{m n}^{\ell}\right\rangle & =\sum_{a \in \mathrm{G} / \mathrm{H}} \sqrt{\left(d_{\ell} /|\mathrm{G} / \mathrm{H}|\right)} Z_{m n}^{\ell}(a \mathrm{H})|a \mathrm{H}\rangle\end{aligned}$ & $\begin{array}{c}\left.|\mathbf{v}\rangle=\left.\sum_{\ell \geq 0} \sum_{|m| \leq \ell} Y_{m}^{\ell \star}(\mathbf{v})\right|_{m} ^{\ell}\right\rangle \\
\left|{ }_{m}^{\ell}\right\rangle=\int_{\mathrm{S}^{2}} d \mathbf{v} Y_{m}^{\ell}(\mathbf{v})|\mathbf{v}\rangle\end{array}$ \\
\hline C. Overlap & $\left\langle a \mathrm{H} \mid{ }_{m n}^{\ell}\right\rangle=\sqrt{\left(d_{\ell} /|\mathrm{G} / \mathrm{H}|\right)} Z_{m n}^{\ell}(a \mathrm{H})$ & $\left\langle\left.\mathbf{v}\right|_{m} ^{\ell}\right\rangle=Y_{m}^{\ell}(\mathbf{v})$ \\
\hline D. Resolution & $\begin{array}{c}\sum_{a \in \mathrm{G} / \mathrm{H}}|a \mathrm{H}\rangle\langle a \mathrm{H}| \\
=\sum_{\ell m n \in \widehat{\mathrm{G} / \mathrm{H}}}\left|{ }_{m n}^{\ell}\right\rangle\left\langle\left\langle_{m n}^{\ell}\right|=1_{\mathrm{G} / \mathrm{H}}\right.\end{array}$ & $\int_{\mathrm{S}^{2}} d \mathbf{v}|\mathbf{v}\rangle\left\langle\mathbf{v}\left|=\sum_{\ell \geq 0} \sum_{|m| \leq \ell}\right|_{m}^{\ell}\right\rangle\left\langle\left\langle_{m}^{\ell}\right|=1_{\mathrm{S}^{2}}\right.$ \\
\hline E. Orthocompleteness & $\begin{array}{c}\sum_{a \in \mathrm{G} / \mathrm{H}} Z_{m n}^{\ell \star}(a \mathrm{H}) Z_{m^{\prime} n^{\prime}}^{\ell^{\prime}}(a \mathrm{H}) \\
=\left(|\mathrm{G} / \mathrm{H}| / d_{\ell}\right) \delta_{\ell \ell^{\prime}} \delta_{m m^{\prime}} \delta_{n n^{\prime}} \\
\sum_{\ell m n \in \widehat{\mathrm{G} / \mathrm{H}}}\left(d_{\ell} /|\mathrm{G} / \mathrm{H}|\right) Z_{m n}^{\ell \star}(a \mathrm{H}) Z_{m n}^{\ell}\left(a^{\prime} \mathrm{H}\right)=\delta_{a a^{\prime}}^{\mathrm{G} / \mathrm{H}}\end{array}$ & $\begin{array}{c}\int_{\mathrm{S}^{2}} d \mathbf{v} Y_{m}^{\ell \star}(\mathbf{v}) Y_{m^{\prime}}^{\ell^{\prime}}(\mathbf{v})=\delta_{\ell \ell^{\prime}} \delta_{m m^{\prime}} \\
\sum_{\ell \geq 0} \sum_{|m| \leq \ell} Y_{m}^{\ell \star}(\mathbf{v}) Y_{m}^{\ell}\left(\mathbf{v}^{\prime}\right)=\delta_{\mathbf{v v}^{\prime}}^{\mathrm{S}^{2}}\end{array}$ \\
\hline F. Position shifts & $\begin{array}{c}\hat{X}_{h}|a \mathrm{H}\rangle=|h a \mathrm{H}\rangle \\
\left.\hat{X}_{h}\left|{ }_{m n}^{\ell}\right\rangle=\left.\sum_{p} Z_{p m}^{\ell \star}(h)\right|_{p n} ^{\ell}\right\rangle\end{array}$ & $\begin{array}{c}\hat{X}_{R}|\mathbf{v}\rangle=|R \mathbf{v}\rangle \\
\left.\hat{X}_{R}\left|{ }_{m}^{\ell}\right\rangle=\left.\sum_{|p| \leq \ell} D_{p m}^{\ell \star}(R)\right|_{p} ^{\ell}\right\rangle\end{array}$ \\
\hline G. Phase shifts & $\begin{array}{c}\hat{Z}_{m n}^{\ell}(\mathrm{H})|a \mathrm{H}\rangle=Z_{m n}^{\ell}(a \mathrm{H})|a \mathrm{H}\rangle \\
\left.\left.\hat{Z}_{m n}^{\ell}(\mathrm{H})\right|_{m^{\prime} n^{\prime} n^{\prime}} ^{\ell}=\left.\sum_{L M N \in \widehat{G} / \mathrm{H}} c_{\ell m n n \ell^{\prime} m^{\prime} n^{\prime}}^{L M N}\right|_{M N} ^{L}\right\rangle \\
c_{\ell m n, \ell^{\prime} m^{\prime} n^{\prime}}^{L M}=\left(\sqrt{d_{\ell^{\prime}} d_{L}} /|\mathrm{G} / \mathrm{H}|\right) \\
\sum_{a \in \mathrm{G} / \mathrm{H}} Z_{M N}^{L \star}(a \mathrm{H}) Z_{m^{\prime} n^{\prime}}^{\ell^{\prime}}(a \mathrm{H}) Z_{m n}^{\ell}(a \mathrm{H})\end{array}$ & $\begin{array}{c}\hat{Y}_{m}^{\ell}|\mathbf{v}\rangle=Y_{m}^{\ell}(\mathbf{v})|\mathbf{v}\rangle \\
\left.\left.\hat{Y}_{m}^{\ell}||_{m^{\prime}}^{\ell^{\prime}}\right\rangle=\left.\sum_{L \geq 0} \sum_{|M| \leq L} c_{\ell m, \ell^{\prime} m^{\prime}}^{L M}\right|_{M} ^{L}\right\rangle \\
c_{\ell m, \ell^{\prime} m^{\prime}}^{L M}=\sqrt{\left[(2 l+1)\left(2 l^{\prime}+1\right) / 4 \pi(2 L+1)\right]} \\
C_{\ell m \ell^{\prime} m^{\prime}}^{L M} C_{\ell 0 \ell^{\prime} 0^{\prime}}^{L 0}\end{array}$ \\
\hline H. Weyl relation & $\hat{X}_{h} \hat{Z}_{m n}^{\ell}(\mathrm{H}) \hat{X}_{h}^{\dagger}=\sum_{p} Z_{p m}^{\ell \star}(h) \hat{Z}_{p n}^{\ell}(\mathrm{H})$ & $\hat{X}_{R} \hat{Y}_{m}^{\ell} \hat{X}_{R}^{\dagger}=\sum_{|p| \leq \ell} D_{p m}^{\ell \star}(R) \hat{Y}_{p}^{\ell}$ \\
\hline
\end{tabular}

$$
\begin{aligned}
\left\langle\tilde{0}\left|\hat{D}_{00}^{\ell}\right| \tilde{1}\right\rangle \approx & \frac{\Delta^{3} N}{e^{\Delta^{2} / 4} \sqrt{\pi}} \sum_{\ell^{\prime} \geq 0}\left(2 \ell^{\prime}+1\right) e^{-\left(\Delta^{2} / 2\right) \ell^{\prime}\left(\ell^{\prime}+1\right)} \\
& \times \sum_{\left|\ell^{\prime}-\ell\right| \leq L \leq \ell^{\prime}+\ell} e^{-\left(\Delta^{2} / 2\right) L(L+1)}(-1)^{\lfloor L / N\rfloor} .
\end{aligned}
$$

For all $\ell^{\prime}>\ell$, there are $2 \ell+1$ different values of $L$. For each value, we approximate the resulting sum over $\ell^{\prime}$ by the sum associated with $L=\ell^{\prime}$. This approximation yields

$$
\begin{aligned}
\left\langle\tilde{0}\left|\hat{D}_{00}^{\ell}\right| \tilde{1}\right\rangle \approx & \frac{(2 \ell+1) \Delta^{3} N}{e^{\Delta^{2} / 4} \sqrt{\pi}} \\
& \times \sum_{\ell^{\prime} \geq 0}\left(2 \ell^{\prime}+1\right) e^{-\Delta^{2} \ell^{\prime}\left(\ell^{\prime}+1\right)}(-1)^{\left\lfloor\ell^{\prime} / N\right\rfloor} .
\end{aligned}
$$

The sign of the summand oscillates with $\ell^{\prime}$, with periodicity $N$. Numerically, we observe that this expression scales as the quoted estimate (79).

\section{APPENDIX D: COSET SPACES $\mathcal{L}^{2}(\mathbf{G} / \mathrm{H})$}

In close analogy to group spaces $\mathrm{G}$, here we construct Table V—an analog to Table IV for coset spaces $\mathrm{G} / \mathrm{H}$ with $\mathrm{H} \subseteq \mathrm{G}$. The key idea is to treat these spaces as subspaces of the group space $G$, which allows us to develop shift operators and orthogonality relations. This treatment is intended for molecular state spaces $\mathrm{SO}_{3} / \mathrm{H}$, but it also provides a framework for qudit-type spaces $\mathrm{G} / \mathrm{H}$ for finite
$\mathrm{G}$ as well as symmetric spaces for $\mathrm{G}$ a Lie group. However, as with Sec. VII, we consider finite G to better flesh out the key intuition.

Mathematically, our result is a "coordinates statement" of the Peter-Weyl theorem for homogeneous spaces [see Corollary 9.14 in Ref. [187], Ref. [188], Eq. (116) in Ref. [179], and Sec. II. 3.9 in Ref. [189] ]. The ability to make such a statement stems from a particular choice of the "coordinates," namely, bases for the irreps of G that are block diagonal when restricted to $\mathrm{H}$.

Recalling that a space $\mathrm{G}$ consists of states $\{|g\rangle, g \in \mathrm{G}\}$, the defining position states of the space $\mathrm{G} / \mathrm{H}$ are equal superpositions of elements of cosets of $\mathrm{H}$ in $\mathrm{G}$ :

$$
|a \mathrm{H}\rangle \equiv \frac{1}{\sqrt{|\mathrm{H}|}} \sum_{h \in \mathrm{H}}|a h\rangle,
$$

where $a$ is any element of the coset $a \mathrm{H}$. In effect, projecting into the subspace spanned by the above states is equivalent to performing the quotient map on the level of the group. Some applications require unique choices of coset representatives, so we pick $a \in \mathrm{F}_{\mathrm{G} / \mathrm{H}}$ - the Voronoi cell of the identity (see Appendix B). We abuse notation and use $\mathrm{G} / \mathrm{H}$ and $\mathrm{F}_{\mathrm{G} / \mathrm{H}}$ interchangeably throughout the paper.

Expressing these in terms of the momentum states of $\mathrm{G}$ from Table IV B, switching sums, and using definition (128) yields a generalization of Eq. (127) to all G/H: 


$$
\left.|a \mathrm{H}\rangle=\left.\sum_{\ell m n \in \hat{\mathrm{G}}} \sqrt{\frac{d_{\ell}}{|\mathrm{G} / \mathrm{H}|}} Z_{m n}^{\ell \star}(a \mathrm{H})\right|_{m n} ^{\ell}\right\rangle .
$$

We observe that this equation is zero unless $Z^{\ell}(\mathrm{H}) \neq 0$. The participating $\ell$ thus form the reciprocal space [127]

$$
\mathrm{H}^{\perp}=\left\{\ell \in \hat{\mathrm{G}}, Z^{\ell}(\mathrm{H}) \neq 0\right\},
$$

a notion that generalizes the dual or reciprocal lattice for $\mathrm{H}=\mathrm{Z}^{\times d}[190]$ and the dual code for CSS codes $\mathrm{H}=\mathrm{Z}_{2}^{\times n}$ (see Lemma 7.1 in Ref. [141]).

The reciprocal space depends significantly on $G$ and $H$. For example, the $\mathrm{Z}_{3} \subset \mathrm{Z}_{6} \subset \mathrm{U}_{1}$ code words (45) contain every third $\ell, \mathrm{Z}_{3}^{\perp}=\left\{\ell \in \mathrm{Z}, e^{i(2 \pi / 3) \ell}=1\right\}$. For the $\mathrm{T} \subset$ $\mathrm{O} \subset \mathrm{SO}_{3}$ code from Sec. VE, $\ell \in\{1,2,5\}$ do not participate. For $\mathrm{Z}_{3} \subset \mathrm{Z}_{6} \subset \mathrm{SO}_{3}$ (48), all $\ell \in \widehat{\mathrm{SO}_{3}}$ participate, but the internal indices $m$ and $n$ are restricted.

While the surviving $\ell$ are established by $\mathrm{H}^{\perp}$, surviving $m$ and $n$ depend on the choice of basis used for the irreps $Z^{\ell}$. We pick an $H$-admissible basis [188], for which each $Z^{\ell}$ decomposes into blocks of some irreps $\lambda(\ell) \in \hat{\mathrm{H}}$ when restricted to $h \in \mathrm{H}$ :

$$
Z^{\ell}(h)=\bigoplus_{\lambda(\ell) \in \hat{\mathrm{H}}} Z^{\lambda(\ell)}(h) .
$$

Picking a particular matrix element $p, n$ selects one of three cases:

(1) a matrix element that is outside of the above block decomposition and, hence, zero;

(2) a matrix element of a nontrivial irrep $\lambda(\ell) \neq \mathbf{1}$; or

(3) a matrix element of the trivial irrep $\lambda(\ell)=\mathbf{1}$.

In case (2), the group orthogonality relations (Table IV E) for $\mathrm{H}$ tell us that such a matrix element averaged over $\mathrm{H}$ is zero [since we assume the irrep is nontrivial and $\left.Z_{00}^{1}(h)=1\right]$. Thus, only case (3) survives, and we see that $\mathrm{H}^{\perp}$ consists of only those irreps $\ell$ that contain a trivial irrep of $\mathrm{H}$. Since the trivial irrep is one dimensional, since we are using an $\mathrm{H}$-admissible basis, and since our average has a $1 /|\mathrm{H}|$ factor, one must have $Z_{p n}^{\ell}(\mathrm{H})=\delta_{p n}$ for all $n$ corresponding to trivial $\lambda(\ell)=\mathbf{1}$ in decomposition (D4). Plugging this result in yields

$$
\begin{aligned}
|a \mathrm{H}\rangle & \left.=\left.\sum_{\ell m n \in \hat{\mathrm{G}}} \sqrt{\frac{d_{\ell}}{|\mathrm{G} / \mathrm{H}|}} \sum_{p=1}^{d_{\ell}} Z_{m p}^{\ell \star}(a) Z_{p n}^{\ell^{\star}}(\mathrm{H})\right|_{m n} ^{\ell}\right\rangle \\
& \left.=\left.\sum_{\ell \in \mathrm{H}^{\perp}} \sum_{n=1}^{d_{\ell}} \sum_{m=1}^{d_{\ell}} \sqrt{\frac{d_{\ell}}{|\mathrm{G} / \mathrm{H}|}} Z_{m n}^{\ell \star}(a) Z_{n n}^{\ell \star}(\mathrm{H})\right|_{m n} ^{\ell}\right\rangle \\
& \left.=\left.\sum_{\ell m n \in \widehat{\mathrm{G} / \mathrm{H}}} \sqrt{\frac{d_{\ell}}{|\mathrm{G} / \mathrm{H}|}} Z_{m n}^{\ell \star}(a \mathrm{H})\right|_{m n} ^{\ell}\right\rangle,
\end{aligned}
$$

where we collect the surviving $\ell \in \mathrm{H}^{\perp}$, the surviving $n$ designated by our $\mathrm{H}$-admissible basis, and all $m \in$ $\left\{1, \ldots, d_{\ell}\right\}$ into $\widehat{\mathrm{G} / \mathrm{H}}$. This set determines the G-momentum states ||$\left._{m n}^{\ell}\right\rangle$ that form the momentum basis for $\mathrm{G} / \mathrm{H}$.

Since the position states $|a \mathrm{H}\rangle$ each consist of different group elements, they are orthogonal. Inserting a resolution of identity in terms of G-momentum states into $\left\langle a \mathrm{H} \mid a^{\prime} \mathrm{H}\right\rangle$ yields completeness relations

$$
\begin{aligned}
\delta_{a a^{\prime}}^{\mathrm{G} / \mathrm{H}} & =\left\langle a \mathrm{H} \mid a^{\prime} \mathrm{H}\right\rangle \\
& =\sum_{\ell m n \in \hat{\mathrm{G}}}\left\langle\left. a \mathrm{H}\right|_{m n} ^{\ell}\right\rangle\left\langle{ }_{m n}^{\ell} \mid a^{\prime} \mathrm{H}\right\rangle \\
& =\sum_{\ell m n \in \widehat{\mathrm{G}} / \mathrm{H}} \frac{d_{\ell}}{|\mathrm{G} / \mathrm{H}|} Z_{m n}^{\ell}(a \mathrm{H}) Z_{m n}^{\ell \star}\left(a^{\prime} \mathrm{H}\right) .
\end{aligned}
$$

Taking two triples from $\widehat{\mathrm{G} / \mathrm{H}}$ and inserting the positionstate identity resolution on $\mathrm{G} / \mathrm{H}$ yields the orthogonality relation

$$
\begin{aligned}
\delta_{\ell \ell^{\prime}} \delta_{m m^{\prime}} \delta_{n n^{\prime}} & =\left\langle{ }_{m n}^{\ell}||_{m^{\prime} n^{\prime}}^{\ell^{\prime}}\right\rangle \\
& =\sum_{a \in \mathrm{G} / \mathrm{H}}\left\langle{ }_{m n}^{\ell} \mid a \mathrm{H}\right\rangle\left\langle\left. a \mathrm{H}\right|_{m^{\prime} n^{\prime}} ^{\ell^{\prime}}\right\rangle \\
& =\frac{d_{\ell}}{|\mathrm{G} / \mathrm{H}|} \sum_{a \in \mathrm{G} / \mathrm{H}} Z_{m n}^{\ell \star}(a \mathrm{H}) Z_{m^{\prime} n^{\prime}}^{\ell^{\prime}}(a \mathrm{H}) .
\end{aligned}
$$

Using Eq. (D5c) and applying the completeness relation yields the Fourier transform on $\mathrm{G} / \mathrm{H}$ :

$$
\sum_{a \in \mathrm{G} / \mathrm{H}} \sqrt{\frac{d_{\ell}}{|\mathrm{G} / \mathrm{H}|}} Z_{m n}^{\ell}(a \mathrm{H})|a \mathrm{H}\rangle=\left|{ }_{m n}^{\ell}\right\rangle .
$$

All position shifts $\vec{X}_{g}$ act in an induced representation (131), but only H-twirled momentum kicks (135) keep one inside $\mathrm{G} / \mathrm{H}$ :

$$
\hat{Z}_{m n}^{\ell}(\mathrm{H})|a \mathrm{H}\rangle=Z_{m n}^{\ell}(a \mathrm{H})|a \mathrm{H}\rangle .
$$

The remaining identities in the second column in Table $\mathrm{V}$ are determined from the above and Table IV.

Denoting $\vec{X}_{g}$ projected onto $\mathrm{G} / \mathrm{H}$ as $\hat{X}_{g}$, the products

$$
\hat{B}_{g}^{\ell m n}=\sqrt{\frac{d_{\ell}}{|\mathrm{G}|}} \hat{Z}_{m n}^{\ell}(\mathrm{H}) \hat{X}_{g}
$$

for $g \in \mathrm{G}$ and $\underset{m n}{\ell} \in \widehat{\mathrm{G} / \mathrm{H}}$ form an overcomplete frame for operators acting on $\mathrm{G} / \mathrm{H}$ : 


$$
\sum_{g \in \mathrm{G}} \sum_{\ell m n \in \widehat{\mathrm{G} / \mathrm{H}}}\left\langle a \mathrm{H}\left|\hat{B}_{g}^{\ell m n}\right| b \mathrm{H}\right\rangle\left\langle b^{\prime} \mathrm{H}\left|\hat{B}_{g}^{\ell m n^{\dagger}}\right| a^{\prime} \mathrm{H}\right\rangle=\delta_{a a^{\prime}}^{\mathrm{G} / \mathrm{H}} \delta_{b b^{\prime}}^{\mathrm{G} / \mathrm{H}},
$$

for all $a, a^{\prime}, b, b^{\prime} \in \mathrm{F}_{\mathrm{G} / \mathrm{H}}$. This formula can be obtained by using the group orthogonality relations and noting that

$$
\sum_{g \in \mathrm{G}} \hat{X}_{g}|a \mathrm{H}\rangle\left\langle a \mathrm{H}\left|\hat{X}_{g}^{\dagger}=\hat{X}_{a} \sum_{g \in \mathrm{G}}\right| g \mathrm{H}\right\rangle\left\langle g \mathrm{H}\left|\hat{X}_{a}^{\dagger}=\right| \mathrm{H}\right| 1_{\mathrm{G} / \mathrm{H}} .
$$

Some of the other position shifts $\overleftarrow{X}_{g}$ remain unitary when projected onto $\mathrm{G} / \mathrm{H}$, as we shortly see in the examples below.

\section{Example: Rigid rotor}

Recall from Appendix $\mathrm{B}$ that the rigid rotor is itself a quotient space, $\mathrm{SO}_{3}=\mathrm{SU}_{2} / \mathrm{Z}_{2}$, where $\mathrm{Z}_{2}=\{I,-I\}$. We can interpret it as a subspace of $\mathrm{SU}_{2}$, with position states

$$
|R\rangle=\frac{1}{\sqrt{2}}\left(|+R\rangle_{\mathrm{SU}_{2}}+|-R\rangle_{\mathrm{SU}_{2}}\right),
$$

where $\mathrm{R} \in \mathrm{SO}_{3}$ and $| \pm R\rangle_{\mathrm{SU}_{2}}$ are position states in $\mathrm{SU}_{2}$. To determine the $\mathrm{SU}_{2}$ momentum states $\left|{ }_{m n}^{\ell}\right\rangle$ (with $\ell \in \mathrm{Z} / 2$ now being integer or half-integer) participating in the momentum basis of $\mathrm{SO}_{3}$, we can expand $| \pm R\rangle_{\mathrm{SU}_{2}}$ in terms of momentum states and simplify the sum (i.e., $\mathrm{Z}_{2}$ twirl). The irreps of $\mathrm{SU}_{2}$ are also expressible in terms of Wigner $D$ matrices, and such $\mathrm{Z}_{2}$ twirls are simple:

$$
\begin{aligned}
\sqrt{\frac{d_{\ell}}{\left|\mathrm{SU}_{2}\right| /\left|\mathrm{Z}_{2}\right|}} D^{\ell}\left(R \mathrm{Z}_{2}\right) & =\sqrt{\frac{2 \ell+1}{8 \pi^{2}}} \frac{1}{2}\left[D^{\ell}(R)+D^{\ell}(-R)\right] \\
& =\delta_{\ell \in \mathrm{Z}} \sqrt{\frac{2 \ell+1}{8 \pi^{2}}} D^{\ell}(R),
\end{aligned}
$$

since $D^{\ell}(-R)=-D^{\ell}(R)$ for the half-integer irreps. This result shows that only the integer $\mathrm{SU}_{2}$ irreps participate in $\mathrm{SO}_{3}$. The momentum states of $\mathrm{SO}_{3}$ are, thus, $\left|{ }_{m n}^{\ell}\right\rangle$ with $0 \leq|m|,|n| \leq \ell$.

The $\vec{X}_{R}, \widehat{X}_{R}$, and $\hat{D}_{m n}^{\ell}$ of the rigid rotor (see Sec. V) are inherited directly from their analogs on $\mathrm{SU}_{2}$. The $\vec{X}_{R}$ and $\overleftarrow{X}_{R}$ operators together form the group $\mathrm{SO}_{3} \times \mathrm{SO}_{3}$, the joint group of lab-frame and molecule-frame transformations for an asymmetric molecule.

\section{Example: Linear rotor}

A canonical example of a coset space is the two-sphere, where $\mathrm{G}=\mathrm{SO}_{3}$ and $\mathrm{H}=\mathrm{U}_{1}$. Here, we show that this space is equivalent to an appropriately chosen subspace of the rigid rotor $\mathrm{SO}_{3}$. Picking $\mathrm{U}_{1}$ to be $\mathbf{z}$-axis $\mathrm{SO}_{3}$ rotations $R_{00 \gamma}$ (in the Euler angle $\phi \theta \gamma$ parameterization), $\mathbf{v}=(\theta, \phi) \in \mathrm{S}^{2}$, and the Wigner $D$ matrices [145] are already in a $\mathrm{U}_{1}$ admissible basis. The position states are then

$$
|\mathbf{v}\rangle \equiv \frac{1}{\sqrt{2 \pi}} \int_{\mathrm{U}_{1}} d \gamma\left|R_{\phi \theta \gamma}\right\rangle
$$

To determine the momentum states $\left|{ }_{m n}^{\ell}\right\rangle$ participating in the momentum basis of $\mathrm{S}^{2}$, we can expand $\left|R_{\phi \theta \gamma}\right\rangle$ in terms of momentum states and perform the $U_{1}$ integral (i.e., twirl) of the corresponding coefficients $D_{m n}^{\ell}$. Using Sec. 5.2.7 in Ref. [109] yields

$$
\begin{aligned}
\sqrt{\frac{d_{\ell}}{\left|\mathrm{SO}_{3}\right| /\left|\mathrm{U}_{1}\right|}} D_{m n}^{\ell}\left(\mathbf{v \mathrm { U } _ { 1 }}\right) & =\sqrt{\frac{2 \ell+1}{4 \pi}} \frac{1}{2 \pi} \int_{\mathrm{U}_{1}} d \gamma D_{m n}^{\ell}(\phi, \theta, \gamma) \\
& =\delta_{n 0} Y_{m}^{\ell}(\theta, \phi) .
\end{aligned}
$$

Thus, the $S^{2}$ momentum states are ||$\left.\left._{m}^{\ell}\right\rangle \equiv||_{m 0}^{\ell}\right\rangle$. Further applying this machinery works out the rest of the third column in Table V.

In this framework, the $X$ - (98) and $Z$-type (99) operators of $S^{2}$ can be viewed as projections of the $X$ - and $Z$-type operators of $\mathrm{SO}_{3}$ onto $\mathrm{S}^{2}$. From Table IV F, we see that all $\vec{X}_{R}$ for $R \in \mathrm{SO}_{3}$ are also operators on $\mathrm{S}^{2}$, acting on the $m$ indices of the $S^{2}$ momentum states and yielding the position shifts $\hat{X}_{R}$. Projecting the $\overleftarrow{X}_{R}$ operators, on the other hand, retains only certain matrix elements:

$$
\left\langle{ }_{m}^{\ell}\left|\overleftarrow{X}_{\alpha \beta \gamma}\right|_{m^{\prime}}^{\ell^{\prime}}\right\rangle=\delta_{\ell \ell^{\prime}} \delta_{m m^{\prime}} D_{00}^{\ell}(0 \beta 0) .
$$

Only two values $\beta \in\{0, \pi\}$ yield unitary operators on $S^{2}$, with $\beta=0$ being the identity and $\beta=\pi$ being the $S^{2}$ inversion operation $\hat{X}_{P}$. These form the group $\mathrm{Z}_{2}^{P}$, which together with the projected $\vec{X}_{R}$ 's forms the group $\mathrm{SO}_{3} \times$ $\mathrm{Z}_{2}^{P}=\mathrm{O}_{3}$ of proper and improper rotations on $\mathrm{S}^{2}$.

\section{Example: $Z_{N}$-symmetric rotor}

Another space of interest is $\mathrm{SO}_{3} / \mathrm{Z}_{N}$, the orientation space of a $Z_{N}$-symmetric molecule. Using $\mathbf{z}$-axis rotations for $Z_{N}$, this space is a subspace of $\mathrm{SO}_{3}$ with position states

$$
|a\rangle=\frac{1}{\sqrt{N}} \sum_{h \in Z_{N}}\left|R_{\phi, \theta, \xi+(2 \pi / N) h}\right\rangle,
$$

where $a=(\phi, \theta, \xi) \in \mathrm{F}_{\mathrm{SO}_{3} / \mathrm{Z}_{N}},(\theta, \phi) \in \mathrm{S}^{2}$, and $\xi \in[0,2 \pi / N)$. To determine the participating momentum states, we perform the $\mathrm{Z}_{N}$ twirl

$$
\sqrt{\frac{d_{\ell}}{\left|\mathrm{SO}_{3}\right| /\left|\mathrm{Z}_{N}\right|}} D_{m n}^{\ell}\left(a \mathrm{Z}_{N}\right)=\delta_{n 0}^{\mathrm{Z}_{N}} \sqrt{\frac{2 \ell+1}{8 \pi^{2} / N}} D_{m n}^{\ell}(a),
$$


where $\delta_{n m}^{Z_{N}}=1$ if $m=n$ modulo $N$. Thus, the set of momentum states is

$$
\left.\left\{||_{m, N p}^{\ell}\right\rangle, 0 \leq|N p|,|m| \leq \ell\right\} .
$$

As with the linear rotor, this space inherits all $\vec{X}_{R}$ rotations. However, projecting the $\overleftarrow{X}_{R}$ rotations yields

$$
\left\langle{ }_{m, N p}^{\ell}\left|\overleftarrow{X}_{\alpha \beta \gamma}\right|_{m^{\prime}, N p^{\prime}}^{\ell^{\prime}}\right\rangle=\delta_{\ell \ell^{\prime}} \delta_{m m^{\prime}} D_{N p, N p^{\prime}}^{\ell}(\alpha \beta \gamma) .
$$

As opposed to the case of the linear rotor, $\overleftarrow{X}_{R}$ is not diagonal in the momentum basis, and so there are more unitary operators inherited from such rotations. For example, all triples $(\alpha 0 \gamma)$ and $(\alpha \pi \gamma)$ yield unitary operators. For $|p|,\left|p^{\prime}\right|=1$, these form the group $\mathrm{O}_{2} / \mathrm{Z}_{N}$. Together with all $\vec{X}_{R}$ 's, these form the group $\mathrm{SO}_{3} \times \mathrm{O}_{2} / \mathrm{Z}_{N}$.

As $N \rightarrow \infty, \xi \rightarrow 0$ and this space approaches $\mathrm{S}^{2}$. In pictures, the saucerlike space [see Fig. 2(b)] compresses to a flat pancake with all of its boundary points identified, which is equivalent to the two-sphere.

\section{Poisson summation}

A final interesting note is the presence of a Poisson summation formula on these spaces. Recall the standard formula for functions $f \in \mathcal{L}^{2}(\mathrm{R})$ :

$$
\sum_{h \in \mathrm{Z}} f(h)=\sum_{\ell \in \mathrm{Z}} \int_{\mathrm{R}} d x e^{i \ell x} f(x) .
$$

Oftentimes, the first term in the sum over $\ell$ is sufficient asymptotically with some parameter (see Sec. V C), so this formula is useful to approximate sums with integrals. A closer inspection reveals that this formula is a special case $(\mathrm{H}=\mathrm{Z}$ and $\mathrm{G}=\mathrm{R})$ of the more general formula for evaluating the sum of a function $f \in \mathcal{L}^{2}(\mathrm{G})$ over $\mathrm{H}$ [127]:

$$
\begin{aligned}
\frac{1}{|\mathrm{H}|} \sum_{h \in \mathrm{H}} f(h) & =\frac{1}{\sqrt{|\mathrm{H}|}}\langle\mathrm{H} \mid f\rangle \\
& =\sum_{\ell m \in \widehat{\mathrm{G} / \mathrm{H}}} \sqrt{\frac{d_{\ell}}{|\mathrm{G}|}} \sum_{g \in \mathrm{G}}\left\langle{ }_{m m} \mid g\right\rangle\langle g \mid f\rangle \\
& =\sum_{\ell m \in \widehat{\mathrm{G} / \mathrm{H}}} \frac{d_{\ell}}{|\mathrm{G}|} \sum_{g \in \mathrm{G}} Z_{m m}^{\ell \star}(g) f(g) .
\end{aligned}
$$

This formula can be generalized to sums over cosets $a \mathrm{H}$.

\section{APPENDIX E: PARTIAL FOURIER TRANSFORM ON $\mathrm{S}^{2}$}

Our focus here is on protection against small rotations, which necessitates the use of coherences between antipodal orientations to store the logical information [i.e., satisfaction of Eq. (107)]. Given this condition, it is convenient to let the subgroup $\mathrm{K} \supset \mathrm{H}$ be $\mathrm{H} \times \mathrm{Z}_{2}^{P}$, where $\mathrm{Z}_{2}^{P}$ is the group generated by inversion $P$. Since there are only two cosets $\{\mathrm{H}, P \mathrm{H}\}$ of $\mathrm{H}$ in $\mathrm{K}$, our choice of $\mathrm{K}$ restricts us to only qubit codes. (Other choices for $\mathrm{K}$ are, of course, possible, but we do not expound on them here.)

\section{Abelian subgroup codes}

Let us pick $\mathrm{H}=\mathrm{Z}_{N}$ (for odd $N$ ) and $\mathrm{K}=\mathrm{Z}_{N} \times \mathrm{Z}_{2}^{P}$, where $\mathrm{Z}_{N}$ corresponds to the group of $\mathbf{z}$-axis rotations. The orbit of a point $(\vartheta, \varphi)$ (with $\vartheta \notin\{0, \pi\}$ ) under K consists of the $2 N$ points $[\vartheta, \varphi+(\pi / N) h]$ and $[\pi-\vartheta, \varphi+(\pi / N) h+$ $\pi]$ with $h \in\{0,1, \ldots, N-1\}$. To form our basis, these points are then split up into two sets of $N$ points, each set corresponding to one of the two cosets labeled by $r \in\{0,1\}$. The two cosets are then Fourier transformed to construct the respective basis states $\{r, \lambda\}$ (with $\lambda \in\{0,1, \ldots, N-1\})$ for each orbit $(\vartheta, \varphi)$, yielding the basis

$$
\begin{aligned}
& \left|0 \mathrm{Z}_{N}(\vartheta, \varphi) ; \lambda\right\rangle=\frac{1}{\sqrt{N}} \sum_{h \in Z_{N}} e^{i(2 \pi / N) \lambda h}\left|\vartheta, \varphi+\frac{2 \pi}{N} h\right\rangle, \quad \text { (E1a) } \\
& \left|1 \mathrm{Z}_{N}(\vartheta, \varphi) ; \lambda\right\rangle=\frac{1}{\sqrt{N}} \sum_{h \in Z_{N}} e^{i(2 \pi / N) \lambda h}\left|\pi-\vartheta, \varphi+\frac{2 \pi}{N} h+\pi\right\rangle .
\end{aligned}
$$

These states are defined for all $(\vartheta, \varphi)$ belonging to the Voronoi cell of $|(\pi / 2), 0\rangle$ :

$\mathrm{F}_{\mathrm{S}^{2} /\left(\mathrm{Z}_{N} \times \mathrm{Z}_{2}^{P}\right)}=\left\{(\vartheta, \varphi) \mid \vartheta \in[0, \pi], \varphi \in\left(-\frac{\pi}{2 N}, \frac{\pi}{2 N}\right]\right\}$,

except at the points $\vartheta \notin\{0, \pi\}$. This cell is depicted by the blue spherical line in Fig. 2(c). The code words (102) correspond to $\left|0 Z_{N}(\pi / 2,0) ; 0\right\rangle$ and $\left|1 Z_{N}(\pi / 2,0) ; 0\right\rangle$, respectively. The cone points $\vartheta \in\{0, \pi\}$ are special in that they are invariant under any $\mathrm{Z}_{N}$ rotations around the $\mathbf{z}$ axis. For such points, $\lambda=0$, and their orbits under $\mathrm{Z}_{N}$ are simply the points themselves, $\left|0 \mathrm{Z}_{N}(0,0) ; \lambda\right\rangle=$ $|0,0\rangle$ and $\left|1 Z_{N}(\pi, 0) ; \lambda\right\rangle=|\pi, 0\rangle$.

The above basis is orthonormal and complete due to Eq. (112), and, with this basis at hand, we can devise a recovery map for our code. A simple map consists of isometries mapping the subspace $\left\{\left|r Z_{N}(\vartheta, \varphi) ; \lambda\right\rangle\right\}_{r \in\{0,1\}}$ for each $(\vartheta, \varphi)$ and $\lambda$ (with $\vartheta \notin\{0, \pi\}$ ) into the code space $\{|\bar{r}\rangle\}_{r \in\{0,1\}}$. The remaining cone points $\vartheta=0, \pi$ can be mapped to any state in the code space, and we choose to map them to $(1 / \sqrt{2})(|\overline{0}\rangle+|\overline{1}\rangle)$.

Because it preserves coherences between antipodal points, this recovery protects from all rotations $R$ that keep each orientation in its Voronoi cell. To see this protection, 
consider $N=3$ and write a general code state (with $\left.\left|c_{0}\right|^{2}+\left|c_{1}\right|^{2}=1\right)$ as

$$
|\psi\rangle=c_{0}|\overline{0}\rangle+c_{1}|\overline{1}\rangle=\frac{1}{\sqrt{3}}\left(\left|\psi_{0}\right\rangle+\left|\psi_{1}\right\rangle+\left|\psi_{2}\right\rangle\right),
$$

where, for $h \in\{0,1,2\}$, the states

$$
\left|\psi_{h}\right\rangle=c_{0}\left|\frac{\pi}{2}, \frac{2 \pi}{N} h\right\rangle+c_{1}\left|\frac{\pi}{2}, \frac{2 \pi}{N} h+\pi\right\rangle
$$

are superpositions of a pair of antipodal points. These states are mapped to $\hat{X}_{R}\left|\psi_{h}\right\rangle\left\langle\psi_{h}\right| \hat{X}_{R}^{\dagger}$ upon a rotation $\hat{X}_{R}$, with each constituent orientation $|(\pi / 2),(2 \pi / N) h\rangle$ being mapped to some point $\left|\mathbf{v}_{h}\right\rangle$ and its antipode to $\left|-\mathbf{v}_{h}\right\rangle$. Each $\left|\mathbf{v}_{h}\right\rangle$ is supported on $\left|0 \mathrm{Z}_{N}\left(\vartheta_{h}, \varphi_{h}\right) ; \lambda\right\rangle$ for all $\lambda$ and some $\vartheta_{h}, \varphi_{h}$, and similarly $\left|-\mathbf{v}_{h}\right\rangle$ overlaps with $\left|1 Z_{N}\left(\vartheta_{h}, \varphi_{h}\right) ; \lambda\right\rangle$ for all $\lambda$. Our recovery maps each $\hat{X}_{R}\left|\psi_{h}\right\rangle\left\langle\psi_{h}\right| \hat{X}_{R}^{\dagger}$ back into the code space, preserving the logical information. Coherences $\left|\psi_{h}\right\rangle\left\langle\psi_{h^{\prime} \neq h}\right|$ are not preserved, but this result is not detrimental, since the logical information is already inside each $\left|\psi_{h}\right\rangle$.

\section{Non-Abelian subgroups}

Here, we construct the partial Fourier-transformed basis for $\mathrm{H} \subset \mathrm{K}$, where, for simplicity, we assume $\mathrm{H}$ to be the maximal subgroup of $\mathrm{K}$. By identifying points connected by actions of rotations in $\mathrm{K}, \mathrm{S}^{2}$ can be partitioned into orbits $\mathrm{Kw}=\{|k \mathbf{w}\rangle\}_{k \in \mathrm{K}}$ with $\mathbf{w} \in \mathrm{S}^{2} / \mathrm{K}$.

Since $S^{2}$ is not a group, the number of points in an orbit $\mathrm{K} \mathbf{w}$ depends on the starting point $\mathbf{w} \in \mathrm{S}^{2}$. Generically, each rotation $R \in \mathrm{K}$ maps $\mathbf{w}$ to a distinct point $R \mathbf{w}$, but there exist special points (e.g., the aforementioned cone points), invariant under some (or even all) $R$, for which the size of the orbit is $<|\mathrm{K}|$. We have to consider such complications when designing the partial Fourier-transformed basis on $\mathrm{S}^{2}$.

We now further partition each $\mathrm{Kw}$ into one or more parts, corresponding to cosets of $\mathrm{H}$ in $\mathrm{K}$. To do so, we apply the orbit-stabilizer theorem for each orbit. Consider the subset $\mathrm{Hw}$ for each orbit $\mathrm{Kw}$, whose maximal invariant group is either $\mathrm{H}$ or $\mathrm{K}$ (as there are no subgroups in between). If this group is $\mathrm{H}$, then by the theorem there is a one-to-one correspondence between elements of the orbit of $\mathrm{Hw}$ under $\mathrm{K}$ and cosets $a \in \mathrm{K} / \mathrm{H}$. If the group is $\mathrm{K}$, then $\mathrm{Hw}=\mathrm{Kw}$.

Applying the $\mathrm{H}$ Fourier transform on each subset $a \mathrm{Hw}=$ $\{|a R \mathbf{w}\rangle\}_{R \in \mathrm{H}}$ yields (cf. [127])

$$
\left|a \mathrm{H} \mathbf{w}_{\mu \nu}^{\lambda(\mathbf{w})}\right\rangle=\frac{\sqrt{|\mathrm{Hw}|}}{|\mathrm{H}|} \sum_{R \in \mathrm{H}} Z_{\mu \nu}^{\lambda(\mathbf{w})}(R)|a R \mathbf{w}\rangle,
$$

indexed by orbits $\mathbf{w} \in \mathrm{S}^{2} / \mathrm{K}$, cosets $a \in \mathrm{K} / \mathrm{H}$, and irrep elements ${ }_{\mu \nu}^{\lambda}(\mathbf{w}) \in \hat{\mathrm{H}}$. The irrep elements depend on $\mathbf{w}$, since the size $|\mathrm{Hw}|$ of each orbit, and, therefore, the number of states in the subspace $\{|R \mathbf{w}\rangle\}_{R \in \mathrm{H}}$, depends on $\mathbf{w}$. Likewise, the coset index $a$ is used only when $|\mathrm{Kw}| \neq|\mathrm{Hw}|$; otherwise, $\mathrm{Hw}$ is invariant under $\mathrm{K}$, and no $a$ index is needed.

A simple example is $\mathrm{H}=\mathrm{T}$ and $\mathrm{K}=\mathrm{T} \times \mathrm{Z}_{2}^{P}$. A generic orbit $a \mathrm{Tw}$ is a tetrahedrally symmetric set of 12 points, and $\mathrm{Kw}$ is an octahedrally symmetric set of 24 points. At a special point $\mathbf{w}_{\text {cube }}, T \mathbf{w}_{\text {cube }}$ form the vertices of a tetrahedron, and $\mathbf{K w}_{\text {cube }}$ is a cube (Fig. 5). We pick the corresponding two states $\left\{\left|a \mathrm{Tw}_{\text {cube }} ;{ }_{00}\right\rangle\right\}_{a \in \mathrm{Z}_{2}^{p}}$ to be the code words (where $\mathbf{1}$ is the trivial irrep of T).

\section{Symmetric harmonics}

A simple way to obtain K-symmetric harmonics from the spherical harmonics is to average or twirl them over $\mathrm{K}$ :

$$
\hat{Y}_{m}^{\ell}(\mathrm{K}) \equiv \frac{1}{|\mathrm{~K}|} \sum_{k \in \mathrm{K}} \hat{X}_{k} \hat{Y}_{m}^{\ell} \hat{X}_{k}^{\dagger}=\sum_{|p| \leq \ell} D_{p m}^{\ell \star}(\mathrm{K}) \hat{Y}_{p}^{\ell}
$$

Above, we use Eq. (128), the Weyl relation (Table V H), and Sec. 5.5.2, Eq. (1), in Ref. [109] to express the twirl in terms of Wigner $D$ matrices [145]. The above is nonzero only for those $\ell$ admitting K-symmetric harmonics.

\section{APPENDIX F: BROADER CONTEXT}

The partially Fourier-transformed basis (20) for $\mathrm{Z}_{3} \subset \mathrm{U}_{1}$ and its generalization (124) for subgroups $\mathrm{H} \subset \mathrm{G}$ are prominent in many areas of science and engineering. We list notable examples and three interpretations below.

\section{Notable examples}

A particularly famous example is lattice systems $\{\mathrm{G}, \mathrm{H}\}=\left\{\mathrm{R}^{\times d}, \mathrm{Z}^{\times d}\right\}$, where

$$
\mathrm{R}^{\times d} \cong(\mathrm{R} / \mathrm{Z})^{\times d} \times \widehat{\mathrm{Z}^{\times d}} \cong \mathrm{U}_{1}^{\times d} \times \mathrm{U}_{1}^{\times d} .
$$

The corresponding basis (124) is called the Weil-Brezin transform [see Eq. (1.112) in Ref. [191]] or, in the solidstate context, the Zak or $k q$ basis [192]: The first $\mathrm{U}_{1}^{\times d}$ factor is parameterized by angles replacing the discrete band index $n$ in the standard Bloch functions, while the second factor is simply $k$ space. This basis sees applications in signal processing [193], where it is useful for resolving signals from noise. This basis is also studied in quantum foundations [194], and its constituent states can be grouped to form a code space and error spaces for GKP codes [1] (a motivation for this work).

Another interesting example is $\left\{\mathrm{SO}_{3}, \mathrm{U}_{1}\right\}$. Its corresponding decomposition

$$
\mathrm{SO}_{3} \cong \mathrm{SO}_{3} / \mathrm{U}_{1} \times \widehat{\mathrm{U}_{1}} \cong \mathrm{S}^{2} \times \mathrm{Z}
$$

is useful for expressing vector fields on the sphere (see Sec. 12.3 in Ref. [195]). 


\section{Symmetry-adapted bases}

Such bases are used to block-diagonalize H-symmetric Hamiltonians acting on a space $\mathrm{X}$ into blocks corresponding to irreps $\lambda \in \hat{\mathrm{H}}$. For the $\left\{\mathrm{U}_{1}, \mathrm{Z}_{3}\right\}$ example, a $\mathrm{Z}_{3^{-}}$ symmetric Hamiltonian written in the basis (20) does not have any matrix elements connecting different values of $\lambda$. This diagonalization procedure is ubiquitous in physics and chemistry (see Secs. 3-8 in Ref. [190]) (see also Ref. [127]).

\section{Coherent states}

The states $\left\{\left|a Z_{3} ; \lambda\right\rangle\right\}_{a \in \mathrm{U}_{1} / \mathrm{Z}_{3}}$ for fixed $\lambda$ can then be obtained by applying position shifts on the fiducial state $\left|0 Z_{3} ; \lambda\right\rangle$, making them similar to Perelomov coherent states (see Sec. II. 1 in Ref. [99]) (with the caveat that the representation of the group of shifts is reducible). A key difference between such states for $\{\mathrm{R}, \mathrm{Z}\}$ and the conventional oscillator coherent states is the choice of fiducial state: a GKP state for the former and the vacuum Fock state for the latter.

\section{Fiber bundles}

If we instead take a look at $\left\{\left|a Z_{3} ; \lambda\right\rangle\right\}_{\lambda \in Z_{3}}$, we have a 3D space for each $a \in \mathrm{U}_{1} / \mathrm{Z}_{3}$. For this and any $\{\mathrm{G}, \mathrm{H}\}$ case where $\mathrm{G}$ is a Lie group, the states form a fiber bundle with base space $\mathrm{G} / \mathrm{H}$, fiber $\hat{\mathrm{H}}$, and cross section $\{a\}$ [99].

[1] D. Gottesman, A. Yu. Kitaev, and J. Preskill, Encoding a Qubit in an Oscillator, Phys. Rev. A 64, 012310 (2001).

[2] B. M. Terhal and D. Weigand, Encoding a Qubit into a Cavity Mode in Circuit QED Using Phase Estimation, Phys. Rev. A 93, 012315 (2016).

[3] V. V. Albert, K. Noh, K. Duivenvoorden, D. J. Young, R. T. Brierley, P. Reinhold, C. Vuillot, L. Li, C. Shen, S. M. Girvin, B. M. Terhal, and L. Jiang, Performance and Structure of Single-Mode Bosonic Codes, Phys. Rev. A 97, 032346 (2018).

[4] K. Noh, V. V. Albert, and L. Jiang, Quantum Capacity Bounds of Gaussian Thermal Loss Channels and Achievable Rates with Gottesman-Kitaev-Preskill Codes, IEEE Trans. Inf. Theory 65, 2563 (2019).

[5] C. Flühmann, T. L. Nguyen, M. Marinelli, V. Negnevitsky, K. Mehta, and J. P. Home, Encoding a Qubit in a TrappedIon Mechanical Oscillator, Nature (London) 566, 513 (2019).

[6] P. Campagne-Ibarcq, A. Eickbusch, S. Touzard, E. ZalysGeller, N. E. Frattini, V. V. Sivak, P. Reinhold, S. Puri, S. Shankar, R. J. Schoelkopf, L. Frunzio, M. Mirrahimi, and M.H. Devoret, Quantum Error Correction of a Qubit Encoded in Grid States of an Oscillator, Nature (London) 584, 368 (2020).

[7] M. Zeppenfeld, B. G. U. Englert, R. Glöckner, A. Prehn, M. Mielenz, C. Sommer, L. D. van Buuren, M. Motsch, and G. Rempe, Sisyphus Cooling of Electrically Trapped Polyatomic Molecules, Nature (London) 491, 570 (2012).
[8] X. Wu, T. Gantner, M. Koller, M. Zeppenfeld, S. Chervenkov, and G. Rempe, A Cryofuge for ColdCollision Experiments with Slow Polar Molecules, Science 358, 645 (2017).

[9] P. Puri, M. Mills, C. Schneider, I. Simbotin, J. A. Montgomery, R. Côté, A. G. Suits, and E. R. Hudson, Synthesis of Mixed Hypermetallic Oxide $\mathrm{BaOCa}+$ from Laser-Cooled Reagents in an Atom-Ion Hybrid Trap, Science 357, 1370 (2017).

[10] L. Anderegg, L. W. Cheuk, Y. Bao, S. Burchesky, W. Ketterle, K.-K. Ni, and J. M. Doyle, An Optical Tweezer Array of Ultracold Molecules, Science 365, 1156 (2019).

[11] I. Kozyryev, L. Baum, K. Matsuda, B. L. Augenbraun, L. Anderegg, A. P. Sedlack, and J. M. Doyle, Sisyphus Laser Cooling of a Polyatomic Molecule, Phys. Rev. Lett. 118, 173201 (2017).

[12] L. R. Liu, J. D. Hood, Y. Yu, J. T. Zhang, K. Wang, Y.-W. Lin, T. Rosenband, and K.-K. Ni, Molecular Assembly of Ground-State Cooled Single Atoms, Phys. Rev. X 9, 021039 (2019).

[13] J. P. Covey, L. D. Marco, Ó. L. Acevedo, A. M. Rey, and J. Ye, An Approach to Spin-Resolved Molecular Gas Microscopy, New J. Phys. 20, 043031 (2018).

[14] A. Jamadagni, S. Ospelkaus, L. Santos, and H. Weimer, Quantum Zeno-Based Detection and State Engineering of Ultracold Polar Molecules, arXiv:1906.09263.

[15] C. W. Chou, A. L. Collopy, C. Kurz, Y. Lin, M. E. Harding, P. N. Plessow, T. Fortier, S. Diddams, D. Leibfried, and D. R. Leibrandt, Frequency-Comb Spectroscopy on Pure Quantum States of a Single Molecular Ion, Science 367, 1458 (2020).

[16] Y. Lin, D. R. Leibrandt, D. Leibfried, and C.-w. Chou, Quantum Entanglement between an Atom, and a Molecule, Nature (London) 581, 273 (2020).

[17] D. DeMille, Quantum Computation with Trapped Polar Molecules, Phys. Rev. Lett. 88, 067901 (2002).

[18] C. Lee and E. A. Ostrovskaya, Quantum Computation with Diatomic Bits in Optical Lattices, Phys. Rev. A 72, 062321 (2005).

[19] S. F. Yelin, K. Kirby, and R. Côté, Schemes for Robust Quantum Computation with Polar Molecules, Phys. Rev. A 74, 050301(R) (2006).

[20] P. Rabl and P. Zoller, Molecular Dipolar Crystals as HighFidelity Quantum Memory for Hybrid Quantum Computing, Phys. Rev. A 76, 042308 (2007).

[21] M. Ortner, Y. L. Zhou, P. Rabl, and P. Zoller, Quantum Information Processing in Self-Assembled Crystals of Cold Polar Molecules, Quantum Inf. Process. 10, 793 (2011).

[22] E. Kuznetsova, S. F. Yelin, and R. Côté, An Atom-Molecule Platform for Quantum Computing, Quantum Inf. Process. 10, 821 (2011).

[23] J. A. Blackmore, L. Caldwell, P. D. Gregory, E. M. Bridge, R. Sawant, J. Aldegunde, J. Mur-Petit, D. Jaksch, J. M. Hutson, B. E. Sauer, M. R. Tarbutt, and S. L. Cornish, Ultracold Molecules for Quantum Simulation: Rotational Coherences in CaF and RbCs, Quantum Sci. Technol. 4, 014010 (2018).

[24] K.-K. Ni, T. Rosenband, and D. D. Grimes, Dipolar Exchange Quantum Logic Gate with Polar Molecules, Chem. Sci. 9, 6830 (2018). 
[25] E. R. Hudson and W. C. Campbell, Dipolar Quantum Logic for Freely Rotating Trapped Molecular Ions, Phys. Rev. A 98, 040302(R) (2018).

[26] P. Yu, L. W. Cheuk, I. Kozyryev, and J. M. Doyle, A Scalable Quantum Computing Platform Using SymmetricTop Molecules, New J. Phys. 21, 093049 (2019).

[27] W. C. Campbell and E. R. Hudson, Dipole-Phonon Quantum Logic with Trapped Polar Molecular Ions, arXiv: 1909.02668 [Phys. Rev. Lett. (to be published)].

[28] R. Sawant, J. A. Blackmore, P. D. Gregory, J. Mur-Petit, D. Jaksch, J. Aldegunde, J. M. Hutson, M. R. Tarbutt, and S. L. Cornish, Ultracold Molecules as Qudits, New J. Phys. 22, 013027 (2020).

[29] A. Negretti, P. Treutlein, and T. Calarco, Quantum Computing Implementations with Neutral Particles, Quantum Inf. Process. 10, 721 (2011).

[30] R. Côté, Ultracold Molecules: Their Formation and Application to Quantum Computing, in Quantum Information and Computation for Chemistry, edited by S. Kais (Wiley, New York, 2014), Chap. 14, https://doi.org/ 10.1002/9781118742631.ch14.

[31] J. M. Rost, J. C. Griffin, B. Friedrich, and D. R. Herschbach, Pendular States and Spectra of Oriented Linear Molecules, Phys. Rev. Lett. 68, 1299 (1992).

[32] A. Slenczka, B. Friedrich, and D. Herschbach, Pendular Alignment of Paramagnetic Molecules in Uniform Magnetic Fields, Phys. Rev. Lett. 72, 1806 (1994).

[33] M. Karra, K. Sharma, B. Friedrich, S. Kais, and D. Herschbach, Prospects for Quantum Computing with an Array of Ultracold Polar Paramagnetic Molecules, J. Chem. Phys. 144, 094301 (2016).

[34] C. M. Tesch and R. de Vivie-Riedle, Quantum Computation with Vibrationally Excited Molecules, Phys. Rev. Lett. 89, 157901 (2002).

[35] J. J. Baldoví, S. Cardona-Serra, J. M. Clemente-Juan, L. Escalera-Moreno, A. Gaita-Ariño, and G. Mínguez Espallargas, Quantum Error Correction with Magnetic Molecules, Europhys. Lett. 110, 33001 (2015).

[36] J. Preskill, Quantum Computing in the NISQ Era and beyond, Quantum 2, 79 (2018).

[37] D. Patterson, Method for Preparation and Readout of Polyatomic Molecules in Single Quantum States, Phys. Rev. A 97, 033403 (2018).

[38] E. S. Shuman, J. F. Barry, and D. DeMille, Laser Cooling of a Diatomic Molecule, Nature (London) 467, 820 (2010).

[39] L. Anderegg, B. L. Augenbraun, E. Chae, B. Hemmerling, N. R. Hutzler, A. Ravi, A. Collopy, J. Ye, W. Ketterle, and J. M. Doyle, Radio Frequency Magneto-Optical Trapping of CaF with High Density, Phys. Rev. Lett. 119, 103201 (2017).

[40] S. Truppe, H. J. Williams, M. Hambach, L. Caldwell, N. J. Fitch, E. A. Hinds, B. E. Sauer, and M. R. Tarbutt, Molecules Cooled below the Doppler Limit, Nat. Phys. 13, 1173 (2017).

[41] A. L. Collopy, S. Ding, Y. Wu, I. A. Finneran, L. Anderegg, B. L. Augenbraun, J. M. Doyle, and J. Ye, 3D MagnetoOptical Trap of Yttrium Monoxide, Phys. Rev. Lett. 121, 213201 (2018).

[42] S. A. Moses, J. P. Covey, M. T. Miecnikowski, D. S. Jin, and J. Ye, New Frontiers for Quantum Gases of Polar Molecules, Nat. Phys. 13, 13 (2017).
[43] C. P. Koch, M. Lemeshko, and D. Sugny, Quantum Control of Molecular Rotation, Rev. Mod. Phys. 91, 035005 (2019).

[44] S. A. Moses, J. P. Covey, M. T. Miecnikowski, B. Yan, B. Gadway, J. Ye, and D. S. Jin, Creation of a Low-Entropy Quantum Gas of Polar Molecules in an Optical Lattice, Science 350, 659 (2015).

[45] L. Reichsöllner, A. Schindewolf, T. Takekoshi, R. Grimm, and H.-C. Nägerl, Quantum Engineering of a Low-Entropy Gas of Heteronuclear Bosonic Molecules in an Optical Lattice, Phys. Rev. Lett. 118, 073201 (2017).

[46] J. Fortagh and C. Zimmermann, Magnetic Microtraps for Ultracold Atoms, Rev. Mod. Phys. 79, 235 (2007).

[47] L. Caldwell, H. J. Williams, N. J. Fitch, J. Aldegunde, J. M. Hutson, B. E. Sauer, and M. R. Tarbutt, Long Rotational Coherence Times of Molecules in a Magnetic Trap, Phys. Rev. Lett. 124, 063001 (2020).

[48] C. W. Chou, C. Kurz, D. B. Hume, P. N. Plessow, D. R. Leibrandt, and D. Leibfried, Preparation and Coherent Manipulation of Pure Quantum States of a Single Molecular Ion, Nature (London) 545, 203 (2017).

[49] L. De Marco, G. Valtolina, K. Matsuda, W. G. Tobias, J. P. Covey, and J. Ye, A Degenerate Fermi Gas of Polar Molecules, Science 363, 853 (2019).

[50] B. Neyenhuis, B. Yan, S. A. Moses, J. P. Covey, A. Chotia, A. Petrov, S. Kotochigova, J. Ye, and D. S. Jin, Anisotropic Polarizability of Ultracold Polar 40K87Rb Molecules, Phys. Rev. Lett. 109, 230403 (2012).

[51] J. W. Park, Z.Z. Yan, H. Loh, S. A. Will, and M. W. Zwierlein, Second-Scale Nuclear Spin Coherence Time of Ultracold $23 \mathrm{Na} 40 \mathrm{~K}$ molecules, Science 357, 372 (2017).

[52] F. Seeßelberg, X.-Y. Luo, M. Li, R. Bause, S. Kotochigova, I. Bloch, and C. Gohle, Extending Rotational Coherence of Interacting Polar Molecules in a Spin-Decoupled Magic Trap, Phys. Rev. Lett. 121, 253401 (2018).

[53] I. Kozyryev and N. R. Hutzler, Precision Measurement of Time-Reversal Symmetry Violation with Laser-Cooled Polyatomic Molecules, Phys. Rev. Lett. 119, 133002 (2017).

[54] M. V. Ivanov, F. H. Bangerter, and A. I. Krylov, Towards a Rational Design of Laser-Coolable Molecules: Insights from Equation-of-Motion Coupled-Cluster Calculations, Phys. Chem. Chem. Phys. 21, 19447 (2019).

[55] M. L. Wall, K. Maeda, and L. D. Carr, Simulating Quantum Magnets with Symmetric Top Molecules, Ann. Phys. (Amsterdam) 525, 845 (2013).

[56] M. L. Wall, K. Maeda, and L. D. Carr, Realizing Unconventional Quantum Magnetism with Symmetric Top Molecules, New J. Phys. 17, 025001 (2015).

[57] M. J. O'Rourke and N. R. Hutzler, Hypermetallic Polar Molecules for Precision Measurements, Phys. Rev. A 100, 022502 (2019).

[58] I. Kozyryev, L. Baum, K. Matsuda, and J. M. Doyle, Proposal for Laser Cooling of Complex Polyatomic Molecules, Chem. Phys. Chem. 17, 3641 (2016).

[59] B. L. Augenbraun, J. M. Doyle, T. Zelevinsky, and I. Kozyryev, Molecular Asymmetry and Optical Cycling: Laser Cooling Asymmetric Top Molecules, Phys. Rev. X 10, 031022 (2020). 
[60] J. M. Brown and A. Carrington, Rotational Spectroscopy of Diatomic Molecules (Cambridge University Press, Cambridge, England, 2003), https://doi.org/10.1017/ CBO9780511814808.

[61] R. V. Krems, Molecules in Electromagnetic Fields: From Ultracold Physics to Controlled Chemistry (Wiley, New York, 2018), https://www.wiley.com/en-us/ Molecules+in+Electromagnetic+Fields\%3A+From +Ultracold+Physics+to+Controlled+Chemistry-p9781118173619.

[62] W. Demtroder, Molecular Physics (Wiley, New York, 2005), https://doi.org/10.1002/9783527618095.

[63] P. E. S. Wormer, The Rigid Rotor in Classical and Quantum Mechanics (online notes), http://www.theochem.ru.nl/ $\sim$ pwormer/teachmat/rigrotor.pdf.

[64] The actual Hamiltonian depends on the molecule's moments of inertia [60-63]. We use the "spherical top" Hamiltonian to simplify the analysis.

[65] A. V. Gorshkov, K. R. A. Hazzard, and A. M. Rey, Kitaev Honeycomb and Other Exotic Spin Models with Polar Molecules, Mol. Phys. 111, 1908 (2013).

[66] S. R. Manmana, E. M. Stoudenmire, K. R. A. Hazzard, A. M. Rey, and A. V. Gorshkov, Topological Phases in Ultracold Polar-Molecule Quantum Magnets, Phys. Rev. B 87, 081106(R) (2013).

[67] B. Sundar, B. Gadway, and K. R. A. Hazzard, Synthetic Dimensions in Ultracold Polar Molecules, Sci. Rep. 8, 3422 (2018).

[68] A. V. Gorshkov, S. R. Manmana, G. Chen, E. Demler, M. D. Lukin, and A. M. Rey, Quantum Magnetism with Polar Alkali-Metal Dimers, Phys. Rev. A 84, 033619 (2011).

[69] B. Yan, S. A. Moses, B. Gadway, J. P. Covey, K. R. A. Hazzard, A. M. Rey, D. S. Jin, and J. Ye, Observation of Dipolar Spin-Exchange Interactions with Lattice-Confined Polar Molecules, Nature (London) 501, 521 (2013).

[70] T. Rosenband, D. D. Grimes, and K.-K. Ni, Elliptical Polarization for Molecular Stark Shift Compensation in Deep Optical Traps, Opt. Express 26, 19821 (2018).

[71] R. de Vivie-Riedle and U. Troppmann, Femtosecond Lasers for Quantum Information Technology, Chem. Rev. 107, 5082 (2007).

[72] U. Boscain, M. Caponigro, T. Chambrion, and M. Sigalotti, A Weak Spectral Condition for the Controllability of the Bilinear Schrödinger Equation with Application to the Control of a Rotating Planar Molecule, Commun. Math. Phys. 311, 423 (2012).

[73] A. Magann, T.-S. Ho, and H. Rabitz, Singularity-Free Quantum Tracking Control of Molecular Rotor Orientation, Phys. Rev. A 98, 043429 (2018).

[74] D. Zhdanov and H. Rabitz, Laser-Assisted Molecular Alignment in a Strongly Dissipative Environment, Phys. Rev. A 83, 061402(R) (2011).

[75] U. Boscain, M. Caponigro, and M. Sigalotti, MultiInput Schrödinger Equation: Controllability, Tracking, and Application to the Quantum Angular Momentum, J. Differ. Eq. 256, 3524 (2014).

[76] R. Tehini, K. Hamraoui, and D. Sugny, Shaping of the Time Evolution of Field-Free Molecular Orientation by THz Laser Pulses, Phys. Rev. A 99, 033419 (2019).
[77] U. Boscain, E. Pozzoli, and M. Sigalotti, Classical and Quantum Controllability of a Rotating 3D Symmetric Molecule, arXiv:1910.01924.

[78] A. Ma, A. B. Magann, T.-S. Ho, and H. Rabitz, Optimal Control of Coupled Quantum Systems Based on the FirstOrder Magnus Expansion: Application to Multiple DipoleDipole-Coupled Molecular Rotors, Phys. Rev. A 102, 013115 (2020).

[79] Z.-Y. Zhang, J.-M. Liu, Z. Hu, and Y. Wang, Optical Control of Entanglement and Coherence for Polar Molecules in Pendular States, Opt. Express 27, 26588 (2019).

[80] M. Lemeshko and R. Schmidt, Molecular Impurities Interacting with a Many-Particle Environment: From Helium Droplets to Ultracold Gases, arXiv:1703.06753.

[81] A. F. Devonshire, The Rotation of Molecules in Fields of Octahedral Symmetry, Proc. R. Soc. A 153, 601 (1936).

[82] T. Kiljunen, B. Schmidt, and N. Schwentner, Intense-Field Alignment of Molecules Confined in Octahedral Fields, Phys. Rev. Lett. 94, 123003 (2005).

[83] G. K. Pandey, K. L. Pandey, M. Massey, and R. Kumar, Possibility of the Simultaneous Occurrence of Potential Minima along Two Crystallographic Directions in an Octahedral Potential, Phys. Rev. B 34, 1277 (1986).

[84] A. S. Barker and A. J. Sievers, Optical Studies of the Vibrational Properties of Disordered Solids, Rev. Mod. Phys. 47, S1 (1975).

[85] Z. Leghtas, S. Touzard, I. M. Pop, A. Kou, B. Vlastakis, A. Petrenko, K. M. Sliwa, A. Narla, S. Shankar, M. J. Hatridge, M. Reagor, L. Frunzio, R. J. Schoelkopf, M. Mirrahimi, and M. H. Devoret, Confining the State of Light to a Quantum Manifold by Engineered Two-Photon Loss, Science 347 (2015).

[86] B. Shepperson, A. S. Chatterley, A. A. Sondergaard, L. Christiansen, M. Lemeshko, and H. Stapelfeldt, Strongly Aligned Molecules inside Helium Droplets in the NearAdiabatic Regime, J. Chem. Phys. 147, 013946 (2017).

[87] S. Ospelkaus, K.-K. Ni, G. Quéméner, B. Neyenhuis, D. Wang, M. H. G. de Miranda, J. L. Bohn, J. Ye, and D. S. Jin, Controlling the Hyperfine State of Rovibronic Ground-State Polar Molecules, Phys. Rev. Lett. 104, 030402 (2010).

[88] T. Unden, P. Balasubramanian, D. Louzon, Y. Vinkler, M. B. Plenio, M. Markham, D. Twitchen, A. Stacey, I. Lovchinsky, A. O. Sushkov, M. D. Lukin, A. Retzker, B. Naydenov, L. P. McGuinness, and F. Jelezko, Quantum Metrology Enhanced by Repetitive Quantum Error Correction, Phys. Rev. Lett. 116, 230502 (2016).

[89] D. K. L. Oi, V. Potocek, and J. Jeffers, Nondemolition Measurement of the Vacuum State or Its Complement, Phys. Rev. Lett. 110, 210504 (2013).

[90] J. C. J. Radtke, D. K. L. Oi, and J. Jeffers, Linear Quantum Optical Bare Raising Operator, J. Phys. B 50, 215501 (2017).

[91] J. A. Gross, Encoding a Qubit in a Spin, arXiv:2005.10910.

[92] M. M. Postnikov, Three-Dimensional Spherical Forms, in Discrete Geometry and Topology, Dedicated to the 100th Anniversary of the Birth of Boris Nikolaevich Delone, Proc. Steklov Inst. Math., Vol. 196, edited by E. F. Mishchenko and S. P. Novikov (Nauka, Moscow, 1992), 
p. 129, http://www.mathnet.ru/php/archive.phtml?wshow= paper\&jrnid=tm\&paperid=1458\&option_lang=eng.

[93] J. H. Conway and D. H. Huson, The Orbifold Notation for Two-Dimensional Groups, Structural chemistry 13, 247 (2002).

[94] J. M. Montesinos-Amilibia, Classical Tessellations and Three-Manifolds (Springer-Verlag, Berlin, 1987), https:// doi.org/10.1007/978-3-642-61572-6.

[95] C. Wulker, S. Ruan, and G. S. Chirikjian, Quantizing Euclidean Motions via Double-Coset Decomposition, Research 2019, 1608396 (2019).

[96] W. P. Thurston, in Three-Dimensional Geometry and Topology, edited by S. Levy (Princeton University, Princeton, NJ, 1997), https://press.princeton.edu/titles/6086.html.

[97] J. R. Weeks, The Shape of Space: How to Visualize Surfaces and Three-dimensional Manifolds (Marcel Dekker, New York, 1985), https://books.google.com/ books/about/The_Shape_of_Space.html? id $=$ mVHvAAAAMAAJ.

[98] F. T. Arecchi, E. Courtens, R. Gilmore, and H. Thomas, Atomic Coherent States in Quantum Optics, Phys. Rev. A 6, 2211 (1972).

[99] A. M. Perelomov, Generalized Coherent States and Their Applications (Springer, New York, 1986), https://doi.org/ 10.1007/978-3-642-61629-7.

[100] F. Haas, J. Volz, R. Gehr, J. Reichel, and J. Esteve, Entangled States of More Than 40 Atoms in an Optical Fiber Cavity, Science 344, 180 (2014).

[101] H. Strobel, W. Muessel, D. Linnemann, T. Zibold, D. B. Hume, L. Pezze, A. Smerzi, and M. K. Oberthaler, Fisher Information and Entanglement of Non-Gaussian Spin States, Science 345, 424 (2014).

[102] B. Lücke, J. Peise, G. Vitagliano, J. Arlt, L. Santos, G. Tóth, and C. Klempt, Detecting Multiparticle Entanglement of Dicke States, Phys. Rev. Lett. 112, 155304 (2014).

[103] R. McConnell, H. Zhang, J. Hu, S. Ćuk, and V. Vuletić, Entanglement with Negative Wigner Function of Almost 3,000 Atoms Heralded by One Photon, Nature (London) 519, 439 (2015).

[104] L. Pezzè, A. Smerzi, M. K. Oberthaler, R. Schmied, and P. Treutlein, Quantum Metrology with Nonclassical States of Atomic Ensembles, Rev. Mod. Phys. 90, 035005 (2018).

[105] S. Kobayashi, M. Kobayashi, Y. Kawaguchi, M. Nitta, and M. Ueda, Abe Homotopy Classification of Topological Excitations under the Topological Influence of Vortices, Nucl. Phys. B856, 577 (2012).

[106] D. M. Stamper-Kurn and M. Ueda, Spinor Bose Gases: Symmetries, Magnetism, and Quantum Dynamics, Rev. Mod. Phys. 85, 1191 (2013).

[107] R. Barnett, A. Turner, and E. Demler, Classifying Novel Phase of Spinor Atoms, Phys. Rev. Lett. 97, 180412 (2006).

[108] C.S. Jackson, How to Implement the GeneralizedCoherent-State POVM via Nonadaptive Continuous Isotropic Measurement, arXiv:1903.02045.

[109] D. A. Varshalovich, A. N. Moskalev, and V. K. Khersonskii, Quantum Theory of Angular Momentum (World Scientific, Singapore, 1988), https://doi.org/10.1142/0270.

[110] E. Brion, K. Molmer, and M. Saffman, Quantum Computing with Collective Ensembles of Multilevel Systems, Phys. Rev. Lett. 99, 260501 (2007).
[111] E. Brion, L. H. Pedersen, M. Saffman, and K. Molmer, Error Correction in Ensemble Registers for Quantum Repeaters and Quantum Computers, Phys. Rev. Lett. 100, 110506 (2008).

[112] M. Saffman and K. Molmer, Scaling the Neutral-Atom Rydberg Gate Quantum Computer by Collective Encoding in Holmium Atoms, Phys. Rev. A 78, 012336 (2008).

[113] S. H. Youn, M. Lu, U. Ray, and B. L. Lev, Dysprosium Magneto-Optical Traps, Phys. Rev. A 82, 043425 (2010).

[114] J. Miao, J. Hostetter, G. Stratis, and M. Saffman, MagnetoOptical Trapping of Holmium Atoms, Phys. Rev. A 89, 041401(R) (2014).

[115] J. J. McClelland and J. L. Hanssen, Laser Cooling without Repumping: A Magneto-Optical Trap for Erbium Atoms, Phys. Rev. Lett. 96, 143005 (2006).

[116] M. Lu, N. Q. Burdick, S. H. Youn, and B. L. Lev, Strongly Dipolar Bose-Einstein Condensate of Dysprosium, Phys. Rev. Lett. 107, 190401 (2011).

[117] M. Lu, N. Q. Burdick, and B. L. Lev, Quantum Degenerate Dipolar Fermi Gas, Phys. Rev. Lett. 108, 215301 (2012).

[118] K. Aikawa, A. Frisch, M. Mark, S. Baier, A. Rietzler, R. Grimm, and F. Ferlaino, Bose-Einstein Condensation of Erbium, Phys. Rev. Lett. 108, 210401 (2012).

[119] K. Aikawa, A. Frisch, M. Mark, S. Baier, R. Grimm, and F. Ferlaino, Reaching Fermi Degeneracy via Universal Dipolar Scattering, Phys. Rev. Lett. 112, 010404 (2014).

[120] E. Urban, N. Glikin, S. Mouradian, K. Krimmel, B. Hemmerling, and H. Haeffner, Coherent Control of the Rotational Degree of Freedom of a Two-Ion Coulomb Crystal, Phys. Rev. Lett. 123, 133202 (2019).

[121] S. M. Girvin, Circuit QED: Superconducting Qubits Coupled to Microwave Photons, in Quantum Machines: Measurement and Control of Engineered Quantum Systems, edited by M. H. Devoret, B. Huard, R. J. Schoelkopf, and L.F. Cugliandolo (Oxford University Press, New York, 2015), Chap. 3, https://doi.org/ 10.1093/acprof:oso/9780199681181.001.0001.

[122] A. Nicolas, L. Veissier, L. Giner, E. Giacobino, D. Maxein, and J. Laurat, A Quantum Memory for Orbital Angular Momentum Photonic Qubits, Nat. Photonics 8, 234 (2014).

[123] A. L. Grimsmo, J. Combes, and B. Q. Baragiola, Quantum Computing with Rotation-Symmetric Bosonic Codes, Phys. Rev. X 10, 011058 (2020).

[124] M. P. Fisher, Quantum Cognition: The Possibility of Processing with Nuclear Spins in the Brain, Ann. Phys. (Amsterdam) 362, 593 (2015).

[125] N. Y. Halpern and E. Crosson, Quantum Information in the Posner Model of Quantum Cognition, Ann. Phys. (Amsterdam) 407, 92 (2019).

[126] J.-P. Luminet, J. R. Weeks, A. Riazuelo, R. Lehoucq, and J.-P. Uzan, Dodecahedral Space Topology as an Explanation for Weak Wide-Angle Temperature Correlations in the Cosmic Microwave Background, Nature (London) 425, 593 (2003).

[127] D. Jüstel, The Zak Transform on Strongly Proper G-Spaces and Its Applications, J. Lond. Math. Soc. 97, 47 (2018).

[128] M. Bander and C. Itzykson, Group Theory and the Hydrogen Atom (I), Rev. Mod. Phys. 38, 330 (1966). 
[129] L. Childress, M. P. Schmidt, A. D. Kashkanova, C. D. Brown, G. I. Harris, A. Aiello, F. Marquardt, and J. G. E. Harris, Cavity Optomechanics in a Levitated Helium Drop, Phys. Rev. A 96, 063842 (2017).

[130] Y. Arita, M. Mazilu, and K. Dholakia, Laser-Induced Rotation and Cooling of a Trapped Microgyroscope in Vacuum, Nat. Commun. 4, 2374 (2013).

[131] T. M. Hoang, Y. Ma, J. Ahn, J. Bang, F. Robicheaux, Z.-Q. Yin, and T. Li, Torsional Optomechanics of a Levitated Nonspherical Nanoparticle, Phys. Rev. Lett. 117, 123604 (2016).

[132] L. P. Neukirch, E. von Haartman, J. M. Rosenholm, and A. N. Vamivakas, Multi-dimensional Single-Spin NanoOptomechanics with a Levitated Nanodiamond, Nat. Photonics 9, 653 (2015).

[133] R. Reimann, M. Doderer, E. Hebestreit, R. Diehl, M. Frimmer, D. Windey, F. Tebbenjohanns, and L. Novotny, $\mathrm{GHz}$ Rotation of an Optically Trapped Nanoparticle in Vacuum, Phys. Rev. Lett. 121, 033602 (2018).

[134] J. Ahn, Z. Xu, J. Bang, Y.-H. Deng, T. M. Hoang, Q. Han, R.-M. Ma, and T. Li, Optically Levitated Nanodumbbell Torsion Balance and $\mathrm{GHz}$ Nanomechanical Rotor, Phys. Rev. Lett. 121, 033603 (2018).

[135] U. Delic, M. Reisenbauer, D. Grass, N. Kiesel, V. Vuletic, and M. Aspelmeyer, Cavity Cooling of a Levitated Nanosphere by Coherent Scattering, Phys. Rev. Lett. 122, 123602 (2019).

[136] B. A. Stickler, B. Papendell, S. Kuhn, B. Schrinski, J. Millen, M. Arndt, and K. Hornberger, Probing Macroscopic Quantum Superpositions with Nanorotors, New J. Phys. 20, 122001 (2018).

[137] J. S. Pedernales, F. Cosco, and M. B. Plenio, DecoherenceFree Rotational Degrees of Freedom for Quantum Applications, arXiv:2001.00826.

[138] D. Gottesman, Stabilizer Codes and Quantum Error Correction, Ph.D. Thesis, California Institute of Technology, 1997, https://arxiv.org/abs/quant-ph/9705052.

[139] J. Preskill, Lecture Notes on Quantum Computation (online notes), http://www.theory.caltech.edu/ preskill/ ph219/index.html\#lecture.

[140] P. Raynal, A. Kalev, J. Suzuki, and B.-G. Englert, Encoding Many Qubits in a Rotor, Phys. Rev. A 81, 052327 (2010).

[141] M. Grassl, in Lectures on Quantum Information, edited by D. Bruß and G. Leuchs (Wiley-VCH, New York, 2006), https://www.wiley.com/en-gb/Lectures+on+Quantum +Information-p-9783527405275.

[142] J. Bermejo-Vega, C. Y.-Y. Lin, and M. Van den Nest, Normalizer Circuits and a Gottesman-Knill Theorem for Infinite-Dimensional Systems, Quantum Inf. Comput. 16, 361 (2016), http://www.rintonpress.com/journals/ qiconline.html\#v16n910.

[143] J. Bermejo-Vega, Normalizer Circuits and Quantum Computation, Ph.D. thesis, Technical University of Munich, 2016, arXiv:1611.09274.

[144] A. M. Steane, Active Stabilization, Quantum Computation, and Quantum State Synthesis, Phys. Rev. Lett. 78, 2252 (1997).

[145] We are free to choose any orthonormal basis we like for the irrep $D^{\ell}$. When an explicit form for $D_{m n}^{\ell}$ is needed, we use the complex-conjugated Wigner $D$ functions from Sec. IV. 3 in Ref. [109].

[146] S. Ramakrishna and T. Seideman, Intense Laser Alignment in Dissipative Media as a Route to Solvent Dynamics, Phys. Rev. Lett. 95, 113001 (2005).

[147] C. Zhong and F. Robicheaux, Decoherence of Rotational Degrees of Freedom, Phys. Rev. A 94, 052109 (2016).

[148] B. A. Stickler, B. Papendell, and K. Hornberger, SpatioOrientational Decoherence of Nanoparticles, Phys. Rev. A 94, 033828 (2016).

[149] R. Schmidt and M. Lemeshko, Rotation of Quantum Impurities in the Presence of a Many-Body Environment, Phys. Rev. Lett. 114, 203001 (2015).

[150] B. Papendell, B. A. Stickler, and K. Hornberger, Quantum Angular Momentum Diffusion of Rigid Bodies, New J. Phys. 19, 122001 (2017).

[151] B. A. Stickler, B. Schrinski, and K. Hornberger, Rotational Friction and Diffusion of Quantum Rotors, Phys. Rev. Lett. 121, 040401 (2018).

[152] B. A. Stickler, F. T. Ghahramani, and K. Hornberger, Rotational Alignment Decay and Decoherence of Molecular Superrotors, Phys. Rev. Lett. 121, 243402 (2018).

[153] D. P. Arovas, Lecture Notes on Group Theory in Physics (online notes), https://courses.physics.ucsd.edu/2016/ Spring/physics220/LECTURES/GROUP_THEORY.pdf.

[154] G. S. Chirikjian and A. B. Kyatkin, Engineering Applications of Noncommutative Harmonic Analysis: With Emphasis on Rotation and Motion Groups (CRC Press, Boca Rato, FL, 2000), https://www.amazon.com/EngineeringApplications-Noncommutative-Harmonic-Analysis/dp/ 0849307481.

[155] J. Preskill, Gauge Anomalies in an Effective Field Theory, Ann. Phys. (N.Y.) 210, 323 (1991).

[156] H. Bacry, Group Theory and Constellations (Editions Publibook, 2004), http://www.publibook.com/doc/25797/.

[157] P. Leboeuf and A. Voros, Chaos-Revealing Multiplicative Representation of Quantum Eigenstates, J. Phys. A 23, 1765 (1990).

[158] R. Schmidt and M. Lemeshko, Deformation of a Quantum Many-Particle System by a Rotating Impurity, Phys. Rev. X 6, 011012 (2016).

[159] M. Fallbacher, Breaking Classical Lie Groups to Finite Subgroups-An Automated Approach, Nucl. Phys. B898, 229 (2015).

[160] C. H. Bennett, D. P. DiVincenzo, J. A. Smolin, and W. K. Wootters, Mixed-State Entanglement and Quantum Error Correction, Phys. Rev. A 54, 3824 (1996).

[161] E. Knill and R. Laflamme, Theory of Quantum ErrorCorrecting Codes, Phys. Rev. A 55, 900 (1997).

[162] M. A. Nielsen and I. L. Chuang, Quantum Computation and Quantum Information (Cambridge University Press, Cambridge, England, 2011), https://doi.org/10.1017/ CBO9780511976667.

[163] P. Aliferis and J. Preskill, Fault-Tolerant Quantum Computation against Biased Noise, Phys. Rev. A 78, 052331 (2008).

[164] D. W. Leung, M. A. Nielsen, I. L. Chuang, and Y. Yamamoto, Approximate Quantum Error Correction Can Lead to Better Codes, Phys. Rev. A 56, 2567 (1997). 
[165] C. Crépeau, D. Gottesman, and A. Smith, Approximate Quantum Error-Correcting Codes and Secret Sharing Schemes, in Adv. Cryptol.-EUROCRYPT 2005, Lecture Notes in Computer Science Vol. 3494, edited by R. Cramer (Springer, Berlin, 2005), pp. 285-301, https://doi.org/ 10.1007/11426639_17.

[166] M. Lachièze-Rey, A New Basis for Eigenmodes on the Sphere, J. Phys. A 37, 205 (2004).

[167] T. O. Sherman, Fourier Analysis on the Sphere, Trans. Am. Math. Soc. 209, 1 (1975).

[168] I. P. Volobuev, Plane Waves on a Sphere and Some Applications, Theor. Math. Phys. 45, 1119 (1980).

[169] G. Nebe, E. M. Rains, and N. J. A. Sloane, Self-Dual Codes and Invariant Theory, Algorithms and Computation in Mathematics Vol. 17 (Springer-Verlag, Berlin, 2006), https://doi.org/10.1007/3-540-30731-1.

[170] R. H. Hardin and N. J. A. Sloane, McLaren's Improved Snub Cube and Other New Spherical Designs in Three Dimensions, Discrete Comput. Geom. 15, 429 (1996).

[171] F. Lacerda, J. M. Renes, and V. B. Scholz, Coherent State Constellations for Bosonic Gaussian Channels, in Proceedings of the 2016 IEEE International Symposium on Information Theory (IEEE, New York, 2016), p. 2499, https://doi.org/10.1109/ISIT.2016.7541749.

[172] V. V. Albert, S. Pascazio, and M. H. Devoret, General Phase Spaces: From Discrete Variables to Rotor and Continuum Limits, J. Phys. A 50, 504002 (2017).

[173] B. C. Hall, Quantum Theory for Mathematicians, Graduate Texts in Mathematics Vol. 267 (Springer, New York, 2013), https://doi.org/10.1007/978-1-4614-7116-5.

[174] A. Barut and R. Raczka, Theory of Group Representations and Applications (World Scientific, Singapore, 1986), https://doi.org/10.1142/0352.

[175] C. G. Brell, Generalized Cluster States Based on Finite Groups, New J. Phys. 17, 023029 (2015).

[176] J. Kogut and L. Susskind, Hamiltonian Formulation of Wilson's Lattice Gauge Theories, Phys. Rev. D 11, 395 (1975).

[177] E. Zohar and M. Burrello, Formulation of Lattice Gauge Theories for Quantum Simulations, Phys. Rev. D 91, 054506 (2015).

[178] To show the above, we use $g=a h$ with $a \in \mathrm{F}_{\mathrm{G} / \mathrm{H}}$ and $h \in \mathrm{H}$ for each $g \in \mathrm{G},\left\langle a h \mid a^{\prime} h^{\prime}\right\rangle=\delta_{a a^{\prime}}^{\mathrm{G} / \mathrm{H}} \delta_{h h^{\prime}}^{\mathrm{H}}$, and Table IV E. We define generalized delta functions $\delta_{x y}^{\mathrm{X}}$ for a space X, satisfying $(1 /|\mathrm{X}|) \sum_{y \in \mathrm{X}} f(y) \delta_{x y}^{\mathrm{X}}=f(x)$ for $x \in \mathrm{X}$.

[179] A. M. Childs and W. van Dam, Quantum Algorithms for Algebraic Problems, Rev. Mod. Phys. 82, 1 (2010).

[180] P. Faist, S. Nezami, V. V. Albert, G. Salton, F. Pastawski, P. Hayden, and J. Preskill, Continuous Symmetries and Approximate Quantum Error Correction, arXiv:1902.07714.
[181] K. Noh, S. M. Girvin, and L. Jiang, Encoding an Oscillator into Many Oscillators, Phys. Rev. Lett. 125, 080503 (2020).

[182] F. Iachello, F. Pérez-Bernal, and P. Vaccaro, A Novel Algebraic Scheme for Describing Nonrigid Molecules, Chem. Phys. Lett. 375, 309 (2003).

[183] H. Schmiedt, P. Jensen, and S. Schlemmer, Collective Molecular Superrotation: A Model for Extremely Flexible Molecules Applied to Protonated Methane, Phys. Rev. Lett. 117, 223002 (2016).

[184] S. Buzaglo, E. Yaakobi, T. Etzion, and J. Bruck, Systematic Error-Correcting Codes for Permutations and Multi-Permutations, IEEE Trans. Inf. Theory 62, 3113 (2016).

[185] S. Stellmer, F. Schreck, and T. C. Killian, Degenerate Quantum Gases of Strontium, in Annual Review of Cold Atoms and Molecules (World Scientific, Singapore, 2014), Chap. 1, pp. 1-80, https://doi.org/10.1142/ 9789814590174_0001.

[186] M. T. Vaughn, Introduction to Mathematical Physics (Wiley, Weinheim, 2007), https://doi.org/10.1002/ 9783527618859.

[187] R. W. Carter, I. G. MacDonald, and G. B. Segal, Lectures on Lie Groups and Lie Algebras (Cambridge University Press, Cambridge, England, 1995), https://doi.org/ 10.1017/CBO9781139172882.

[188] A. G. Farashahi, Peter-Weyl Theorem for Homogeneous Spaces of Compact Groups, Int. J. Anal. Appl. 13, 22 (2017), http://etamaths.com/index.php/ijaa/article/view/792.

[189] N. J. Vilenkin and A. U. Klimyk, Representation of Lie Groups and Special Functions (Springer Netherlands, Dordrecht, 1991), https://doi.org/10.1007/978-94-0113538-2.

[190] M. Tinkham, Group Theory and Quantum Mechanics (Dover, New York, 1964), https://www.amazon.com/ Group-Theory-Quantum-Mechanics-Chemistry/dp/ 0486432475.

[191] G. B. Folland, Harmonic Analysis in Phase Space (Princeton University, Princeton, NJ, 1989), https://press .princeton.edu/titles/4398.html.

[192] J. Zak, Finite Translations in Solid-State Physics, Phys. Rev. Lett. 19, 1385 (1967).

[193] H. G. Feichtinger and T. Strohmer, Introduction, in Gabor Analysis and Algorithms, edited by H. G. Feichtinger and T. Strohmer (Birkhäuser, Basel, 1998), Chap. 1, https:// doi.org/10.1007/978-1-4612-2016-9.

[194] Y. Aharonov, H. Pendleton, and A. Petersen, Modular Variables in Quantum Theory, Int. J. Theor. Phys. 2, 213 (1969).

[195] D. Marinucci and G. Peccati, Random Fields on the Sphere (Cambridge University Press, Cambridge, England, 2011), https://doi.org/10.1017/CBO9780511751677. 